\begin{abstract}
WARD, JON ROBERT. Chip Discrimination for UWB Impulse Radio

Networks in Multipath Channels (Under the direction of Professor J. Keith Townsend).

The recent need for increasingly higher data rates and the ability to utilize unused spectrum has fueled growing interest in UWB from both academia and industry. A variety of UWB physical layer applications have been proposed and implemented including: highspeed, short-range indoor wireless personal area networks (WPAN's), product sensor tags, and low-power, covert military networks. Much of the UWB literature focuses on the Impulse Radio (IR) variety which is characterized by the transmission of multiple timedithered, short duration pulses per data bit. Researchers have consistently studied IR by making key assumptions to simplify system analysis, namely perfect multi-user power control and single path of signal arrival.
\end{abstract}

In the military networks of interest, a variety of line-of-sight (LOS) and non line-ofsight (NLOS) paths exist between transceivers operating at different power levels. Careful power control becomes complex when no central node provides service to the entire network and a small number of high-powered co-located users can quickly degenerate system performance. We use computer simulation to investigate the system degradation incurred, in terms of Bit Error Rate (BER), in a multi-user IR network with large near-far power disparity operating in a multi-user indoor environment. We then extend the work presented in [1] for the multi-user Gaussian channel as a solution to mitigate the effects of high-powered interferer signals in the IEEE 802.15.3a indoor multipath environment.

Three varieties of RAKE receivers commonly found in literature are investigated: ARAKE, S-RAKE, and P-RAKE. We introduce a novel chip discriminator located at each RAKE finger output that selectively removes finger demodulation metrics based on the product of a constant threshold level $T_{i n}$ and the estimated channel tap coefficient $\alpha_{k}$. A multi-user environment is considered where the desired user's signals pass through the NLOS channel CM2 and the co-located user's transmit through the LOS channel CM1. We quantify the performance of a system employing chip discrimination as well as its 
sensitivities to system parameters under the assumption of perfect channel estimation and maximal ratio combining (MRC). 


\title{
Chip Discrimination for UWB Impulse Radio Networks in Multipath Channels
}

\author{
by
}

\section{Jon R. Ward}

A thesis submitted to the Graduate Faculty of

North Carolina State University

in partial satisfaction of the

requirements for the Degree of

Master of Science

\section{Department of Electrical and Computer Engineering}

Raleigh

August 2005

Approved By:

Dr. Huaiyu Dai

Dr. J. Keith Townsend

Dr. Brian L. Hughes

Chair of Advisory Committee 
To Leigh and my family. 


\section{Biography}

Jon Robert Ward was born on January 10, 1981 in Greensboro, NC, the oldest child of Bruce and Sandra Ward. He graduated from Ragsdale High School in June, 1999. He received a B.S. degree in Computer and Electrical Engineering from North Carolina State University in May, 2003. He received his M.S. degree in Electrical Engineering in August 2005. After graduation he will join the Communication Systems Network Engineering Group (VIC) in the Power Projection Systems Department at the Johns Hopkins Applied Physics Laboratory. 


\section{Acknowledgements}

As one of a limited number of M.S. thesis students in the Electrical Engineering department that proceeded to graduate school after receiving a B.S. degree from North Carolina State University, I had the opportunity to build a relationship with each of my committee members. I would like to personally thank each of them beginning with my advisor and committee chair Dr. Keith Townsend. He had the patience to accept a student uninformed about the procedures of academic research and guide him to produce the work included in this thesis. I thank him for suggesting an interesting topic that relied heavily on the fundamentals of system simulation that can be used to solve a variety of communications problems. Dr. Townsend made himself available for advice and encouragement in what was seemingly one of the busiest times in his career and for that I am indebted.

I thank my committee members for their guidance in coursework that led me to pursue academic research. Dr. Brian Hughes first piqued my interest in communications systems as an undergraduate in ECE 402. He helped me begin my graduate studies at NCSU by serving as my interim advisor. I had the opportunity to study random processes with Dr. Huaiyu Dai in one of the first classes he taught at NCSU. He is one the most approachable professors in the department and I was able to watch his teaching style develop in only a short time. I consider the opportunity to study under Drs. Hughes and Dai to have been a pleasure. I would also like to recognize Dr. Robert Ulman of the Army Research Office (ARO) and ARO contracts DAAD19-00-1-004 and DAAG55-98-D-0003, whose support made this project possible.

I have been blessed with a supportive family and I would like to express my appreciation for them and their part in making this thesis a reality. First, I would like to thank Leigh for her part in cooking, cleaning, and in general listening to my problems and complaints. Her encouragement as well as that of my family helped me to continue attacking the problem every day. I personally thank my parents Bruce and Sandra Ward and my grandparents Elsie and Carlton Ward for instilling in me the diligence that I not only applied to this project, but to the different situations that I encounter in life. 


\section{Contents}

List of Figures vii

List of Tables $\quad$ ix

1 Introduction $\quad 1$

1.1 Motivation ..................... 1

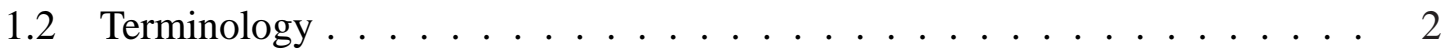

1.3 System Analysis Method \& Organization . . . . . . . . . . . . . . 3

2 Impulse Radio Physical Layer 6

2.1 Binary Pulse Position Modulation _. . . . . . . . . . . . . . . . . 8

2.2 Autonomous Chip Discrimination Method . . . . . . . . . . . . . . . 11

3 The Multipath Indoor UWB Channel $\quad 16$

3.1 Saleh-Valenzuela Channel Model . . . . . . . . . . . . . . . . . . . . . 17

3.2 IEEE 802.15.3a Modified S-V Channel Model . . . . . . . . . . . . . . . . 19

3.2.1 Modifications to the S-V Model . . . . . . . . . . . . . . 20

3.2.2 Discrete-Time Impulse Response . . . . . . . . . . . . . . . . . . 24

3.2.3 IEEE 802.15.3a Channel Model Limitations . . . . . . . . . . . . . 27

3.2 .4 Specific Modifications . . . . . . . . . . . . . . . 28

3.2.5 Multipath Characteristics of the IEEE 802.15.3a Channel . . . . . . 30

3.3 The RAKE Receiver . . . . . . . . . . . . . . . . . . . 33

3.3.1 RAKE Receiver Varieties . . . . . . . . . . . . . . . . . 35

4 Simulation Model $\quad 37$

4.1 Model Environment . . . . . . . . . . . . . . . . . . . . . . . 37

4.2 System Model . . . . . . . . . . . . . . . . . . . . . . . . . 39

4.2 .1 Assumptions . . . . . . . . . . . . . . . . . 40

4.2.2 Simulation Parameters . . . . . . . . . . . . . . 42 
5 Multi-User Multipath System Model with Chip Discrimination 44

5.1 Received Signal . . . . . . . . . . . . . . . . . . . . . 45

5.2 Chip Discrimination . . . . . . . . . . . . . . . . . . 45

6 Results 50

6.1 Threshold Performance . . . . . . . . . . . . . . . . . . 51

6.2 System Performance Sensitivity . . . . . . . . . . . . . . . 56

6.3 Reliability of Discarding Chips . . . . . . . . . . . . . . . . 62

6.4 Imperfect Channel Estimation . . . . . . . . . . . . . . . . . . . . 66

7 Conclusions and Future Work $\quad 72$

$\begin{array}{ll}\text { Bibliography } & 77\end{array}$ 


\section{List of Figures}

2.1 Ideal transmitted and received pulse shapes . . . . . . . . . . . . . 7

2.2 BPPM modulated waveforms . . . . . . . . . . . . . . . . 10

2.3 BPPM correlation receiver template waveform . . . . . . . . . . . . . . 11

2.4 BPPM data link and physical layer example . . . . . . . . . . . . . . 12

2.5 System block diagram of chip discrimination in the Gaussian Channel . . . 14

3.1 Decaying Power Delay Profile (PDP) demonstrating clusters \& rays . . . . 18

3.2 CM1 example continuous-time \& discrete-time impulse responses . . . . . 30

3.3 CM2 example continuous-time \& discrete-time impulse responses . . . . . 31

3.4 Maximal Ratio Combining (MRC) RAKE receiver . . . . . . . . . . . . . 34

4.1 Diagram of multi-user simulation environment . . . . . . . . . . . . . . 38

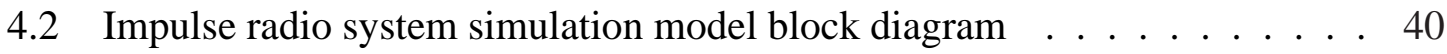

5.1 Maximal Ratio Combining (MRC) RAKE receiver with chip discrimination 47

5.2 Multipath Component (MPC) chip discriminator diagram . . . . . . . . . 47

6.1 Average BER achieved for varying chip discriminator threshold $T_{i n} \ldots$. . . 52

6.2 Average BER achieved for varying $N_{s}$ with no chip discrimination . . . . . 54

6.3 A-RAKE 3d plot of average BER for varying $T_{\text {in }}$ threshold and I/S levels . . 57

6.4 BER performance at threshold $T_{i n}=-56 \mathrm{~dB}$ for varying I/S . . . . . . . . . 58

6.5 Average BER versus $N_{s}$ at optimal BER threshold $T_{i n} \ldots \ldots$. . . . . . . 60

6.6 Average BER versus $N_{\text {Near }}$ at optimal BER threshold $T_{\text {in }} \ldots \ldots$. . . . . . 61

6.7 Probability of discarding chips at varying threshold $T_{\text {in }}$ and co-located interferer density $N_{\text {Near }}$ for A-RAKE, S-RAKE, and P-RAKE . . . . . . . . 63

6.8 Probability of discarding chips at varying threshold $T_{\text {in }}$ and co-located interferer density $N_{\text {Near }}$ for A-RAKE . . . . . . . . . . . . . . . . 64

6.9 Probability of discarding chips at varying threshold $T_{i n}$ and co-located interferer power disparity $(I / S)_{T X}$ for A-RAKE . . . . . . . . . . . 66

6.10 Average BER versus threshold $T_{\text {in }}$ for A-RAKE with varying $S N R_{\text {err }} \ldots$. . . 68

6.11 Average BER versus threshold $T_{i n}$ for S-RAKE with varying $S N R_{\text {err }} \ldots$. . . 69 
6.12 Average BER versus threshold $T_{\text {in }}$ for P-RAKE with varying $S N R_{\text {err }} \ldots \ldots$ 


\section{List of Tables}

3.1 UWB channel model large-scale path loss attenuation and exponent factors 24

3.2 IEEE 802.15.3a UWB channel model parameter description . . . . . . . . 25

3.3 IEEE 802.15.3a UWB channel model parameter values . . . . . . . . . . . 26

3.4 IEEE 802.15.3a discrete-time channel model parameter description . . . . . 27

3.5 Statistical multipath characterization parameters for Tx-Rx separation $1 \mathrm{~m}$. 32

3.6 Statistical multipath characterization parameters for Tx-Rx separation $2 \mathrm{~m}$. 33

4.1 System simulation model parameters . . . . . . . . . . . . . . . . . 43 


\section{Chapter 1}

\section{Introduction}

\subsection{Motivation}

The UWB physical layer is a viable solution to wireless military communications networks as the inherent properties of impulse radio (IR) can satisfy many of the design criteria: low probability of detection and interception (LPD and LPI), high system throughput, and low transmitter power levels. An equally important design criterion of a wireless military network is survivability; there should be no single node with total network control or a node that damages the network beyond repair when lost. This system constraint favors an ad hoc network structure.

The cellular network topology provides centralized power control between nodes that is not available in an ad hoc network. Power control in a multi-user system is of primary concern in terms of system performance; if large near-far power ratios exist in the network, an interfering signal can overwhelm the desired received signal and force a bit error. Each node could transmit power control information in packet headers, but this requires system cooperation and increased complexity while decreasing system throughput. An autonomous solution to mitigate the performance loss of a multi-user ad hoc network with 
large near-far power ratios was proposed in [1]. The aforementioned work presents a passive technique called "chip discrimination" that exploits the IR spreading sequence $N_{s}$ and applies an acceptance level threshold to each demodulated voltage metric.

The chip-discrimination technique presented in [1] was applied to the Gaussian channel where the desired and multi-user signals randomly arrive at the receiver via a single path corrupted only by multi-user interference and thermal noise. Although this development provides a theoretical framework, it does not account for the multipath present in all wireless networks. UWB is unique in that the narrow received pulses allow very fine resolution of multipath components (MPCs) at the receiver. We extend the chip-discrimination technique presented in [1] as a solution to mitigate the system performance loss due to strong interferer signals in the indoor multipath environment.

\subsection{Terminology}

We attempt to avoid ambiguity in the specific terms commonly found throughout this thesis by listing and defining the most frequently found terminology:

- Multipath Component (MPC) - A single received pulse at the channel output belonging to the set of $L$ attenuated, delayed, and distorted transmitted pulses that compose the channel output given by Equation 5.1. Each MPC is individually tracked and demodulated by a single RAKE receiver finger.

- Chip - One of the $N_{s}$ pulses transmitted for each data bit. When discussing chip discrimination, we often refer to the resolved MPCs as chips because $L$ MPCs are produced by the channel from a single transmitted chip.

- Chip Discrimination - The application of a chip discriminator device to the demodulator output that compares the energy collected from a demodulated received chip to a threshold. If the demodulation metric exceeds the threshold, it is discarded; otherwise, the received chip contributes to the bit estimate. No distinction is made 
between applying the technique to a single arriving chip as in the Gaussian channel or many MPCs as in the multipath channel.

- $Z_{k}$ - The $k^{\text {th }}$ RAKE receiver demodulated finger output as shown in Fig. 3.4.

- Maximal Ratio Combining (MRC) - A linear combining technique that scales the $k^{\text {th }}$ RAKE receiver demodulated finger output $Z_{k}$ by the $k^{\text {th }}$ channel tap coefficient $\alpha_{k}$ before summing over all $L$ resolvable components [2].

- A-RAKE (All) Receiver - The ideal RAKE receiver with unlimited resources to track and demodulate all $L$ resolvable MPCs.

- S-RAKE (Selective) Receiver - A RAKE receiver variety that resolves the $L_{s}$ MPCs resulting from paths with the largest $L_{s}$ tap weights $\alpha_{k}$, where $L_{s} \ll L$.

- P-RAKE (Partial) Receiver - A RAKE receiver variety that resolves the first arriving $L_{p}$ MPCs, where $L_{p} \ll L$.

\subsection{System Analysis Method \& Organization}

We develop a multi-user ad hoc network environment with large near-far ratio such that the transmit power of co-located interferers is much larger than that of the desired user. Other signal-degrading contributions such as background user interference and ther-

mal noise at the receiver are modeled as a zero-mean Gaussian process with variance $\sigma_{b g}^{2}$ chosen to maintain a constant average background SNR $\overline{S N R_{b g}}$. We model multipath fading using the IEEE 802.15.3a indoor channel model at each network node based on the multipath channel statistical parameters; the desired user's signals pass through the NLOS channel CM2 and the co-located user's transmit through the LOS channel CM1. We choose to investigate system performance using the three most common RAKE receiver varieties found in literature: A-RAKE (ALL-RAKE), S-RAKE (Selective-RAKE), and P-RAKE (Partial-RAKE). 
We introduce a novel chip discriminator device located at each RAKE finger output that selectively removes finger demodulation metrics based on the product of a constant threshold level $T_{\text {in }}$ and the estimated channel tap coefficient $\alpha_{k}$. Each resolved multipath component (MPC) that exceeds the chip discriminator threshold is discarded from the bit decision process. Otherwise a large demodulated RAKE finger metric continues to corrupt the estimated metric for an entire bit duration. Simulation results are compared to quantify the performance gain of a multi-user system incorporating chip discrimination in a multipath environment over a multi-user system that employs no power control technique. The sensitivity of the system employing chip discrimination to varying system parameters is also investigated under the assumption of perfect channel estimation and maximal ratio combining (MRC).

The organization of this thesis is as follows. Chapter 2 presents the UWB impulse radio physical layer based on the work reported in $[3,4,5]$. This section gives the basic background of an IR system assuming that the reader has some prior knowledge of the subject. Section 2.2 summarizes the chip-discrimination technique for the Gaussian channel as reported in [1]. All of the details needed to extend the basic chip discrimination technique to a more realistic indoor multipath environment are presented in this chapter.

Chapter 3 gives a detailed description of the recently accepted IEEE 802.15.3a indoor UWB channel model. We present the evolution of the channel model from its first development and publication in [6] to its current adaptation in $[7,8,9]$ based on statistical multipath characterization parameters evaluated from indoor data measurements. We then develop the discrete-time impulse response and discuss model limitations. The specific channel model modifications used to arrive at the analyzed channel model are given along with the evaluation of statistical channel parameters based on this model. The last section includes a discussion on the generic RAKE receiver as well as three varieties commonly found in literature: A-RAKE, S-RAKE, and P-RAKE. This section is intended to concisely present the IEEE 802.15.3a channel model such that it can easily be implemented after analyzing Chapter 3. 
We present the underlying simulation model in Chapter 4 including an illustration of the network environment in Section 4.1. We clearly state the system assumptions used to produce all performance results in Section 4.2.1 followed by the specific simulation parameters in Section 4.2.2. Chapter 5 presents the details of the chip-discrimination technique as applied to a multipath channel. This includes the mathematical representation of the received multi-user signal in Section 5.1 and the chip discriminator device in Section 5.2. A novel approach to chip discrimination is developed based on the work in [1] where the RAKE finger demodulation metrics $Z_{k}$ are compared to a constant threshold $T_{i n}$ and the estimated channel coefficient $\alpha_{k}$.

Chapter 6 presents system performance results for different levels of the constant threshold $T_{\text {in }}$ in Section 6.1. System sensitivity is examined by varying basic system parameters over a typical range to determine the effects on the system Bit Error Rate (BER) performance and the optimal BER constant threshold $T_{i n}$. The reliability of the system to discard RAKE finger outputs is analyzed for varying multi-user densities and co-located interferer energy levels in Section 6.3. The system BER performance for an impulse radio system employing chip discrimination is also investigated for imperfect channel tap coefficients $\tilde{\alpha_{k}}$ determined using a technique presented in [7]. We conclude in Chapter 7 by reiterating the usefulness of this work as well as related future work to improve applicability of the chip-discrimination technique. 


\section{Chapter 2}

\section{Impulse Radio Physical Layer}

The most fundamental requirement of an impulse radio system is to transmit low-power pulses such that the power spectral density (PSD) is as white as possible with energy spread evenly over the desired spectrum. The transmitted waveform $p(t)$ is the Gaussian monocycle first derivative which has a spectrum that is approximately flat over the bandwidth of the pulse and can be received by correlation detection virtually at the antenna terminals of the receiver [3]. We are most interested in system analysis at the receiver, where the received pulse waveform $w(t)$ is given mathematically in [10] by

$$
w(t)=\left[1-4 \pi\left\{\left(t-T_{p} / 2\right) /\left(0.39 T_{p}\right)\right\}^{2}\right] \exp \left[(-2 \pi)\left\{\left(t-T_{p} / 2\right) /\left(0.39 T_{p}\right)\right\}^{2}\right] .
$$

The waveform $w(t)$ is the second derivative Gaussian waveform after passing through the transmitter and receiver antennas where an approximate derivative occurs [4]. An example of the ideal transmitted and received pulses is shown in Figs. 2.1(a) and 2.1(b) respectively where both waveforms are normalized by the maximum voltage peak. Throughout this work we assume for simplicity that the transmitted and received waveforms $p(t)$ and $w(t)$ respectively are normalized to have unit energy.

Impulse radio systems transmit $N_{s}$ Gaussian monocycle pulses called chips for each 


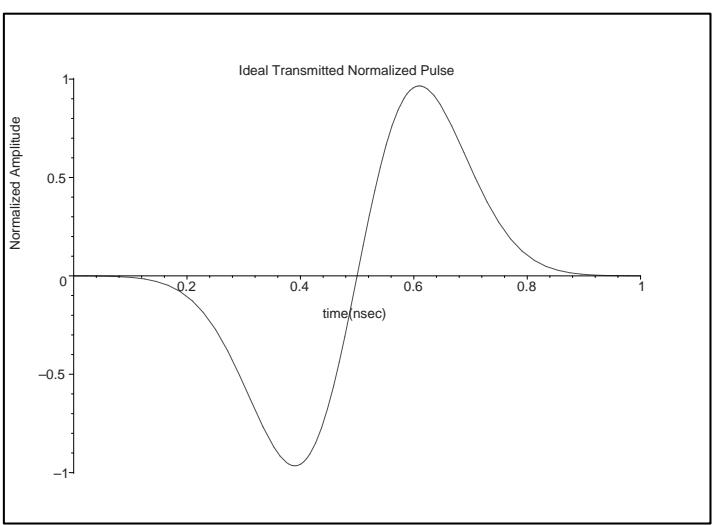

(a)

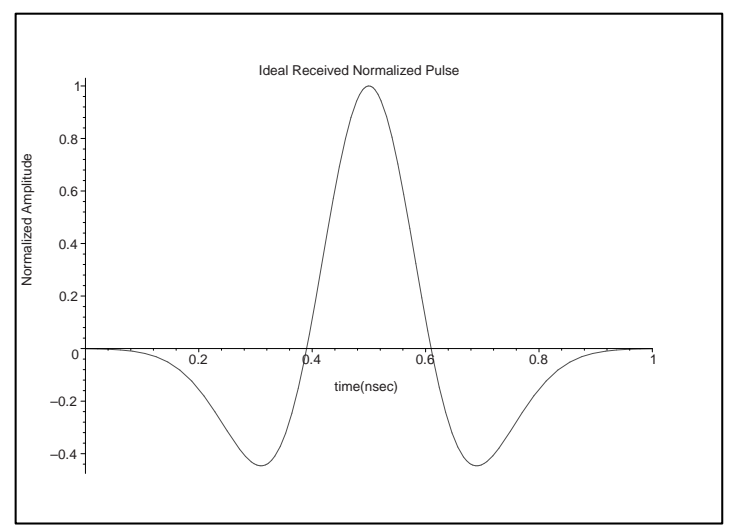

(b)

Figure 2.1: Fig. 2.1(a) is an amplitude normalized 1st derivative Gaussian waveform $p(t)$ representing one ideal transmitted chip. Fig. 2.1(b) is an amplitude normalized ideal 2nd derivative waveform $w(t)$ representing a single received chip.

data bit $[3,4]$. The duration of each monocycle pulse is typically on the order of one nanosecond (ns) and is transmitted directly without a sinusoidal carrier signal [11]. Impulse radio (IR) systems divide time into frames for each user much like conventional narrow band TDMA systems with a frame width $T_{f}$ of typically 100 to 1000 times the width of the transmitted monocycle pulse $p(t)[3,4]$. Each frame is then broken into smaller slots of $T_{c}$ seconds in which a user may transmit only one chip in his assigned time slot per frame. Not all slots in a given frame may be available for user transmission as control signals must also occupy a certain number of slots per frame.

One approach for a multiple access impulse radio system would be to allow each user to transmit his chip in a slot agreed upon by the transmitter and receiver, and to continue transmitting in the same slot for every frame until all data $\{d\}$ is transmitted. One problem with such an approach is that the added periodicity of a uniformly spaced pulse train creates spectral lines in the signal PSD [3,4]. Such spectral lines undermine the objective of a noise-like white PSD because they denote power concentrated every $\frac{1}{T_{f}}$ seconds $[3,4]$. The spectral lines allow information to be more easily intercepted and can cause interference to existing spectral occupants. A potentially more detrimental problem with periodic slotted transmission of chips is the case when two or more transmitter and receiver pairs choose to transmit in the same slot. In this case, catastrophic collisions occur for the entire 
bit transmission. To mitigate the aforementioned problems, a pseudorandom time shift is introduced into the transmitted pulse train $[3,4]$.

A pseudorandom time-hopping (TH) code $\left\{c^{k}\right\}$ represents random integer shifts in a frame according to the number of data slots $T_{c}$ available for the $k^{\text {th }}$ user's transmission. A transmitter and receiver pair must agree upon a pseudorandom $\mathrm{TH}$ code that designates the slot in which a user transmits his chip in each new frame. The introduced TH code smoothes the PSD by reducing periodicity and adds an element of privacy and security by reducing the likelihood of periodic signal interception or jamming. Of course collisions will occur in a system using a pseudorandom $\mathrm{TH}$ code if two users choose to transmit in the same slot for one frame; however, the probability of chip collisions is greatly reduced by the TH code and depending on the spreading sequence $N_{s}$, the received bit may still be correctly detected.

\subsection{Binary Pulse Position Modulation}

The modulation scheme of an impulse radio system impacts the performance in terms of Bit Error Rate (BER), spectral shaping, data rate, and hardware complexity [12]. We choose to examine Binary Pulse Position Modulation (BPPM) both theoretically and through simulation as this is the most common modulation method encountered in IR literature. Each BPPM pulse is of equal amplitude; the value of a data bit determines the position of a user's pulse inside of each transmission slot [13]. We arbitrarily define a '0' bit to modulate a pulse with no shift inside of its transmission slot and a ' 1 ' data bit to modulate a pulse shifted $\delta$ ns inside of its transmission slot.

The BPPM system is time-orthogonal if the value of $\delta$ is large enough that two nonoverlapping pulses may fit in a single transmission slot. In this case, a pulse in the first half of the slot represents a ' 0 ' bit and a pulse in the second half represents a ' 1 ' bit. The timeorthogonal system received waveforms can be demodulated with an ideal matched filter as 
all of the transmitted pulse energy can be recovered due to the time separation of the two pulse waveforms. A template waveform given by

$$
v(t)=w(t)-w(t-\delta)
$$

reduces the reference signal set and the number of threshold comparisons at the detector [3]. The orthogonal BPPM system requires a transmission slot width $T_{c}$ of approximately twice the size of the transmitted pulse waveform, which increases the transmitted frame width $T_{f}$ and decreases the system throughput given by [4, 3].

$$
R_{s}=\frac{1}{T_{s}}=\frac{1}{N_{s} T_{f}}
$$

The BPPM system throughput can be increased by decreasing the width of each transmission slot $T_{c}$ and overlapping the transmitted binary pulse waveforms in time. According to $[3,5]$, the optimal overlapped BPPM system offsets the time-shifted waveform by $\delta_{\text {opt }} n s$, the time-shift $\delta$ that minimizes the autocorrelation $R(\delta)$ given by

$$
R(\boldsymbol{\delta})=\int_{-\infty}^{\infty} w(t) w(t-\delta) d t
$$

We illustrate the ideal optimal overlapped BPPM binary received waveforms in Fig. 2.2, where $w(t)$ represents a ' 0 ' bit (top) and $w(t-\delta)$ represents a ' 1 ' bit (bottom).

The increased throughput performance of the optimal overlapped BPPM system comes at the cost of exchanging the ideal matched filter template in the case of orthogonal BPPM waveforms, to a suboptimal matched filter structure. The matched filter receiver for the optimal overlapped BPPM waveforms uses the template waveform $v(t)$ from Equation 2.2 and illustrated in Fig. 2.3, where in this case $\delta$ becomes $\delta_{\text {opt }}$. The receiver output is no longer optimal in that the total transmitted energy cannot be received in the noiseless ideal case. 


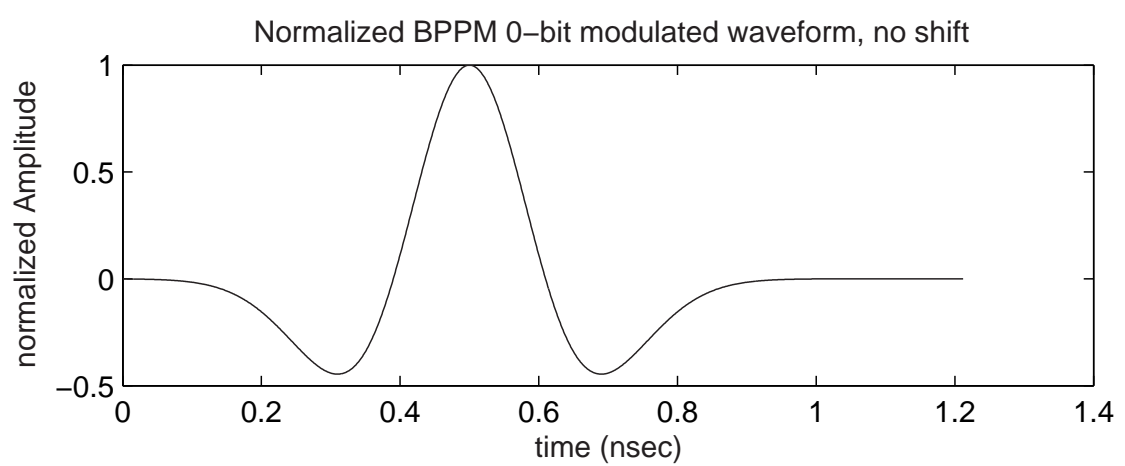

Normalized BPPM 1-bit modulated waveform, time shift by $\delta_{\text {opt }}$

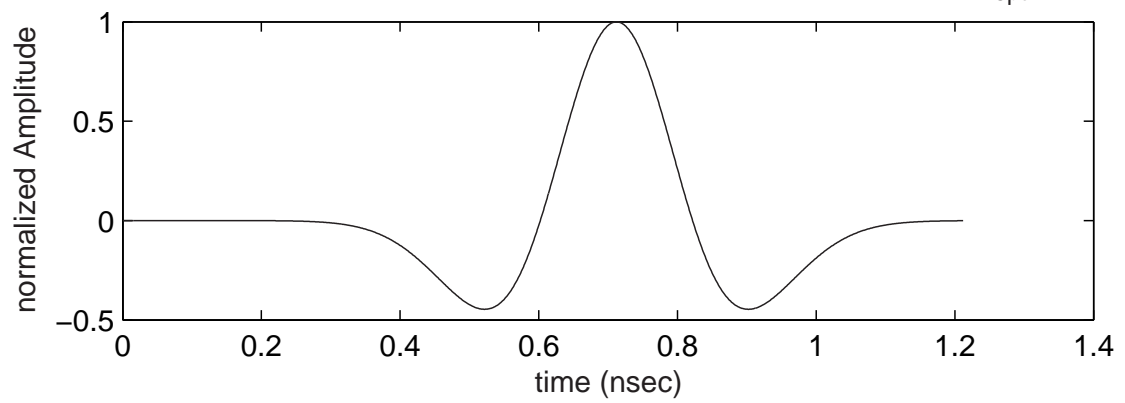

Figure 2.2: Ideal received BPPM chip waveforms representing a ' 0 ' bit (top) and ' 1 ' bit (bottom). We use optimally overlapped BPPM to offset the pulses by $\delta_{\mathrm{opt}}=0.212 \mathrm{~ns}$ as this obtains the minimum autocorrelation in Equation 2.4 [3].

The general equation for the $k^{\text {th }}$ transmitting user in a TH-BPPM impulse radio system is given by

$$
s^{(k)}(t)=\sqrt{E_{t x}^{(k)}} \sum_{j=0}^{N_{s}-1} p\left(t-j T_{f}-c_{j}^{(k)} T_{c}-\delta d_{\left[j / N_{s}\right]}^{(k)}\right)
$$

where $p(t)$ is the unit-energy transmitted waveform, $E_{t x}$ is the transmitter energy, $N_{s}$ is the number of chips per bit, $T_{f}$ is the frame length, $T_{c}$ is the slot width, and $d$ is the data bit value $[3,4]$. We illustrate the effect of each parameter from Equation 2.5 on the frame location of the transmitted pulse in Fig. 2.4. Hereafter BPPM refers to the optimally overlapped variety as increased throughput is a main objective of the networks of interest. 


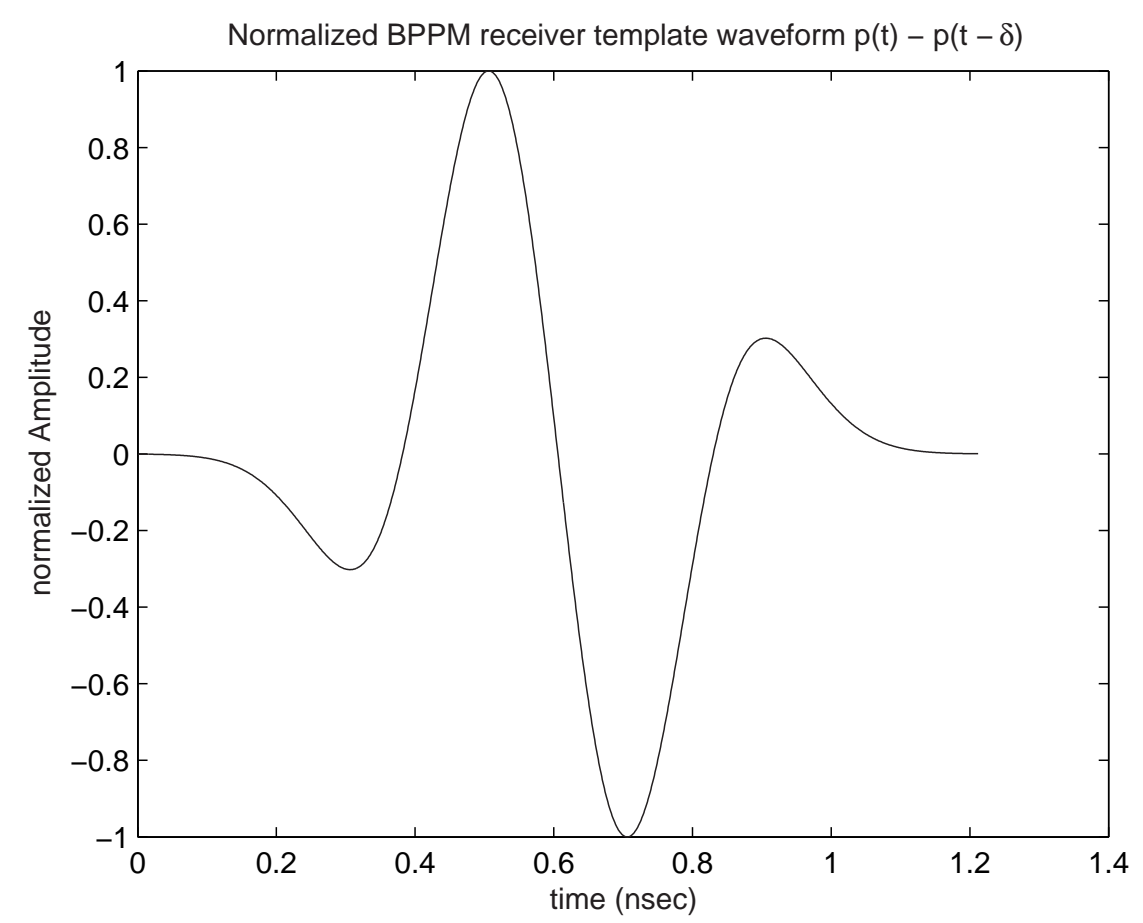

Figure 2.3: BPPM template used at the correlator receiver front-end demodulator given in Equation 2.2. The received pulse waveforms are approximately $1 \mathrm{~ns}$ in duration allowing an optimal PPM shift of $\delta_{\mathrm{opt}}=$ $0.212 \mathrm{~ns}[3,4]$.

\subsection{Autonomous Chip Discrimination Method}

Precise power control can limit large near-far ratios to improve system performance even in the presence of many co-located transceivers. Commercial cellular architectures provide power control algorithms at a base station that regulates the transmit power of all connected nodes to minimize multi-user interference [14]. The luxury of centralized power control algorithms does not apply to the ad hoc military networks of interest where survivability and vulnerability in hostile environments are of key importance [11]. Ideally, each node should therefore locally determine all transmit power adjustments without base station assistance. A coordinated power control algorithm for peer-to-peer networks was proposed in [11], but requires cooperation between nodes to continuously update one another with feedback information concerning current transmit power levels and link distances between nodes. Synchronized blanking as presented in [15] is also a possibility to limit system performance degradation due to co-located powerful users; however, the timing acquisition 


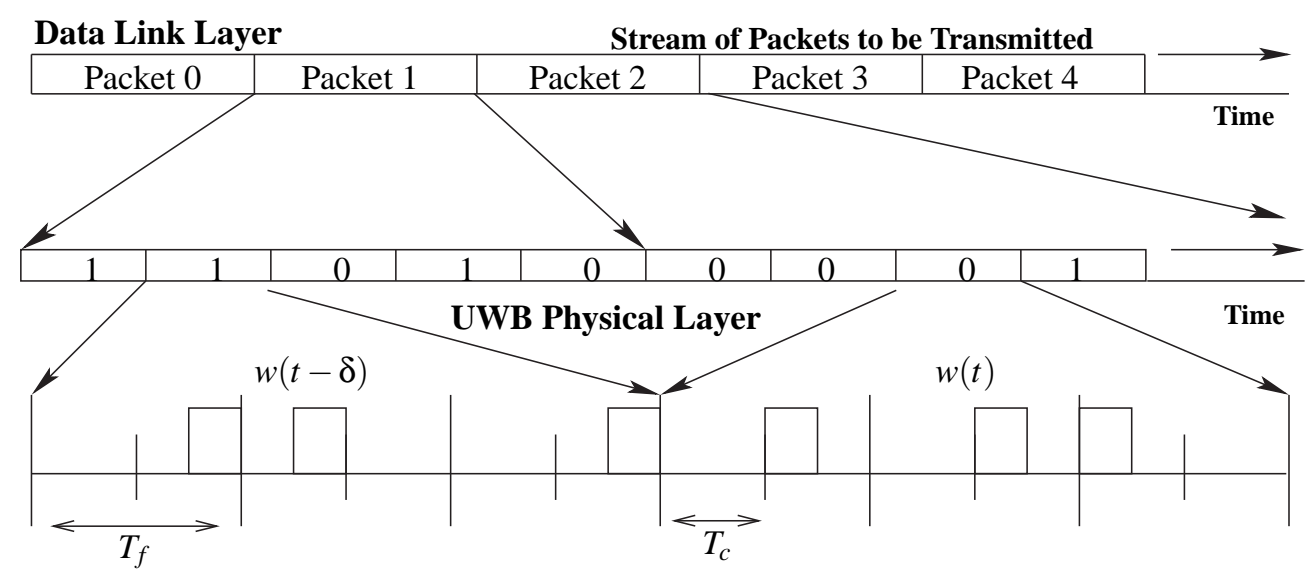

Figure 2.4: A hierarchical view of a single user's transmission at the data link and physical layers in a time-orthogonal BPPM, time-hopping impulse radio network. The physical layer transmission follows Equation 2.5 with the following parameters: $N_{s}=3$ chips per bit, $N_{s l}=2$ slots per frame, and the binary pseudorandom TH codes $c_{j}$ for the indicated 0 and 1 data bits are: $c_{b i t 1}=[1,0,1]$ and $c_{b i t}=[1,1,0]$ respectively.

required for a large number of co-located interferers quickly increases system latency to an unmanageable level. The implementation of either the coordinated power control algorithm or synchronized blanking requires a significant increase in system complexity and implicitly assumes that the interferer sources are willing to cooperate and not hostile systems [1].

An autonomous technique to mitigate the performance loss of a multi-user impulse radio ad hoc network with large near-far power ratios was proposed in [1]. The technique exploits the IR spreading sequence $N_{s}$ and applies an acceptance level to each of the $N_{s}$ matched filter output voltage samples hereafter referred to as demodulation metrics. Each individual demodulated chip metric $m$ passes through a chip discriminator which produces an output metric $m_{\text {out }}$ based the condition

$$
m_{\text {out }}= \begin{cases}0 & \text { for }|m|>|X| \\ m_{\text {in }} & \text { for }|m| \leq|X|\end{cases}
$$

where $X$ is a specified constant threshold to which all demodulated metrics are compared. In the IR system described in $[3,4]$, each of the $N_{s}$ demodulated chip metrics $m_{\text {out }}$ are 
combined to create a composite bit metric $m_{b}$ expressed mathematically as

$$
m_{b} \triangleq \sum_{n=0}^{N_{s}-1} m_{\text {out }}^{(n)}
$$

A bit estimate is calculated at the detector by comparing $m_{b}$ to thresholds defined by the chosen system modulation scheme [3]. When the system employs optimal BPPM as discussed in Section 2.1, the composite bit metric $m_{b}$ can be compared to a zero-voltage threshold at the detector with a bit estimate $\hat{b}$ based on the condition

$$
\hat{b}=\left\{\begin{array}{l}
0 \text { for } m_{b} \geq 0 \\
1 \text { for } m_{b}<0
\end{array} .\right.
$$

If chip discrimination is applied to the system as shown in Fig. 2.5, a chip metric that falls within the predefined threshold range is passed to the summer unaltered. If the chip metric exceeds the threshold, it is discarded and not contributed to the composite bit sum. The threshold is chosen such that exceptionally large chip metrics, which can only occur due to collision with a strong co-located interferer pulse, are reliably discarded. Bit errors can still occur from small overlaps in local interferer and desired pulses as well as from thermal noise when a chip metric magnitude is too small to be removed from the composite metric; however, these sources of error are not the primary concern in a network with large near-far power ratio and can furthermore be mitigated by increasing the signal to interferer ratio at the matched filter output given by

$$
S I R=\frac{N_{s} T_{f}(1-R(\delta))^{2}}{\sigma_{M}^{2} \sum_{n=1}^{N u-1}\left(E_{R X}^{(n)} / E_{R X}^{(0)}\right)}
$$

where

$$
\sigma_{M}^{2}=\int_{-\infty}^{\infty}\left[\int_{-\infty}^{\infty} w(t+\psi) v(t) d t\right]^{2} d \psi
$$

for the Gaussian channel [16, 3]. In general BER performance increases with increased 
spreading sequence $N_{S}$ for a channel with equal powered users and thermal noise, but at the cost of decreasing the system throughput according to Equation 2.3.

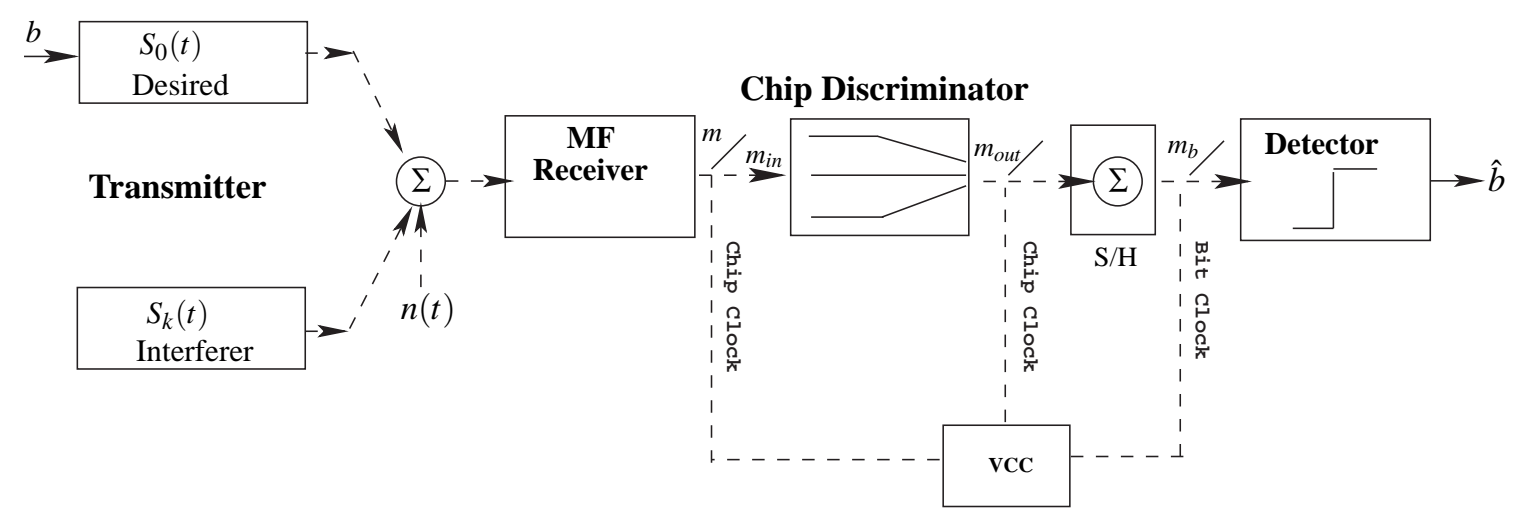

Figure 2.5: Block diagram of a basic multi-user IR system in the presence of AWGN $n(t)$ as described in [3]. A chip discriminator device is added at the matched filter output to remove samples from the bit sum that exceed a predefined threshold [1]. $N_{s}$ received chip metrics are added together before the result is sampled and sent to the detector for a bit estimation.

In an IR system without chip discrimination, increasing $N_{s}$ decreases the BER floor caused by distant equal-power users and thermal noise degradation; it does not alleviate performance loss due to powerful near interferer pulse collisions. When a powerful interferer arrives inside of a chip's correlation window, the chip metric magnitude $|m|$ grows according to the demodulated interferer energy. A corrupted chip metric $m$ continues to corrupt the bit metric $m_{b}$ according to Equation 2.7 since the large interferer contribution usually overwhelms the entire sum of uncorrupted $m$. In the optimal BPPM system, once the sign of the composite metric $m_{b}$ becomes corrupted by a large interferer energy contribution, increasing its magnitude is still erroneous. The chip discrimination method requires at least $N_{s}=2$, and in general a $N_{s}$ value between 10 and 100 is chosen that balances BER performance and system throughput. Local interference is the primary source of performance degradation in the large near-far power ratio environments where chip discrimination is necessary. When the transmitted data bit is spread over a large number of chips, not only can the corrupted chips be more reliably discarded at the chip discriminator, but the BER floor is reduced such that errors due to other noise sources are minimized [1]. 
The chip-discrimination technique presented in [1] applies to the theoretical Gaussian channel where the desired and multi-user signals randomly arrive at the receiver via a single path corrupted only by multi-user interference and thermal noise. The remaining chapters of this thesis shall extend the chip-discrimination technique presented in [1] as a solution to mitigate the system performance loss due to large near-far power ratios in an indoor multipath environment. 


\section{Chapter 3}

\section{The Multipath Indoor UWB Channel}

The focus of channel model research as applied to UWB communications is to develop and apply a statistical model to a system simulation that captures time-varying distortions such as attenuation, phase jitter, and intersymbol interference (ISI). Performance results for multi-user, BPPM impulse radio systems that approximate multi-user interference as a Gaussian random process are well known and widely available in literature including $[3,4,17]$; results are not so easily found reporting multi-user impulse radio (IR) system performance through multipath channels. Even in dense multi-user IR networks where the Gaussian approximation can be quite accurate, system performance in the presence of a channel model can reveal parameter sensitivities not demonstrated by the Gaussian channel.

The lack of UWB channel models in literature, especially outdoor models, can be attributed to the extensive collections of data that must be measured to accurately quantify the small-scale fading experienced in a particular environment. After the data has been collected, equally difficult is the process of selecting a statistical distribution that consistently adheres to the basic channel properties exhibited by the measured data while remaining tractable and efficient in terms of simulation time. Finally the data measurements are specific to the measured environment and inherently depend on the underlying measurement 
equipment.

Once researchers find a statistical distribution that closely matches the measured data, the model is applicable only to similar environments. The UWB channel measurement campaigns to date have examined the indoor office building environment as they have been motivated by the IEEE 802.15.3a subgroup's efforts to adopt a standard indoor UWB channel model [9]. Results published by the IEEE 802.15.3a subgroup demonstrated that at small transmitter and receiver separation distances, approximately 0 - 4 meters, channel effects can be modeled accurately by altering the parameters of the existing wide band Saleh-Valenzuela (S-V) channel model [7, 8, 9].

\subsection{Saleh-Valenzuela Channel Model}

The Saleh-Valenzuela (S-V) Model is a statistical, complex lowpass tapped delay line channel model that closely fits NLOS channel measurements obtained using $1.5 \mathrm{GHz}$ radarlike pulses of $10 \mathrm{~ns}$ duration in an office-building environment [6]. In general the indoor NLOS measurements demonstrated that multipath components (MPCs) arrived in groups called clusters created by large objects such as walls or furniture that produce attenuated replicas of the original signal. Each cluster then contained many more severely attenuated signal replicas called rays produced by smaller objects in the environment [6].

We illustrate the idea of clusters and rays with a typical power delay profile (PDP) example produced by an impulse response realization magnitude squared $|h(t)|^{2}$ in Fig. 3.1. The multipath spread of the channel $T_{m}$ denotes the range of multipath delay values $\tau$ over which the PDP is nonzero [18]. To model the observed clustering effect, the S-V model uses a Poisson process with a rate $\Lambda$ to choose the cluster arrival times and a second Poisson process with a rate $\lambda$ to choose the arrival times of the attenuated rays occurring inside of a cluster [6]. By definition of the Poisson process, the clusters and rays both have exponentially distributed interarrival times with rates $\Lambda$ and $\lambda$ respectively, where the first ray of the first cluster always arrives at time $t=0 \mathrm{~ns}$. The independent exponential interarrival prob- 


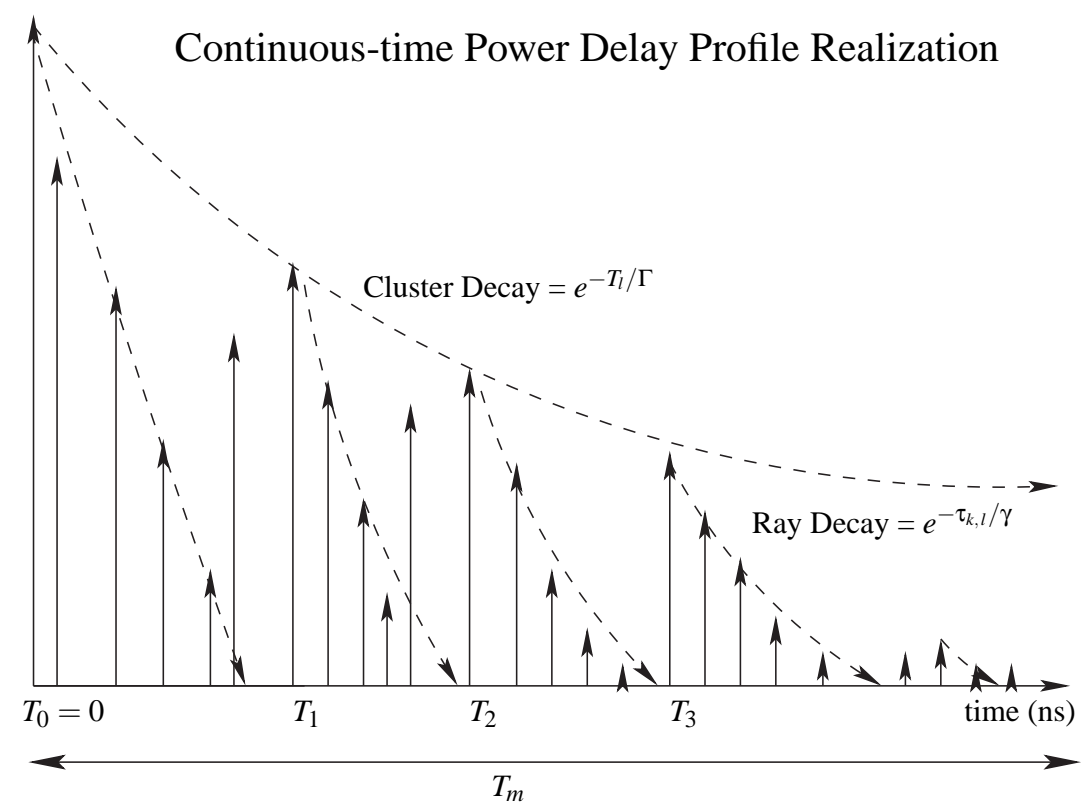

Figure 3.1: A typical Power Delay Profile (PDP) demonstrates the clusters and rays created by multipath reflections and refractions from large and small objects in an indoor environment $[6,5,19]$. The cluster arrival times are denoted $T_{l}$ and the arrival time of the $k^{t h}$ ray within the $l^{t h}$ cluster is denoted $\tau_{k, l}$.

ability density functions (PDFs) for the cluster and ray distributions are given respectively by

$$
\begin{gathered}
p\left(T_{l} \mid T_{l-1}\right)=\Lambda e^{-\Lambda\left(T_{l}-T_{l-1}\right)}, \quad l>0 \\
p\left(\tau_{k, l} \mid \tau_{(k-1), l}\right)=\lambda e^{-\lambda\left(\tau_{k, l}-\tau_{(k-1), l}\right)}, \quad k>0
\end{gathered}
$$

where $T_{l}$ represents the arrival time of the $l^{\text {th }}$ cluster and $\tau_{k, l}$ is arrival time of the $k^{\text {th }}$ ray in the $l^{t h}$ cluster. The ray time delays $\tau_{k, l}$ are with respect to the cluster in which they are contained such that the first ray of any cluster begins at time $\tau_{k, l}=0 \mathrm{~ns}$ with respect to the cluster time $T_{l}[6]$. We assume that each cluster consists of many rays such that in general $\lambda \gg \Lambda$.

Equipment limitations in the original measurement campaign prevented many weak rays from being resolved within the equipment time resolution [6]. This fact is exploited by assuming that the resolved ray is actually the sum of many independent rays arriving within the system time resolution. Under this assumption, the multipath coefficients $\beta_{k, l}$ 
can be modeled as Rayleigh distributed with PDF expressed as

$$
p\left(\beta_{k, l}\right)=\left(2 \beta_{k, l} / \Omega_{k, l}\right) e^{\left(-\beta_{k, l}^{2} / \Omega_{k, l}\right)}
$$

where

$$
\Omega_{k, l}=E\left[\beta_{k, l}^{2}\right]=\Omega_{00} e^{\left(-T_{l} / \Gamma\right)} e^{\left(-\tau_{k, l} / \gamma\right)}
$$

The measured receiver employed a square-law envelope detection process which does not preserve the resolved signal's phase information [14]; the phases are therefore modeled as statistically independent, uniformly distributed random variables $\theta_{k, l}$ between 0 and $2 \pi$. An exponential power decay of both rays and clusters models the large dynamic range of attenuation experienced in measurements and is controlled by the exponentially decreasing average power gain $\Omega_{k, l}$ given by Equation 3.4. The power-delay time constants $\Gamma$ and $\gamma$ determine the intensity of decay for each cluster and ray respectively according to a ray's relative arrival time [6].

The $i^{t h}$ realization of the complex lowpass impulse response $h_{S V}^{i}(t)$ can be expressed as

$$
h_{S V}^{i}(t)=\sum_{l=0}^{\infty} \sum_{k=0}^{\infty} \beta_{k, l} e^{j \theta_{k, l}} \delta\left(t-T_{l}-\tau_{k, l}\right) .
$$

We can consider $h_{S V}^{i}(t)$ as a continuous-time impulse response as there is no quantization implied in this equation; a given ray can arrive at any time within the observation time of the channel [6]. The continuous-time impulse response $h_{S V}^{i}(t)$ must be converted to a discrete-time impulse response using the methods described in Section 3.2.2 to limit the system resolution and create a useful simulation model.

\subsection{IEEE 802.15.3a Modified S-V Channel Model}

The IEEE 802.15.3a subgroup recently adopted a UWB short-range indoor channel model created by modifying the wide band S-V model in Section 3.1 to match measured 
data from office buildings similar to those measured in [6]. The IEEE 802.15.3a channel model, hereafter referred to as "the channel model," stochastically creates LOS and NLOS impulse responses analogous to those typically found in an indoor home or office environment with a transmitter and receiver separation of 0-4 meters $[7,8,9]$.

The authors modified the original S-V model by comparing measured UWB data and the S-V Model using a variety of different multipath characterization parameters including: mean excess delay, RMS delay spread, PDP, and number of multipath components within $10 \mathrm{~dB}$ of the peak multipath arrival $[7,8,9]$. The model parameters were then calibrated until the channel model parameters sufficiently matched the measured data parameters. Here we describe the basic modifications made to the original S-V model from Section 3.1 to arrive at the UWB IEEE 802.15.3a model. The remaining sections include: an equivalent discrete-time channel model, the limitations of the channel model, the specific system channel model modifications, and the channel multipath characterization results.

\subsubsection{Modifications to the S-V Model}

The "clustering" effect discussed in Section 3.1 was observed in the UWB indoor environments measured according to $[7,8,9]$. The dual Poison processes that model the cluster and ray arrival times are used unaltered from the original S-V model with the cluster and ray arrival time PDFs given in Equations 3.1 and 3.2 respectively [7, 8, 9]. The precision of the UWB measuring equipment as well as the measuring method is different in this case. The results are based on a frequency sweep of 2-6 GHz which yields a minimum path resolution of $0.167 \mathrm{~ns}$ [9]. In many cases the received signal was sampled directly without loss of information as in the original S-V model experiment where the phase information was lost and necessarily modeled as a uniformly distributed random variable $\theta_{k, l}$. The IEEE 802.15.3a channel model replaces the phase $\theta_{k, l}$ distributed between 0 and $2 \pi$ with a random phase $p_{k, l}$ that takes the equiprobable values \pm 1 . This allows the channel model $h(t)$ to be real-valued while capturing the effects of the $\pi$ radian phase change that occurs 
when the multipath components (MPCs) are reflected $[8,9]$.

It is well known that in narrow band systems, a large number of multipath components can be modeled as Rayleigh distributed using the central limit theorem because the multipath reflections are not resolvable when the system bandwidth is less than the channel coherence bandwidth [20]. The result is a large number of MPCs that arrive at the receiver within its resolution time such that the envelope of the signal can be modeled as a Rayleigh random variable. This is usually not the case in an impulse radio system where the system bandwidth exceeds the channel coherence bandwidth to increase the MPC resolution. Hence less reflections arrive at the receiver during a single resolution time [20]. The UWB indoor measurement campaign results presented in $[8,9]$ determined that the Rayleigh distribution did not accurately model the channel tap coefficients for a UWB system; however, the lognormal distribution with PDF given by

$$
f_{X}(x)= \begin{cases}\frac{1}{\sqrt{2 \pi} \sigma x} e^{-\frac{\left(10 \log _{10}(x)-\mu\right)^{2}}{2 \sigma^{2}}} & \text { for }(x \geq 0) \\ 0 & \text { otherwise }\end{cases}
$$

sufficiently models the channel tap coefficient amplitude fluctuations as previously recommended as an alternate solution to the generic S-V channel model in [6]. In Equation 3.6, a Gaussian distributed random variable $Y$ exists with mean $\mu$ and variance $\sigma^{2}$ as defined by the notation $\mathcal{N}\left(\mu, \sigma^{2}\right)$ with $\mathrm{dB}$ units. The lognormal random variable $X$ is then related to $Y$ through the transformation $Y=10 \log _{10}(X)$, assuming $Y$ represents power [18].

We model the channel tap coefficients $\alpha_{k, l}$ as the product of three individual and independent components expressed as

$$
\alpha_{k, l}=p_{k, l} \xi_{l} \beta_{k, l}
$$

where $\xi_{l}$ is a large-scale fading coefficient, $\beta_{k, l}$ is a small-scale fading coefficient, and $p_{k, l}$ represents the equiprobable phase term $[7,8,9]$. The large-scale fading term $\xi_{l}$ is lognormally distributed with Gaussian standard deviation $\sigma_{1}$. This imposes a constant 
attenuation on all arriving rays or paths arriving inside of each cluster. The small-scale fading term $\beta_{k, l}$ is also lognormally distributed with Gaussian standard deviation $\sigma_{2}$, but is independently chosen for each ray to provide individual amplitude fluctuations. Because the large-scale and small-scale fading random variables have the same distributions, we can group the terms together as

$$
\left|\xi_{l} \beta_{k, l}\right|=10^{\left(\mu_{k, l}+n_{1}+n_{2}\right) / 20}
$$

where $n_{s} \sim \mathcal{N}\left(0, \sigma_{s}^{2}\right)$ and $\mu_{k, l}=\frac{10 \ln \left(\Omega_{0,0}\right)-10 T_{l} / \Gamma-10 \tau_{k, l} / \gamma}{\ln (10)}-\frac{\left(\sigma_{1}^{2}+\sigma_{2}^{2}\right) \ln (10)}{20}$.

The resulting distribution for $\left|\xi_{l} \beta_{k, l}\right|$ is lognormal with Gaussian mean $\mu_{k, l}$ and Gaussian variance $\sigma_{1}^{2}+\sigma_{2}^{2}[7,8,9]$.

The channel coefficient Gaussian mean $\mu_{k, l}$ depends on the average power gain $\Omega_{0,0}$ of the first ray arrival of the first cluster exactly as found in the S-V model to evaluate $\Omega_{k, l}$ in Equation $3.4[7,8,9]$. In general we assume the term $\Omega_{0,0}$ is unity as each impulse response realization is normalized to have unit energy such that no artificial gain is introduced into channel experiments. The average power gain of each MPC is determined exactly as in Equation 3.4 where the S-V MPC $\beta_{k, l}=\left|\xi_{l} \beta_{k, l}\right|$.

The effects of large-scale attenuation that occurs over many different measurement locations with the same transmitter-receiver (Tx-Rx) separation is modeled as a lognormally distributed random variable $X_{i}$ expressed as

$$
10 \log _{10}\left(X_{i}\right) \sim \mathcal{N}\left(\mu_{x}, \sigma_{x}^{2}\right)
$$

where

$$
\mu_{x}=\frac{10 \ln (G)}{\ln (10)}-\frac{\sigma_{x}^{2} \ln (10)}{20} .
$$

The unit energy impulse response is scaled by $X_{i}$, which remains constant over each channel realization $[7,8,9] . X_{i}$ has Gaussian mean $\mu_{x}$ which is determined similarly to the Gaussian 
mean $\mu_{k, l}$ of the channel tap coefficients in Equation 3.9. The lognormal mean of $X_{i}$ is equal to the average channel energy gain $G$ and the Gaussian mean $\mu_{x}$ can be found using Equation 3.10 in terms of $G$ and $\sigma_{x}^{2}[21,5]$. We can determine the average channel energy gain $G$ through simulation by averaging the energy contained in all $L$ resolvable ray paths expressed mathematically as

$$
G=E\left[G^{i}\right]
$$

where

$$
G^{i} \triangleq \sum_{n=1}^{L}\left|a_{n}^{i}\right|^{2}
$$

$G$ can also be determined analytically using the large-scale fading parameters in Table 3.1 given by

$$
G=G_{0} / D^{\alpha_{p}}
$$

where

$$
G_{0}=10^{-A_{0} / 10}
$$

To analytically calculate the average channel energy $G$, the Tx-Rx distance (D) must be known and the parameters of Table 3.1 which quantify the large-scale path loss must be matched to the modeled environment $[21,5] . D_{0}$ is the reference Tx-Rx separation of 1 meter and $A_{0}$ is the reference path loss that occurs with Tx-Rx separation of $D_{0}$ meters. The path-loss exponent $\alpha_{p}$ characterizes the severity of path loss attenuation depending on the particular environment obstructions; the free space path loss exponent is two and increases according to the number of large signal obstructions. Table 3.1 lists the common indoor values for parameters $A_{0}$ and $\alpha_{p}$ [5].

The $i^{\text {th }}$ generated impulse response realization can be represented as

$$
h_{i}(t)=X_{i} \sum_{l=0}^{N_{c l s}-1} \sum_{k=0}^{N_{\text {rays }}-1} \alpha_{k, l}^{i} \delta\left(t-T_{l}^{i}-\tau_{k, l}^{i}\right)
$$

where the total number of clusters and rays present in the $i^{t h}$ realization are given by $N_{c l s}$ 
and $N_{\text {rays }}$ respectively $[7,8,9]$.

Each of the parameters that compose the IEEE 802.15.3a channel model are described in Table 3.2 along with the suggested necessary parameter values listed in Table 3.3 taken directly from [8]. The four channel models defined in Table 3.3 are not arbitrary and can in fact be found throughout literature referenced using simply CM1, CM2, CM3, or CM4. We also adopt this notation and from this point we reference each channel model according to its standard label in Table 3.3.

Table 3.1: Common indoor parameters for large-scale path loss attenuation factor $A_{0}$ and path loss exponent $\alpha_{p}[5,21]$.

\begin{tabular}{||l|c|c||}
\hline \hline Channel Model Parameters & LOS & NLOS \\
\hline$A_{0}[\mathrm{~dB}]$ (Path loss at reference distance $\left.D_{0}=1 \mathrm{~m}\right)$ & 47 & 51 \\
\hline$\alpha_{p}$ (Path loss exponent) & 1.7 & 3.5 \\
\hline
\end{tabular}

\subsubsection{Discrete-Time Impulse Response}

The IEEE 802.15.3a channel model presented in Section 3.2 can be considered a continuous-time channel model; The clusters and rays have statistically independent exponential interarrival times given by Equations 3.1 and 3.2, where the arrival time is as precise as the simulation tool from which it is chosen. In practice this is not the case since multipath components cannot be resolved with infinite resolution; the received multipath components that arrive adjacently within a delay less than the received pulse width $T_{p}$ cannot be individually resolved. To limit the system resolution according to the received pulse width $T_{p}$, we convert the continuous-time impulse response $h_{i}(t)$ from Equation 3.13 to an equivalent discrete-time impulse response.

We divide the time axis into bins with the bin width defined as the resolution of the channel or the largest time interval over which the receiver is not capable of distinguishing separate paths [5]. The bin width is chosen to be $T_{p}$ which models the fine resolution of multipath components by restricting the fading of interfering reflections [7]. We quantize the continuous-time impulse response components to a particular bin, where multiple 
Table 3.2: IEEE 802.15.3a UWB channel model parameters given with mathematical symbol and functional description $[7,8,9,5]$.

\begin{tabular}{|c|c|}
\hline Parameters & " Description \\
\hline$\Lambda[1 / \mathrm{nsec}]$ & $\begin{array}{l}\text { Cluster arrival rate or inverse mean of the exponentially distributed } \\
\text { cluster interarrival times. }\end{array}$ \\
\hline$\lambda[1 / \mathrm{nsec}]$ & $\begin{array}{l}\text { Ray arrival rate or inverse mean of the exponentially distributed ray } \\
\text { interarrival times. }\end{array}$ \\
\hline$T_{l}^{i}[\mathrm{nsec}]$ & Arrival time of the $l^{\text {th }}$ cluster of the $i^{\text {th }}$ impulse response realization. \\
\hline$\tau_{k, l}^{i}$ & $\begin{array}{l}\text { Arrival time of the } k^{\text {th }} \text { ray relative to the } l^{\text {th }} \text { cluster in the } i^{\text {th }} \text { impulse } \\
\text { response realization. }\end{array}$ \\
\hline $\bar{\Gamma}[\mathrm{nsec}]$ & Exponential decay factor imposed on a given cluster. \\
\hline$\gamma[\mathrm{nsec}]$ & $\begin{array}{l}\text { Exponential decay factor imposed on each ray inside of a given } \\
\text { cluster. }\end{array}$ \\
\hline$\alpha_{k, l}^{i}$ & $\begin{array}{l}\text { Multipath gain coefficient or lognormally distributed amplitude term } \\
\text { for the } k^{\text {th }} \text { ray inside the } l^{\text {th }} \text { cluster of the } i^{\text {th }} \text { generated impulse } \\
\text { response realization. }\end{array}$ \\
\hline$\beta_{k, l}^{i}$ & $\begin{array}{l}\text { Lognormally distributed small-scale fading coefficient of the } k^{\text {th }} \text { ray } \\
\text { inside the } l^{\text {th }} \text { cluster of the } i^{\text {th }} \text { impulse response realization. }\end{array}$ \\
\hline$\xi_{l}^{i}$ & $\begin{array}{l}\text { Lognormally distributed large-scale fading coefficient of the } l^{\text {th }} \text { cluster } \\
\text { of the } i^{t h} \text { impulse response realization. }\end{array}$ \\
\hline$p_{k, l}$ & $\begin{array}{l}\text { Equiprobable oscillating } \pm 1 \text { sign term to account for multipath } \\
\text { reflections that result in a phase change on } \pi \text {. }\end{array}$ \\
\hline$\Omega_{0,0}^{i}$ & $\begin{array}{l}\text { Average power gain of the first ray arriving in the first cluster of the } \\
i^{t h} \text { impulse response realization. In general if we normalize the energy } \\
\text { of the } i^{t h} \text { impulse response to unity, we can neglect the term } \\
\Omega_{0,0}^{i}=E\left[\left(\alpha_{0,0}^{i}\right)^{2}\right] \text {. }\end{array}$ \\
\hline$\sigma_{1}[\mathrm{~dB}]$ & $\begin{array}{l}\text { Gaussian Standard Deviation of lognormally distributed cluster fading } \\
\text { term. }\end{array}$ \\
\hline$\sigma_{2}[\mathrm{~dB}]$ & $\begin{array}{l}\text { Gaussian Standard Deviation of lognormally distributed ray fading } \\
\text { term. }\end{array}$ \\
\hline$X_{i}[\mathrm{~dB}]$ & $\begin{array}{l}\text { A constant Lognormal shadowing term over the entire } i^{\text {th }} \text { impulse } \\
\text { response realization. }\end{array}$ \\
\hline$\sigma_{x}[\mathrm{~dB}]$ & $\begin{array}{l}\text { Gaussian Standard Deviation of lognormal shadowing term over an } \\
\text { entire impulse response realization. }\end{array}$ \\
\hline
\end{tabular}

pulses that quantize to the same bin are added together [8]. The discrete-time signal is passed through an anti-aliasing filter such that the resulting component in each bin reflects the energy of all continuous-time components arriving inside of a $T_{p}$ ns window $[8,9]$. The resulting taps of the tapped delay line channel model are spaced at approximately integer 
Table 3.3: IEEE 802.15.3a UWB channel model parameters and suggested simulation values from [8, 9].

\begin{tabular}{||l|l|l|l|l||}
\hline \hline Channel Model Parameters & CM 1 & CM 2 & CM 3 & CM 4 \\
\hline$\Lambda[1 /$ nsec] (Cluster arrival rate) & 0.0233 & 0.4 & 0.067 & 0.067 \\
\hline$\lambda[1 /$ nsec] (Ray arrival rate) & 2.5 & 0.5 & 2.1 & 2.1 \\
\hline$\Gamma[$ nsec $]$ (Cluster decay factor) & 7.1 & 5.5 & 14.00 & 24.00 \\
\hline$\gamma[$ nsec $]$ (Ray decay factor) & 4.3 & 6.7 & 7.9 & 12 \\
\hline$\sigma_{1}[\mathrm{~dB}]$ & 3.4 & 3.4 & 3.4 & 3.4 \\
\hline$\sigma_{2}[\mathrm{~dB}]$ & 3.4 & 3.4 & 3.4 & 3.4 \\
\hline$\sigma_{x}[\mathrm{~dB}]$ & 3.0 & 3.0 & 3.0 & 3.0 \\
\hline
\end{tabular}

multiples of the inverse pulse bandwidth $\frac{1}{T_{p}}$.

The generic discrete-time impulse response $h_{g}(t)$ is given by

$$
h_{g}(t)=\sum_{k=0}^{L-1} a_{k} \delta(t-k \Delta \tau)
$$

where all continuous-time impulse response components are quantized into a maximum of $L$ bins. The discrete-time impulse response $h_{g}(t)$ is composed of discrete components $a_{k}$ spaced every $k \Delta \tau$ seconds, where $k$ is a positive integer and $\Delta \tau$ is the bin duration [5]. We rewrite the continuous-time impulse response $h_{i}(t)$ from Equation 3.13 as a discrete-time impulse response expressed as

$$
h_{i}(t)=X_{i} \sum_{n=0}^{L-1} \alpha_{n}^{i} \delta\left(t-n T_{p}\right)
$$

In this case $\alpha_{n}^{i}$ is the amplitude of the discrete-time impulse response component in the $n^{\text {th }}$ bin of the $i^{\text {th }}$ channel realization. $\alpha_{n}^{i}$ represents the sum of all continuous-time components $\alpha_{k, l}$ from Equation 3.13 that quantize into the $n^{\text {th }}$ discrete-time bin [7, 8, 9]. The discrete-time impulse response $h_{i}(t)$ spaces all components as isolated arrivals every $T_{p} \mathrm{~ns}$ beginning at time $t=0 \mathrm{~ns}$. All future references to the impulse response $h(t)$ refer to Equation 3.15, which is used for subsequent channel model experiments. Table 3.4 presents a short description of the parameters that compose the discrete-time IEEE 802.15.3a channel 
model.

Table 3.4: IEEE 802.15.3a discrete-time channel model parameters given with mathematical symbol and functional description $[7,8,9,5]$.

\begin{tabular}{||l|l||}
\hline \hline Parameters & Description \\
\hline$\alpha_{n}^{i}[\mathrm{~V}]$ & $\begin{array}{l}\text { The real amplitude of the discrete-time impulse response component } \\
\text { that constitutes the sum of all continuous-time components } \alpha_{k, l} \\
\text { that quantize into the } n^{\text {th }} \text { discrete-time bin of the } i^{t h} \text { channel } \\
\text { realization. }\end{array}$ \\
\hline$\tau_{n}^{i}[\mathrm{nsec}]$ & $\begin{array}{l}\text { The arrival time of the } n^{t h} \text { discrete-time impulse response } \\
\text { component of the } i^{t h} \text { channel realization. }\end{array}$ \\
\hline
\end{tabular}

\subsubsection{IEEE 802.15.3a Channel Model Limitations}

Engineering tradeoffs always exist such that the best solution is an optimization problem to fulfill the system requirements as closely as possible within the implementation constraints. The IEEE 802.15.3a channel model is no different in that it captures the essence of an UWB multipath channel at the cost of oversimplification in certain areas. The purpose of this section is not to enumerate the flaws in the channel model, but rather to clearly state the underlying limits that influenced many of the assumptions made throughout the work presented in this thesis.

The most important limitation in modeling a UWB system with the IEEE 802.15.3a channel model comes from its lack of correlation between channel realizations. The impulse response is considered time-invariant over the transmission of a data packet with no time-variability incorporated into the model [9]. In fact, channel realizations are considered statistically independent. This may not be such a limitation in a single-user network where we can assume that the desired user's velocity is such that the channel remains approximately constant over one data packet transmission; however, in the multi-user system case that we investigate, there exists some channel correlation between the desired and interferer channels at the short Tx-Rx distances for which the channel model is valid. 
The cluster and ray arrival rates are delay-invariant and remain constant over all realizations of a given channel model regardless of the excess delay of the multipath components. This is not necessarily true as a varying excess delay in general affects the MPC arrival rates [8]. Equivalently if the interarrival time between two clusters is small, the ray arrival rate $\lambda$ should decrease as there are fewer possible propagation paths than if a large delay existed between clusters which would constitute increasing $\lambda$. However for model simplicity and computational efficiency, the cluster and ray arrival rates are not dynamic.

Although the aforementioned channel model limitations restrict its use in some detailed experiments, the model demonstrates the fundamental degradations consistent with indoor UWB measurements of a multipath environment. The limitations can in most cases be justified by a reduction in simulation complexity and increased efficiency in terms of computational time.

\subsubsection{Specific Modifications}

The discrete-time IEEE 802.15.3a channel model as presented in Section 3.2.2 can be used directly in computer simulation, but simple modifications specific to the network model presented in Chapter 4 can improve simulation performance without significant loss of accuracy. Here we briefly describe the modifications made to the simulated channel model that may impact the results presented throughout this thesis. We describe the generic methodology presented in $[8,9]$ when possible, followed by any necessary justification for modifying the generic channel model.

We use the waveform $w(t)$ illustrated in Figure 2.1(b) with pulse width $T_{p} \approx 1 \mathrm{~ns}$, such that the minimum path resolution becomes equal to $T_{p}$. Increasing the path resolution or bin width increases the probability of multiple rays quantizing to the same bin. The statistical multipath characterization parameters for the channel change accordingly as $T_{p}$ increases. Tables 3.5 and 3.6 in Section 3.2.5 list the statistical multipath parameters reported in [8, 9], but calculated from the modified discrete-time channel model described here with bin width 
$T_{p} \approx 1 \mathrm{~ns}$.

There exists no clear definition in $[8,9]$ concerning the truncation time or observation time of a given impulse response realization. In general the exponential decay of the impulse response imposed by the average power gain $\Omega_{k, l}$ implies that largest magnitude components have an arrival time close to $t=0 \mathrm{~ns}$. There exists a ray arrival time $\tau_{k, l}$ for which the corresponding ray with magnitude $\left|\alpha_{k, l}\right|$ is approximately zero. This ray arrival time $\tau_{k, l}$ can be considered the multipath spread $T_{m}$ of the channel as no more multipath components exist from the initial channel excitation [18]. Through simulation we find that no significant MPCs exist beyond $\tau_{k, l}=150 \mathrm{~ns}$, even in the case of a single cluster over the entire impulse response. We choose to truncate all impulse response realizations with cluster or ray arrival times that exceed $t=150 \mathrm{~ns}$, hereafter referred to as the observation time of the channel.

The largest magnitude MPCs are the most important in terms of increasing the signalto-noise ratio (SNR) at the receiver input. The extremely small magnitude components waste system-tracking and digital signal processing (DSP) resources as in most cases the system noise overwhelms these components such that they are received in error. Furthermore these small components are a potential source of system ISI that causes distortion in the received multipath waveforms. We therefore filter each continuous-time impulse response realization by removing any components with power less than $25 \mathrm{~dB}$ of the maximum MPC power. The filtering process saves system resources by tracking only the components that are most likely to provide increased diversity gain and improves system efficiency by removing unnecessary signal processing of very low-power components.

We conclude this section by providing an illustration of example impulse response realizations created using the techniques presented throughout this chapter. We include two continuous-time and corresponding discrete-time channel realizations using CM1 parameters in Figs. 3.2(a) and 3.2(b) and CM2 parameters in Figs. 3.3(a) and 3.3(b). The LOS impulse response with CM1 parameters has a larger number of average ray arrivals than the CM2 impulse response. This can be inferred from CM1's larger ray interarrival rate $\lambda$ in 


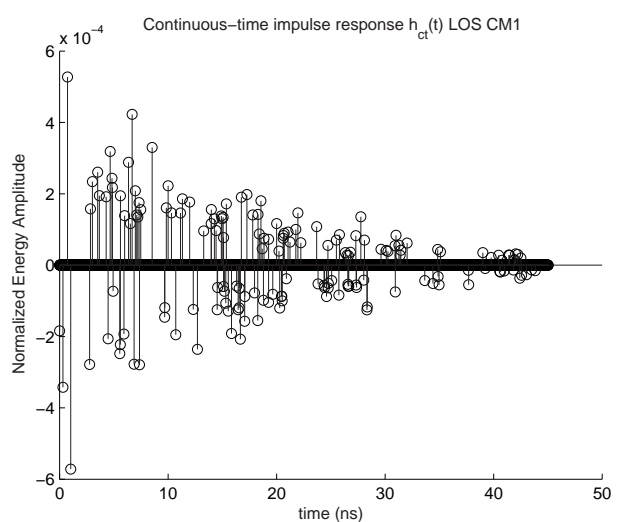

(a)

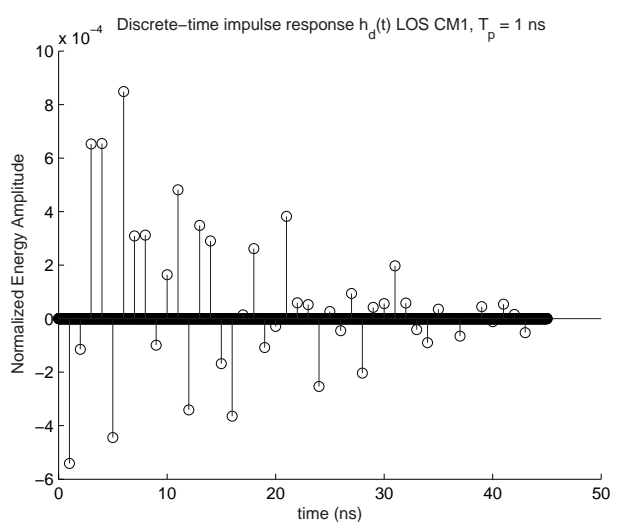

(b)

Figure 3.2: Fig. 3.2(a) illustrates a realization of the CM1 continuous-time impulse response. Fig. 3.2(b) is the equivalent discrete-time impulse response for the CM1 realization in Fig. 3.2(a) with bin width $T_{p}=1 \mathrm{~ns}$.

Table 3.3 and demonstrated in the simulated multipath channel results in Table 3.5 for the mean number of resolvable paths $\mathrm{E}[\mathrm{NP}]$. The total multipath gain $G$ differs in both realizations; as expected, the CM1 realization has larger gain than the CM2 realization because of increased large-scale attenuation in the NLOS channel.

\subsubsection{Multipath Characteristics of the IEEE 802.15.3a Channel}

A standard set of statistical multipath characterization parameters is needed to consistently quantify different multipath channels for comparison. We characterize the simulated multipath channel impulse response using the following statistical parameters: mean excess delay $\left(\overline{\tau_{i}}\right)$, rms delay spread $\left(\overline{\tau_{i}^{2}}\right)$, number of paths with energy within $10 \mathrm{~dB}$ of the strongest path $\left(\mathrm{NP}_{10 \mathrm{~dB}}\right)$, number of largest energy paths to capture 85 percent of the channel energy $\left(\mathrm{NP}_{85}\right)$, the mean channel energy $\left(E\left[\varepsilon_{\text {channel }}\right]\right)$ in $\mathrm{dB}$, and the mean number of resolvable paths $\mathrm{E}[\mathrm{NP}][14,8]$. These parameters are important because they serve as the bridge between measured data and the channel parameters given in Table 3.3. In fact researchers created the IEEE 802.15.3a channel model based on calibrating the channel model parameters until the measurement data and channel model statistical multipath parameters agreed. We describe the most common statistical multipath parameters followed by parameter evaluation for the IEEE 802.15.3a discrete-time channel model through computer simulation. 


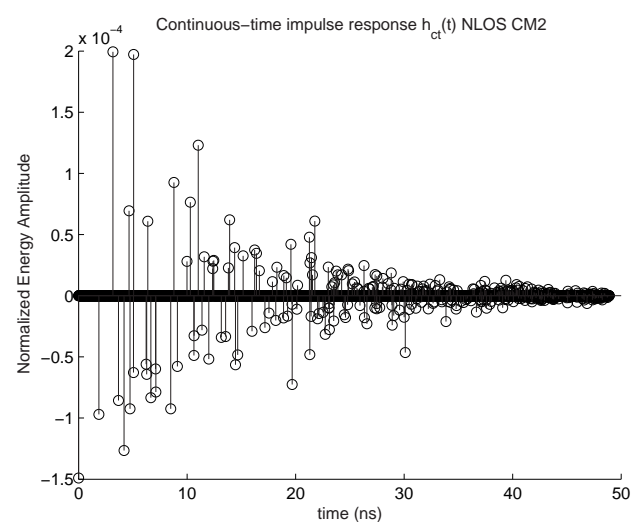

(a)

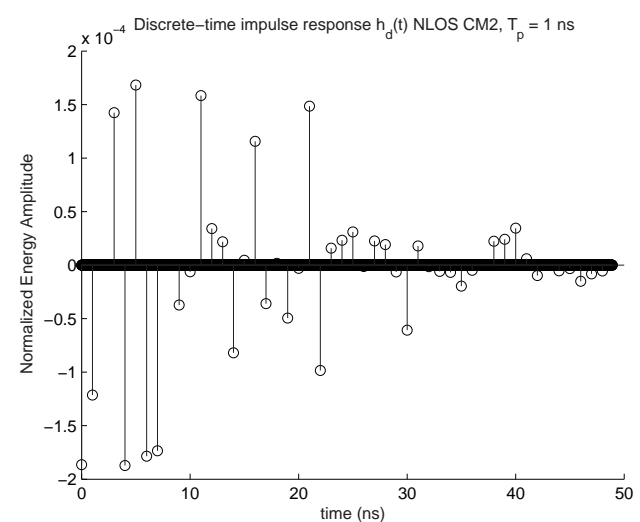

(b)

Figure 3.3: Fig. 3.3(a) illustrates a realization of the CM2 continuous-time impulse response. Fig. 3.3(b) is the equivalent discrete-time impulse response for the CM2 realization in Fig. 3.2(a) with bin width $T_{p}=1 \mathrm{~ns}$.

The mean excess delay $\overline{\tau_{i}}$ quantifies the time delay experienced by the mean of the multipath energy and is given by

$$
\overline{\tau_{i}}=\frac{\sum_{k} a_{k}^{2} \tau_{k}}{\sum_{k} a_{k}^{2}} .
$$

The rms delay spread or the standard deviation of the multipath energy is expressed as

$$
\sigma_{\overline{\tau_{i}}}=\sqrt{\overline{\tau_{i}^{2}}-\left(\overline{\tau_{i}}\right)^{2}}
$$

where

$$
\overline{\tau_{i}^{2}}=\frac{\sum_{k} a_{k}^{2} \tau_{k}^{2}}{\sum_{k} a_{k}^{2}} .
$$

Both parameters can be computed from a power delay profile (PDP) created from the temporal or spatial average of consecutive impulse response measurements or by averaging over a collection of single-realization parameters. We use computer simulation to compute the mean excess delay and rms delay spread directly from each impulse response realization using Equations 3.16 and 3.17 respectively and then averaging over the ensemble of channels. In both Equations 3.16 and 3.17, $a_{k}$ and $\tau_{k}$ refer to the $k^{\text {th }}$ discrete-time impulse response component and its arrival time respectively in the $i^{t h}$ impulse response realization. 
The remaining previously mentioned statistical multipath parameters, specifically $\mathrm{NP}_{10 \mathrm{~dB}}, \mathrm{NP}_{85}, E\left[\varepsilon_{\text {channel }}\right]$, and $\mathrm{E}[\mathrm{NP}]$ are evaluated similarly to $\overline{\tau_{i}}$ by averaging over the ensemble of simulated channel realizations. The results presented here are motivated by [8] as an attempt to quantitatively characterize the multipath channel upon which all results given in Chapter 6 are based. The effects of the channel modifications described in Section 3.2.4 can be seen in the parameter values as they deviate from those presented in $[8,9]$. Specifically, the increased bin width from $T_{p}=0.167 \mathrm{~ns}$ to $T_{p}=1 \mathrm{~ns}$ decreases the average number of components per realization as does truncation. The filtering of small MPCs with energy below $25 \mathrm{~dB}$ of the peak energy component decreases the average number of paths that capture 85 percent of the channel energy as well as the number of paths within $10 \mathrm{~dB}$ of the strongest path. We present the channel simulated multipath channel parameters in Tables 3.5 and 3.6 which correspond to a Tx-Rx separation distance of 1 and 2 meters respectively.

Table 3.5: Statistical multipath characterization parameters for the discrete-time impulse response with bin width $T_{p}=1 \mathrm{~ns}$ and Tx-Rx separation of $1 \mathrm{~m}$.

\begin{tabular}{||l|l|l|l|l||}
\hline \hline Channel Parameters & CM 1 & CM 2 & CM 3 & CM 4 \\
\hline $\mathrm{E}\left[\bar{\tau}_{i}\right]($ nsec) & 6.99 & 7.26 & 17.79 & 32.22 \\
\hline $\mathrm{E}\left[\sigma_{\bar{\tau}_{i}}\right](\mathrm{nsec})$ & 6.14 & 5.78 & 13.62 & 22.89 \\
\hline $\mathrm{NP}_{10 \mathrm{~dB}}$ & 7.66 & 7.39 & 14.31 & 22.19 \\
\hline $\mathrm{NP}_{85}$ & 3.51 & 3.42 & 5.51 & 7.82 \\
\hline $\mathrm{E}[\mathrm{NP}]$ & 27.57 & 23.70 & 60.74 & 103.76 \\
\hline $\mathrm{E}\left[\varepsilon_{\text {channel }}\right](\mathrm{dB})$ & -47 & -51 & -51 & -51 \\
\hline $\mathrm{E}\left[\alpha_{k}^{2}\right]\left(\mathrm{V}^{2}\right)$ & $7.24 E^{-7}$ & $3.35 E^{-7}$ & $1.31 E^{-7}$ & $7.67 E^{-8}$ \\
\hline $\mathrm{VAR}\left[\alpha_{k}^{2}\right]\left(\mathrm{V}^{2}\right)$ & $3.77 E^{-12}$ & $7.34 E^{-13}$ & $1.32 E^{-13}$ & $4.45 E^{-14}$ \\
\hline $\mathrm{E}\left[\left|\alpha_{k}\right|\right](\mathrm{V})$ & $5.75 E^{-4}$ & $3.99 E^{-4}$ & $2.45 E^{-4}$ & $1.88 E^{-4}$ \\
\hline
\end{tabular}

A chip-discrimination technique that discards chips with exceedingly large demodulated voltage metrics as presented in this thesis has a close relationship with the impulse response component tap coefficients $\alpha_{k}$. Each individual channel output MPC is scaled by the gain $\alpha_{k}$ of its particular arrival path according to Equation 5.1. The statistical properties of a channel model's tap coefficients $\alpha_{k}$ must be considered when choosing the simulation 
Table 3.6: Statistical multipath characterization parameters for the discrete-time impulse response with bin width $T_{p}=1 \mathrm{~ns}$ and Tx-Rx separation of $2 \mathrm{~m}$.

\begin{tabular}{||l|l|l|l|l||}
\hline \hline Channel Parameters & CM 1 & CM 2 & CM 3 & CM 4 \\
\hline $\mathrm{E}\left[\bar{\tau}_{i}\right]($ nsec) & 6.99 & 7.26 & 17.79 & 32.21 \\
\hline $\mathrm{E}\left[\sigma_{\bar{\tau}_{i}}\right](\mathrm{nsec})$ & 6.13 & 5.79 & 13.62 & 22.88 \\
\hline $\mathrm{NP}_{10 d B}$ & 7.66 & 7.39 & 14.30 & 22.19 \\
\hline $\mathrm{NP}_{85}$ & 3.51 & 3.42 & 5.51 & 7.82 \\
\hline $\mathrm{E}[\mathrm{NP}]$ & 27.56 & 23.71 & 60.73 & 103.72 \\
\hline $\mathrm{E}\left[\varepsilon_{\text {channel }}\right](\mathrm{dB})$ & -52.11 & -61.53 & -61.53 & -61.53 \\
\hline $\mathrm{E}\left[\alpha_{k}^{2}\right]\left(\mathrm{V}^{2}\right)$ & $2.23 E^{-7}$ & $2.96 E^{-8}$ & $1.16 E^{-8}$ & $6.77 E^{-9}$ \\
\hline $\mathrm{VAR}\left[\alpha_{k}^{2}\right]\left(\mathrm{V}^{2}\right)$ & $3.58 E^{-13}$ & $5.75 E^{-15}$ & $1.03 E^{-15}$ & $3.47 E^{-16}$ \\
\hline $\mathrm{E}\left[\left|\alpha_{k}\right|\right](\mathrm{V})$ & $3.19 E^{-4}$ & $1.19 E^{-4}$ & $7.28 E^{-5}$ & $5.60 E^{-5}$ \\
\hline
\end{tabular}

environment model; excessively large $\alpha_{k}$ of the desired user's channel biases the diversity gain and improves system performance while larger $\alpha_{k}$ in the co-located interferer channel magnify the effects of multi-user interference and system performance decreases. We quantify the statistical properties of the tap coefficients $\alpha_{k}$ through computer simulation of the following parameters: the mean squared value of $\alpha_{k}\left(E\left[\alpha_{k}^{2}\right]\right)$, the variance of $\alpha_{k}^{2}\left(\operatorname{VAR}\left[\alpha_{k}^{2}\right]\right)$, and the mean absolute value of $\alpha_{k}\left(\mathrm{E}\left[\left|\alpha_{k}\right|\right]\right)$. The parameters are included in Tables 3.5 and 3.6 with the other statistical multipath channel parameters at a Tx-Rx separation distance of 1 and 2 meters respectively.

\subsection{The RAKE Receiver}

The short pulse width $T_{p}$ of an IR transmitted chip produces natural temporal diversity that can be combined at the receiver to improve system performance. Indoor results have demonstrated that IR signals do not experience fading such that non-overlapping MPCs can be resolved individually [22]. The single correlator or matched filter demodulator discussed in Section 2.2 cannot capture each of the individual multipath components, which requires a bank of correlators called a RAKE receiver [23]. The RAKE receiver structure collects the time-shifted multipath replicas produced by the channel to improve system output SNR over the single matched filter [14]. Each correlator or finger demodulates a single time- 
shifted multipath component (MPC) and all finger outputs are linearly combined according to a variety of well-known techniques including Maximal Ratio Combining (MRC), Equal Gain Combining (EGC), and Minimum Mean Squared Error (MMSE) [5]. We adopt the MRC technique which weights the $k^{\text {th }}$ finger output $Z_{k}$ by the $k^{\text {th }}$ channel tap coefficient $\alpha_{k}$ before summing over all $L$ resolvable components. The maximal ratio combining technique which yields the maximum SNR at the RAKE receiver output for correctly estimated weight factors $\alpha_{k}$ is expressed mathematically as

$$
Z_{\text {chip }} \triangleq \sum_{n=0}^{L} \alpha_{n} Z_{n}
$$

The MRC voltage metric $Z_{c h i p}$ represents the contributions of the $L$ resolvable MPCs produced by a single transmitted chip in the $i^{\text {th }}$ channel realization. We base the bit estimate on the voltage metric $Z_{b i t}$ by summing the $N_{s}$ contiguously received chip metrics $Z_{\text {chip }}$ representing a single received bit [2]. In the optimal BPPM system, each RAKE finger is modeled as a single modified matched filter as described in Section 2.1 and the final bit estimation can be computed by comparing $Z_{b i t}$ to a zero threshold using the criteria given in Equation 2.8. Fig. 3.4 illustrates a BPPM MRC RAKE receiver structure for demodulating $L$ MPCs that arrive at time $\phi_{k}^{(0)}$ with energy proportional to $\alpha_{k}^{2}$. The max-

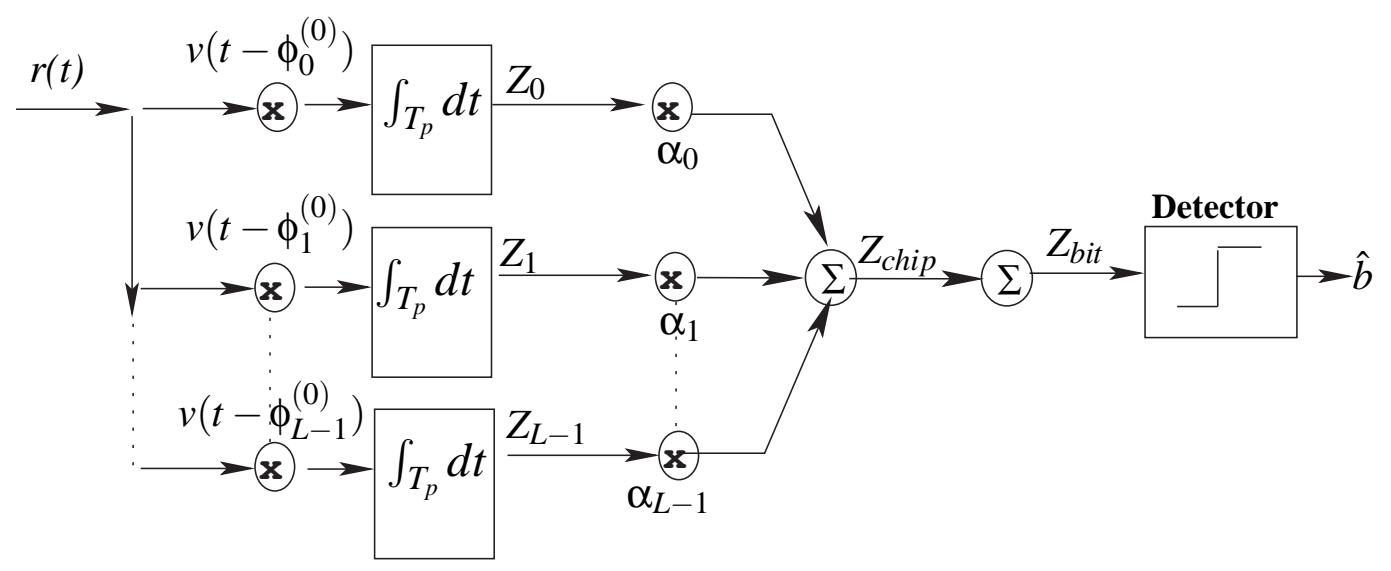

Figure 3.4: A block diagram of the $L$-finger RAKE receiver using MRC $[5,19]$.

imized output SNR of the MRC RAKE receiver requires estimation of the $k^{\text {th }}$ multipath component channel time delay $\tau_{k}$ and the $k^{\text {th }}$ tap coefficient $\alpha_{k}$. The $k^{\text {th }}$ received multipath 
component arrives at time $\phi_{k}^{(0)}=\tau_{k}+c_{j}^{(0)} T_{c}$, where $c_{j}^{(0)}$ is the pseudorandom time-hopping code of the desired user's $j^{\text {th }}$ transmitted chip and $\tau_{k}$ is the arrival time of the $k^{\text {th }}$ channel tap. In general $\tau_{k}$ and $\alpha_{k}$ can be determined by transmitting training sequences as in adaptive decision-feedback equalization, but this requires increased system complexity in terms of signal processing that is not considered in this work [18].

\subsubsection{RAKE Receiver Varieties}

In general the $i^{t h}$ channel realization produces $L=\frac{T_{m}^{i}}{T_{p}}$ resolvable multipath components (MPCs), where $T_{m}^{i}$ is the multipath spread of the $i^{t h}$ channel and $T_{p}$ is the channel time resolution [18]. A system that attempts to resolve all $L$ resolvable MPCs of the $i^{\text {th }}$ channel realization can be considered purely theoretical as in practice only a fraction of the $L$ resolvable paths are processed due to the strict timing requirements, power limitations, design complexity, and channel estimation of a UWB communications system [24]. We present three varieties of MRC RAKE receivers commonly found in literature to analyze system performance in multipath channels. Each RAKE variety is unique in the number of multipath components resolved and the criterion for choosing which components are resolved.

The ALL RAKE receiver (A-RAKE) is the theoretical case described above where all $L$ MPCs are resolved and combined to provide the maximum system performance of any RAKE variety. The A-RAKE receiver has unlimited resources in terms of signal correlators and instant adaptability such that matched filters identical to the set of received waveforms can be instantly obtained [25]. Although not implementable, this system serves as a theoretical benchmark that provides an upper limit of achievable performance [24]. The average channel energy gain $G$ can also be evaluated from the sum of ideally estimated tap weights averaged over the ensemble of channel realizations as given in Equation 3.12. This allows analytical calibration of the system based on the received energy output that is not possible with other RAKE varieties. 
The Selective RAKE receiver (S-RAKE) reduces the complexity of the A-RAKE receiver by processing only a subset $L_{s}$ of the $L$ resolvable MPCs. The S-RAKE receiver tracks all $L$ resolvable MPCs, but chooses to demodulate only the $L_{s}$ MPCs corresponding to the largest magnitude weighting coefficients $\alpha_{k}$ [24]. The most powerful received MPCs have the greatest chance of surviving channel and noise degradation to increase the combiner output SNR. As presented, the S-RAKE receiver can be considered a theoretical solution as tracking the $L$ MPCs is a complex operation in terms of system resources and still produces significant system latency. The added computation of analyzing or sorting the weighting coefficients undermines any savings provided by reducing the number of required correlators and combiner processing. An implementable S-RAKE receiver would estimate only a subset of the first arriving MPCs and choose to resolve paths corresponding to the largest $L_{s}$ magnitude weighting coefficients $\alpha_{k}$ from that subset.

The Partial receiver RAKE (P-RAKE) is the simplest and the only implementable RAKE variety that we present. Instead of analyzing the channel estimates as in the SRAKE receiver, the P-RAKE simply tracks only the first $L_{p}$ arriving MPCs regardless of each component's energy level. This technique reduces receiver complexity by removing the necessity to track all $L$ resolvable components as well as the removing the weighting coefficient analysis [24]. Of course the performance of the P-RAKE is inferior to the S-RAKE and A-RAKE performance, such that a tradeoff exists between receiver performance and complexity. The performance of the P-RAKE receiver is best in a LOS channel or a NLOS channel where the first arriving MPCs carry significant power [5]. The performance of the P-RAKE receiver is the most channel dependent of the receiver varieties that we present; the exponential PDP of the IEEE 802.15.3a channel model illustrated in Fig. 3.1 shows that acceptable performance should be expected of the P-RAKE variety in an IR system. 


\section{Chapter 4}

\section{Simulation Model}

We require a specific multi-user ad hoc network environment to analyze the efficacy of increased system performance by incorporating a chip-discrimination technique at each RAKE receiver finger output. The environment model that we present attempts to balance a possible mult-user network configuration exhibiting large near-far power ratio and the channel model limitations discussed in Section 3.2.3. We describe the environment model in Section 4.1 as well as specific system model assumptions and parameters in Sections 4.2.1 and 4.2.2 respectively.

\subsection{Model Environment}

We present a multi-user indoor ad hoc network configuration that satisfies the restrictions of the IEEE 802.15.3a channel model presented in Chapter 3. We create a pulselimited simulation environment illustrated in Fig. 4.1, where a desired transceiver node $T_{x}$ is located $2 \mathrm{~m}$ from its desired receiver $R_{x}$. The desired receiver node is surrounded by high-powered, co-located, interferer nodes at a distance of $2 \mathrm{~m}$. The channel through which the desired user and co-located interferers transmit is chosen based on the statistical channel parameters listed in Table 3.6 as we are unaware of any previous works that describe a 


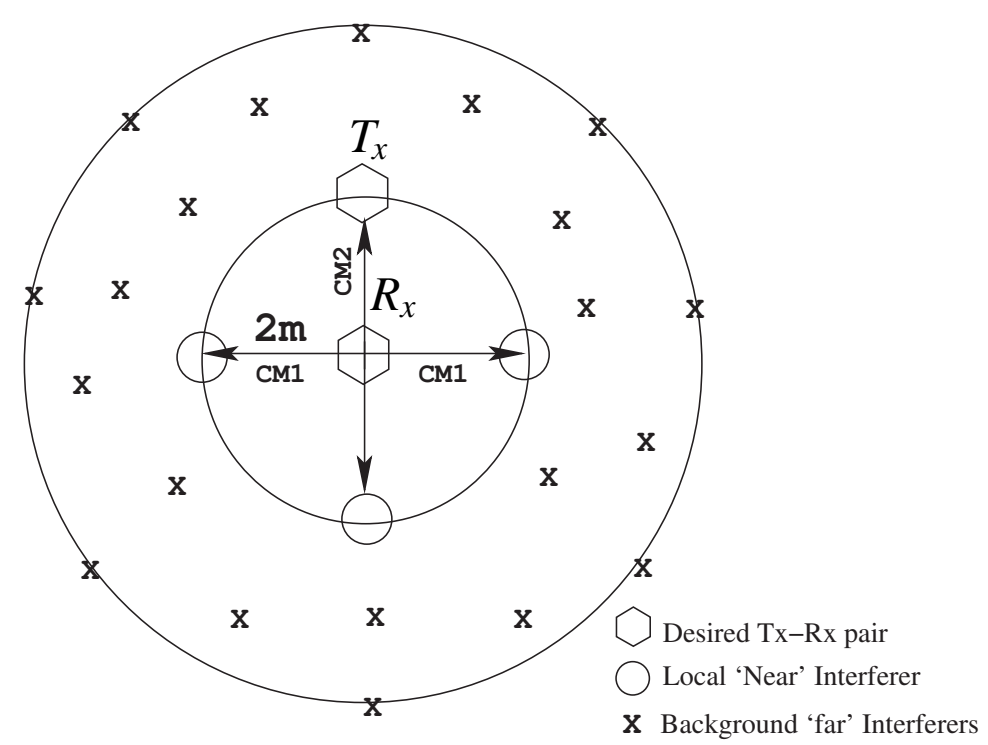

Figure 4.1: Diagram of the multi-user ad hoc network simulation environment.

particular multi-user network model using the IEEE 802.15.3a channel model.

The average channel energy gain $G$ determined in Equation 3.12 depends only on the Tx-Rx separation distance and the path loss exponent; hence all non line-of-sight (NLOS) channels have equal average energy gain $G$. By modeling the co-located interferer channel with CM1 and the desired channel with CM2, the large magnitude channel tap coefficients $\alpha_{k}$ of CM1 enhance the detrimental effects of the high-powered transmitted pulses that overwhelm the combined receiver output. We choose CM2 to model the desired channel based on the mean number of resolvable paths E[NP] and the mean-squared tap coefficient value $\mathrm{E}\left[\alpha_{k}^{2}\right]$. Fewer paths of arrival decrease the system diversity gain, fading, and computational complexity, while increasing the opportunity for multi-user MPC collisions; however, CM2 also produces tap coefficients $\alpha_{k}$ with the largest mean-squared value of the NLOS channel models. A simulation model that uses CM2 for the desired channel and CM1 for the co-located interferer channel augments the effects of multi-user interference while keeping the mean-squared values of the the tap coefficients as close in magnitude as possible.

A single channel realization from CM2 and CM1 are chosen for the desired user and 
$N_{N e a r}$ co-located interferers respectively every new transmitted data packet. An unspecified background user density exists with transmit power equal to that of the desired user. We model the performance degradation of the combined background user and thermal noise contribution as a zero-mean Gaussian process with variance $\sigma_{b g}^{2}$ determined from the mean bit energy $\overline{E_{b}}$ at the A-RAKE receiver combiner output. In the case of maximal ratio combining (MRC), $\overline{E_{b}}$ is given by

$$
\overline{E_{b}}=G^{2} N_{s}^{2} E_{R X}(1-R(\delta))^{2} .
$$

We calibrate the effects of the average equal-power background user density and thermal noise contributions such that an average background SNR, $\overline{S N R_{b g}}$, is maintained by solving

$$
\overline{S N R_{b g}}=\frac{\overline{E_{b}}}{\sigma_{b g}^{2}} .
$$

We include a block diagram of the simulated system in Fig. 4.2. The desired user $s_{0}(t)$ and $k^{\text {th }}$ interfering user $s_{k}(t)$ transmit through the $i^{\text {th }}$ channel realization of the IEEE 802.15.3a NLOS channel model CM2 and the LOS CM1 respectively. The desired RAKE receiver represents one of the RAKE varieties presented in Section 3.3.1: A-RAKE, S-RAKE, or P-RAKE. The term $n(t)$ represents zero-mean AWGN with variance $\sigma_{b g}^{2}$ that models the contributions of equal-powered distant interferers and thermal noise at the receiver; $n(t)$ is superimposed on the noiseless receiver output using the quasi-analytical method [26].

\subsection{System Model}

We create the simulated system model based on the fundamental impulse radio (IR) system assumptions first given in [3, 4] and the channel model limitations discussed in Section 3.2.3. As shown in Section 3.2.5, the channel assumptions directly impact the attained results and must be clearly presented. Section 4.2.1 lists the most important system 


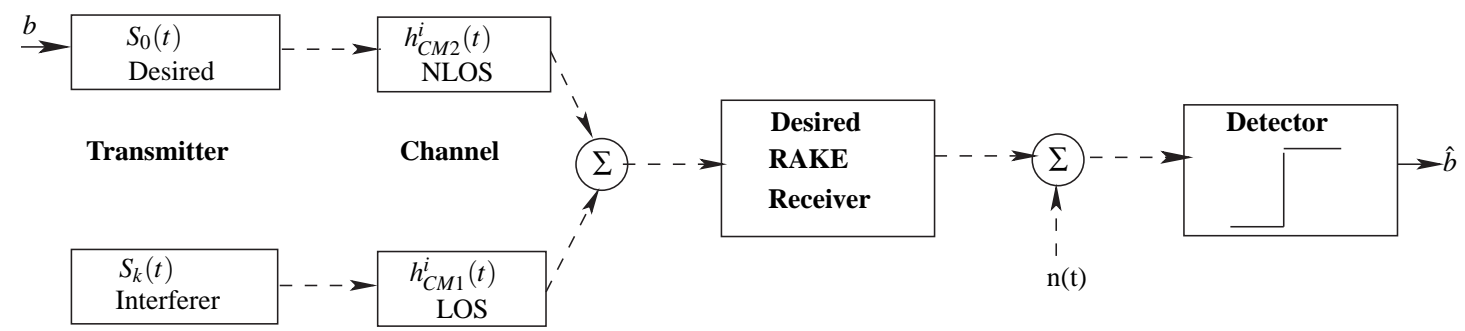

Figure 4.2: Block diagram of the impulse radio simulation model used to evaluate the effectiveness of the chip-discrimination technique applied to a possible multi-user ad hoc network configuration in the presence of multipath.

assumptions beginning with the basic impulse radio system assumptions and continuing to the more specific channel model assumptions. After presenting the underlying simulation model assumptions, Section 4.2.2 presents a table containing the exact simulation parameters used to achieve the results given in Chapter 6 within the constraints of the Section 4.2.1 assumptions.

\subsubsection{Assumptions}

The basic impulse radio system reported in [3,4] as well as the IEEE 802.15.3a channel model limitations described in $[8,9]$ influenced many assumptions concerning the simulated system. Unaware of previous multi-user impulse radio system investigations in the IEEE 802.15.3a multipath environment, we restricted simulation simplification through the assumptions listed below only by the validity of results.

\section{Transmitter / Receiver System Assumptions}

- An underlying assumption made for all work presented in this thesis is system linearity; the individual desired and interferer signals are linearly combined at the receiver during the demodulation process.

- All nodes of the analyzed IR network use consistent physical layer parameters; the frame width $T_{f}$, pulse width $T_{p}$, slot width $T_{c}$, and BPPM modulation shift $\delta$ are equivalent for all network users. 
- Equiprobable data bits are transmitted by all transceiver nodes in the multi-user impulse radio (IR) system.

- The propagation delay between the desired transmitter and receiver is known and without loss of generality is chosen to be $0 \mathrm{~ns}$.

- Perfect transmitter-receiver (Tx-Rx) clock synchronization allows all MPCs to be accurately tracked such that the received chip energy arrives within the designated correlation window.

- The variables $E_{T X}$ and $E_{R X}$ represent the desired transmitted and received energy respectively and are used interchangeably as all signal attenuation is accounted for by the channel impulse response $h(t)$.

- The width of the transmitted frame $T_{f}$ is chosen large enough such that inter-symbol interference (ISI) signal degradation is basically removed and the channel excess delay is usually exceeded.

\section{Channel Model Assumptions}

- The IEEE 802.15.3a channel is assumed to have a coherence time of a single data packet such that all received chips modulated by the bits composing a packet are correlated. Because each new channel impulse response realization is statistically independent from the previous realization, there is no correlation between received packets as explained in Section 3.2.3.

- A single channel model realization is used for all interfering users. Hence each of the desired user's received packets are statistically independent from the interferer packets, but all of the received interferer packets are correlated.

- The RAKE receiver fingers are considered to be uncorrelated such that perfect channel estimation of the desired user's channel exists. The channel estimation is performed independently for each impulse response component where the $n^{\text {th }}$ desired impulse response component has arrival time $\tau_{n}$ and real tap coefficient $\alpha_{n}$. We account for estimation error of channel tap coefficients $\alpha_{n}$ in Section 6.4. 
- Following from the ideal assumption of no propagation delay, the location of each received MPC is given by $\phi_{k}^{(n)}=\tau_{k}+c^{(n)} T_{c}$; $\tau_{k}$ represents the location of the $k^{\text {th }}$ MPC of the impulse response $h(t)$ and $c^{(n)} T_{c}$ is the transmission slot selected by the $n^{\text {th }}$ user's time-hopping (TH) code.

- The ad hoc network transceiver nodes move slowly and hold a constant distance of $2 \mathrm{~m}$ from the desired receiver. Otherwise the average channel energy gain $G$ must change for all Tx-Rx distances reached.

\subsubsection{Simulation Parameters}

The simulation system parameters are chosen to satisfy the assumptions listed above in Section 4.2.1. We present the simulation parameter values in Table 4.1 that produced the results presented in Chapter 6. We clearly state the only deviation from these parameters when investigating system sensitivity by varying a given parameter over a range of interest. The channel model parameter values given in Table 3.3 are not repeated in Table 4.1. 
Table 4.1: The system model simulation parameters used to produce the specific results presented in Chapter 6 .

\begin{tabular}{||l|c||}
\hline \hline Parameter & Value \\
\hline$N_{N e a r}$ (Number of Near Users) & 3 \\
\hline$N_{u}=N_{N e a r}+1$ (Total Number of Users) & 4 \\
\hline Desired Distance [m] (Desired Tx-Rx Separation) & 2 \\
\hline$S N R_{b g}[\mathrm{~dB}]$ (Average Background SNR) & 34 \\
\hline$N_{S}$ (Number Chips/Bit) & 100 \\
\hline Interferer Distance [m] (Interferer Tx-Rx Separation) & 2 \\
\hline$T_{f}[\mathrm{~ns}]$ (Transmit Frame Width) & 102 \\
\hline$E_{T X}[\mathrm{~dB}]$ (Desired Energy/Chip) & 0 \\
\hline$(I / S)_{T X}[\mathrm{~dB}]$ & \\
$($ Co-located Interferer-to-Desired Signal Ratio at Transmitter) & 40 \\
\hline$T_{p}$ [ns] (Transmit Pulse Width) & 1 \\
\hline Desired CM (Desired Channel Model) & $\mathrm{CM} 2$ \\
\hline Interferer CM (Interferer Channel Model) & $\mathrm{CM} 1$ \\
\hline packet [bits] (Number of Bits/Packet) & 100 \\
\hline dt [ns] (Sample Time) & 0.01 \\
\hline$L_{S}$ (Number of S-RAKE Fingers) & 4 \\
\hline$L_{p}$ (Number of P-RAKE Fingers) & 4 \\
\hline$\tau_{m f}$ (Correlation Window Width) [ns] & 1.23 \\
\hline$\delta$ (BPPM Shift) [ns] & 0.212 \\
\hline
\end{tabular}




\section{Chapter 5}

\section{Multi-User Multipath System Model}

\section{with Chip Discrimination}

In a large near-far power ratio environment, the received co-located interferer MPCs arrive with power levels much greater than the desired user. A collision occurring between a desired and interferer MPC often overwhelms the combined chip metric $Z_{\text {chip }}$ and eventually the bit metric $Z_{b i t}$ upon which bit decisions are based. To mitigate the effects of strong interferer collisions on system performance, we extend the work presented in [1] and briefly described in Section 2.2 by applying the basic technique of chip discrimination to a multipath channel. We begin this chapter by providing mathematical expressions in Section 5.1 for the received signal present at the RAKE combiner under the assumption of a linear system. Section 5.2 describes the autonomous chip-discrimination technique as applied to a multipath environment and proposes a novel chip discriminator to be applied to each RAKE receiver finger output $Z_{k}$. 


\subsection{Received Signal}

The $n^{\text {th }}$ user's received channel output $r^{(n)}(t)$ is expressed generically as the convolution of the $n^{\text {th }}$ user's transmitted signal $s^{(n)}(t)$ from Equation 2.5 and the $i^{\text {th }}$ discrete-time channel impulse response realization $h_{i}(t)$ from Equation 3.14 by

$$
r^{(n)}(t)=s^{(n)}(t) * h_{i}(t)
$$

We expand the $n^{t h}$ user's received signal $r^{(n)}(t)$ as the sum of all received multipath components (MPCs) produced by the $i^{t h}$ impulse response realization $h_{i}(t)$ for a single transmitted bit expressed as

$$
r^{(n)}(t)=\sqrt{E_{r x}^{(n)}} \sum_{j=0}^{N_{s}-1} \sum_{k=0}^{L-1} \alpha_{k}^{i} w\left(t-j T_{f}-\delta d_{\left\lfloor j / N_{s}\right\rfloor}^{(n)}-\phi_{k}^{(n)}\right)+n(t) .
$$

The term $n(t)$ represents the equal-powered, multi-user background interference and thermal noise degradation modeled as Gaussian to satisfy Equation 4.2. The total received signal $r(t)$ is given by

$$
r(t)=\sum_{n=0}^{N_{u}-1} \sqrt{E_{r x}^{(n)}} \sum_{j=0}^{N_{s}-1} \sum_{k=0}^{L-1} \alpha_{k}^{i} w\left(t-j T_{f}-\delta d_{\left\lfloor j / N_{s}\right\rfloor}^{(n)}-\phi_{k}^{(n)}\right)+n(t)
$$

where the contributions of $N_{N e a r}$ co-located interferers are linearly combined with the desired signal at the receiving antenna.

\subsection{Chip Discrimination}

The opportunity for high-powered co-located interferers to degrade system performance in the Gaussian channel occurs only during $\tau_{m f}$ ns of every received frame of duration $T_{f} \mathrm{~ns}$, when the receiver's correlation window opens to collect received energy. The probability 
of avoiding a chip collision when $N_{N e a r}$ high-powered co-located interferers are present is given by

$$
p_{a c}=\left(1-\frac{\tau_{m f}}{T_{f}}\right)^{N_{\text {Near }}} \times\left(1-\frac{T_{p}}{T_{f}}\right)^{N_{\text {Near }}}
$$

where $\tau_{m f}$ is the width of the receiver correlation window. All received energy is ignored by the receiver unless it arrives inside of the $\tau_{m f}$ ns interval beginning at a pseudorandom frame location indicated by the desired user's time-hopping (TH) code [1]. In the case of the multipath channel, each RAKE finger independently tracks and demodulates one of $L_{d}$ individual resolvable multipath components (MPCs) produced by each desired transmitted chip. The probability of avoiding a collision with any of the $L_{i}$ MPCs produced by each of the $N_{\text {Near }}$ co-located interferers is given by

$$
p_{a c}=\left(1-\frac{L \tau_{m f}}{T_{d}}\right)^{N_{N e a r}} \times\left(1-\frac{T_{p} L_{i}}{T_{i}}\right)^{N_{\text {Near }}}
$$

for the discrete-time system model introduced in Chapter 4. In Equation 5.5, $L$ is the number of receiver RAKE fingers, $T_{d}$ is the duration of the received desired signal, and $T_{i}$ is the duration of the received interferer signal.

The Gaussian channel expression in Equation 5.4 can be evaluated directly, unlike Equation 5.5 which should be averaged over an ensemble of channel realizations. When considering the A-RAKE receiver, $L$ is replaced by $L_{d}$, the total number of RAKE fingers necessary to resolve all multipath components produced by a single transmitted chip. The probability of avoiding a chip collision with a co-located user can be equivalently evaluated for the P-RAKE receiver by substituting $L_{p}$ for $L$. Equation 5.5 cannot be evaluated for the S-RAKE receiver because the probability of avoiding a chip collision becomes conditioned on the particular $L_{S}$ RAKE fingers used for signal demodulation. Although the BER performance of A-RAKE is superior to S-RAKE and P-RAKE, the larger number of RAKE fingers make the system more susceptible to strong co-located chip collisions. In fact, P-RAKE exhibits the highest probability of avoiding a collision followed closely by the S-RAKE and finally the A-RAKE. 
In general there exist $L_{i}$ opportunities per transmitted interferer chip for a single interfering MPC collision to corrupt the received bit estimate metric $Z_{b i t}$ in the multipath channel. To mitigate the effects of such collisions, we propose applying the autonomous chipdiscrimination technique at the output of each RAKE finger $Z_{k}$ as illustrated in Fig. 5.1. This method is simply an increase in complexity to the maximal ratio combining (MRC)

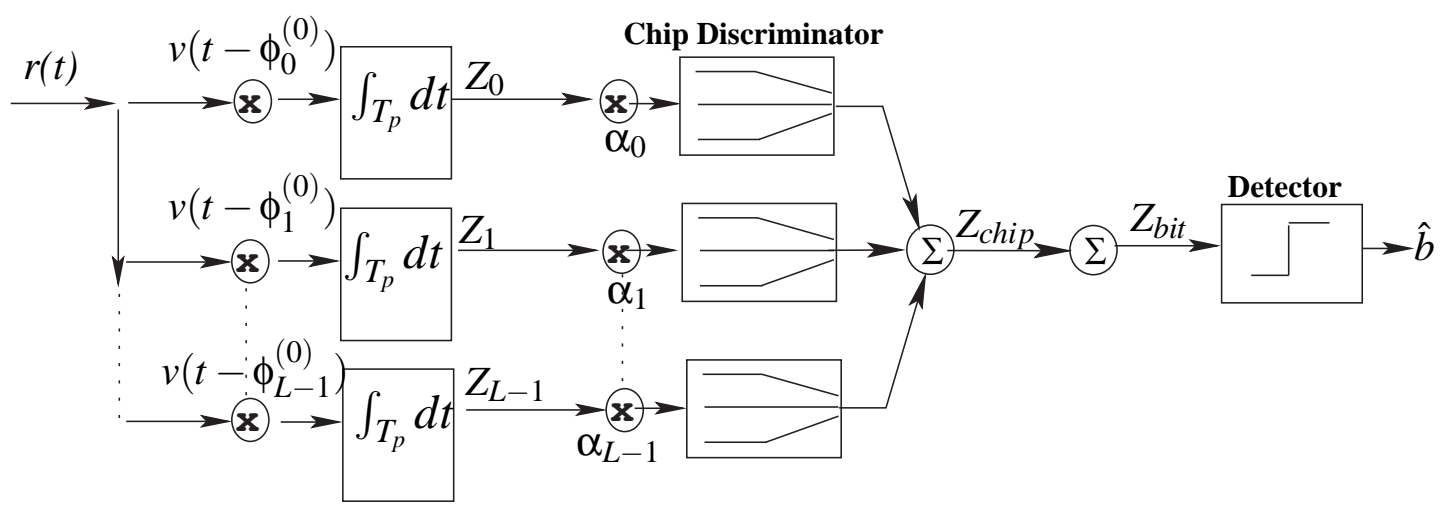

Figure 5.1: A block diagram of the $L$-finger RAKE receiver using MRC with the chip discriminator of Fig.5.2 applied to each weighted finger output [5, 19].

RAKE receiver of Fig. 3.4, where the novel chip discriminator device given in Fig. 5.2 restricts demodulated MPCs that exceed a certain threshold from contributing to the bit estimate metric $Z_{b i t}$. The chip-discrimination technique requires estimation of the channel

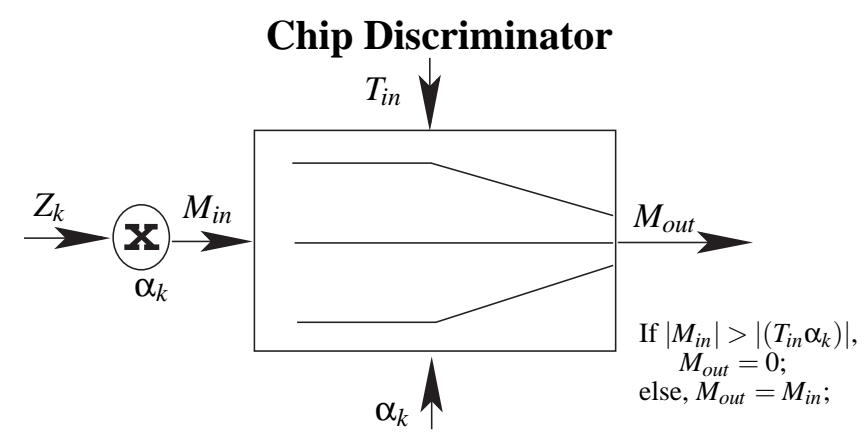

Figure 5.2: A chip discriminator applied to each RAKE receiver finger output $Z_{k}$ removes the weighted voltage metrics $M_{\text {in }}$ that exceed a specified threshold $\left|\left(T_{i n} \alpha_{k}\right)\right|$ in magnitude.

coefficients $\alpha_{k}$, but this is already a fundamental requirement when implementing RAKE receivers that use MRC to combine diversity. With no coordination of network nodes, the 
technique exploits the channel estimations $\alpha_{k}$ and $\tau_{k}$ to create a dynamic threshold proportional to each individual MPC.

In the Gaussian channel investigated in [1], the signal attenuation is considered constant for both the desired user and co-located interferers; all signal degradation is attributed to the multi-user interference and added noise. The lack of random attenuation allows a constant threshold $X$ from Equation 2.6 to be chosen such that the magnitude of all demodulated metrics is compared to $|X|$ before it contributes to the bit estimate metric $m_{b i t}$. A constant threshold $X$ does not exist for the multipath channel analyzed in this work, where the MPC signal attenuation imposed by each channel coefficient $\alpha_{k}$ varies over many orders of magnitude. We combat the large variance of received voltage levels by defining a dynamic threshold that consists of a deterministic threshold value $T_{\text {in }}$ weighted by the channel coefficient $\alpha_{k}$ such that the result is proportional to the voltage level of each individual demodulated MPC. A deterministic threshold value $T_{\text {in }}$ equivalent to $X$ with values specific to the multipath channel of interest is chosen and then multiplied by $\alpha_{k}$ to create the threshold to which the magnitude of the RAKE finger outputs $\left|Z_{k}\right|$ is compared.

As seen in Fig. 5.2, each RAKE finger output $Z_{k}$ is weighted by the estimated channel tap coefficient $\alpha_{k}$ to create the chip discriminator input metric $M_{\text {in }}$ as in ordinary maximal ratio combining; the resulting magnitude of $M_{\text {in }}$ is then compared to the calculated threshold level according to

$$
M_{\text {out }}=\left\{\begin{array}{ll}
0 & \text { for }\left|M_{\text {in }}\right|>\left|\left(T_{i n} \alpha_{k}\right)\right| \\
M_{\text {in }} & \text { for }\left|M_{i n}\right| \leq\left|\left(T_{i n} \alpha_{k}\right)\right|
\end{array} .\right.
$$

We define the deterministic threshold $T_{i n}$ with reference to the magnitude of the ideal matched filter output voltage sample. This makes $T_{i n}$ system specific as it depends on the desired user transmit power and receiver timing accuracy such that the maximum amount of desired user's energy is captured by each RAKE finger. The total chip discriminator threshold $\left|\left(T_{i n} \alpha_{k}\right)\right|$ adapts to the channel to which it is applied through the channel tap co- 
efficients $\alpha_{k}$. The demodulated finger voltage metrics $M_{i n}$ whose magnitude exceed the threshold $\left|\left(T_{i n} \alpha_{k}\right)\right|$ are assumed to be corrupted by an interferer collision and are discarded such that $M_{\text {out }}$ becomes zero and contributes nothing to the current bit estimate $Z_{\text {bit }}$. If $\left|M_{i n}\right|$ is within the threshold boundary then we assume the finger output voltage to be uncorrupted and it is passed to the combiner unaltered. The weighted finger output metrics $M_{\text {out }}$ combine linearly over a single chip to produce the chip metric $Z_{c h i p}$ and over $N_{s}$ received chips to create the bit metric $Z_{b i t}$. The bit estimate $\hat{b}$ is computed based on $Z_{b i t}$ according to Equation 2.8 for a BPPM system. 


\section{Chapter 6}

\section{Results}

We examine the effectiveness of the chip-discrimination technique presented in Chapter 5 in the presence of the IEEE 802.15.3a channel model. This chapter presents the computer simulation results for a multi-user impulse radio (IR) system operating in the indoor environment described in Section 4.1 using the system parameters given in Table 4.1. We believe comparison of three RAKE varieties A-RAKE, S-RAKE, and P-RAKE to be the most beneficial presentation to illustrate the different performance characteristics of each receiver when chip discrimination is applied. The results presented in this chapter excluding Section 6.4 assume perfect channel estimation of tap delays $\tau_{k}$ and coefficients $\alpha_{k}$.

We begin in Section 6.1 by examining the system performance achieved at different threshold levels $T_{i n}$, where $T_{i n}$ is given in $\mathrm{dB}$ with reference to the magnitude of the ideal matched filter output voltage sample. We describe an optimal performance region of $T_{\text {in }}$ in terms of average BER specific to the system investigated as well as the consequences of incorrectly choosing $T_{i n}$. A major concern in choosing a performance-enhancing technique is the system's sensitivity to changes in system parameters. Section 6.2 analyzes the performance sensitivity in terms of achievable BER, to the system parameters $N_{s},(I / S)_{T X}$, and $N_{\text {Near }}$. We extend the parameter sensitivity investigation in Section 6.3 by analyzing the probability of discarding a chip at different thresholds $T_{i n}$ when the system parameters 
$N_{N e a r}$ and $(I / S)_{T X}$ are varied over an applicable range. Section 6.4 concludes the chapter by analyzing the average BER performance when imperfect channel estimation provides noisy channel tap coefficients $\alpha_{k}$. Although we group the results according to the specific experiments conducted, the influences of each parameter on system performance can be seen throughout the results presented; therefore explanations consistently draw from the different sections to reinforce conclusions.

\subsection{Threshold Performance}

An impulse radio (IR) system that incorporates chip discrimination at each RAKE receiver finger output $Z_{k}$ requires channel estimation to obtain the necessary channel tap coefficients $\alpha_{k}$. From Fig. 5.2 and Equation 5.6 we see that accurate evaluation of both $\alpha_{k}$ and $T_{i n}$ determines the threshold applied to each received MPC and hence the system performance attained. In this section we use simulation to find an optimal performance range of $T_{i n}$, in terms of average BER, using the system model given in Chapter 4 under the assumption of perfect channel estimation. As described in Section 5.2, $T_{\text {in }}$ is system specific; the objective is not to describe a method for obtaining an optimal threshold $T_{i n}$, but to demonstrate the achievable average BER performance of the chip-discrimination technique for varying $T_{\text {in }}$ levels.

We illustrate the average BER system performance in Fig. 6.1 for a range of $T_{\text {in }}$ from $-146 \mathrm{~dB}$ to $6 \mathrm{~dB}$. Many important facts concerning the multi-user IR system in a multipath channel become clear from reviewing Fig. 6.1. First, we can see three distinct performance curves corresponding to the three RAKE varieties: A-RAKE, S-RAKE, and P-RAKE. Fig. 6.1 illustrates the typical performance of these RAKE varieties where A-RAKE always achieves the best performance followed by S-RAKE and with P-RAKE achieving the poorest performance. When the number of S-RAKE and P-RAKE fingers, $L_{s}$ and $L_{p}$ respectively, are increased, performance approaches the A-RAKE benchmark where $L$ fingers are employed. In this particular case, there is nearly an order of magnitude separation 


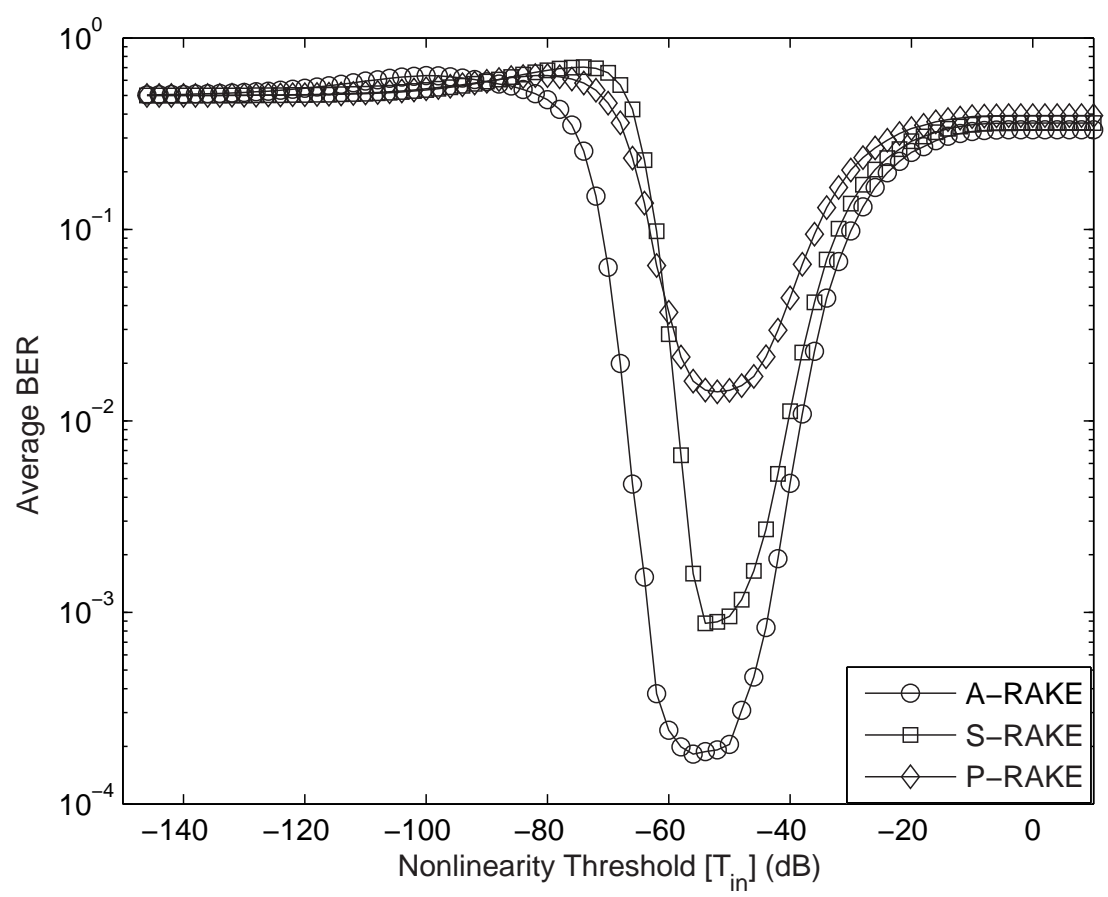

Figure 6.1: Average BER achieved for a chip discriminator threshold $T_{\text {in }}$ varying between $-146 \mathrm{~dB}$ and $6 \mathrm{~dB}$ with $N_{\text {Near }}=3$ and $(I / S)_{T X}=40 \mathrm{~dB}$.

in average BER for the three RAKE varieties at the optimal BER threshold $T_{\text {in }}=-56 \mathrm{~dB}$.

The general behavior of each simulated RAKE receiver variety is the same: the optimal $T_{\text {in }}$ in terms of the lowest BER occurs at approximately $-56 \mathrm{~dB}$, in the negative direction from $-56 \mathrm{~dB}$ the curves converge to 0.5 , and in the positive direction the curves converge to the average BER of an equivalent system without chip discrimination. The chip-discrimination technique is robust in the region between $-60 \mathrm{~dB}$ and $-46 \mathrm{~dB}$ that we label the "optimal threshold region" over which performance only slightly deviates from the optimal average BER achieved at $T_{i n}=-56 \mathrm{~dB}$. Outside of the optimal BER threshold region, average BER performance is greatly affected by slight deviations in the threshold $T_{i n}$. When $T_{\text {in }}$ is increased, too few chips corrupted by strong interferer collisions are discarded; alternatively, decreasing $T_{\text {in }}$ beyond the bounds of the optimal threshold region discards not only $Z_{k}$ resulting from strong interferer collisions, but also uncorrupted chips with greater certainty. 
If we increase $T_{\text {in }}$ beyond its optimal threshold value at $-56 \mathrm{~dB}$, average BER performance decays to that of an equivalent system with no chip discrimination. Fig. 6.8 shows that for the impulse radio system considered here with parameters given in Table 4.1, no chips are discarded when $T_{\text {in }}>-12 \mathrm{~dB}$. The threshold $T_{\text {in }}$ is so large that collisions resulting from interferers with transmit power $40 \mathrm{~dB}$ larger than the desired user are not discarded by any of the RAKE fingers. Chip discrimination cannot function when $T_{i n}$ is too large and the system operates as if no chip discriminators are applied to the RAKE finger outputs. The resulting chip metric $Z_{\text {chip }}$ contains contributions from all finger outputs $Z_{k}$, where a corrupted $Z_{k}$ with large magnitude corrupts $Z_{c h i p}$ and eventually overwhelms $Z_{b i t}$. In Fig. 6.1, increasing $T_{\text {in }}$ from $-56 \mathrm{~dB}$ to no chip discrimination at $-12 \mathrm{~dB}$ increases the average BER by approximately an order of magnitude every $14 \mathrm{~dB}$.

The average BER converges to the performance of an equivalent system without chip discrimination at $T_{\text {in }}=-12 \mathrm{~dB}$; further positive increases beyond $-12 \mathrm{~dB}$ have no effect on average BER. When no corrupted chips are discarded, the average BER with only three high-powered co-located interferers and $N_{s}=100$ is between 0.3 and 0.4 depending on the RAKE receiver variety. In fact when no chip discrimination is employed, the target average BER of $10^{-3}$ can never be achieved for a meaningful throughput $R_{S}$ since according to Equation 2.3 the throughput is inversely proportional to $N_{s}$. Fig. 6.2 demonstrates the average BER performance for an equivalent impulse radio system that employs no chip discrimination where $N_{s}$ ranges from 1 to 100. The most striking observation from Fig. 6.2 is the increased average BER performance achieved by the P-RAKE receiver compared to the S-RAKE and A-RAKE varieties for small $N_{s}$.

The A-RAKE receiver that usually out-performs the other RAKE varieties achieves the worst performance for small values of $N_{s}$. According to Equation 5.5, the probability of avoiding a co-located interferer collision is greater for the P-RAKE receiver because the duration of the open correlation window is much shorter than for the A-RAKE receiver; fewer interferer collisions occur when fewer MPCs are resolved. The P-RAKE average BER performance surpasses the S-RAKE performance because the S-RAKE chooses the 


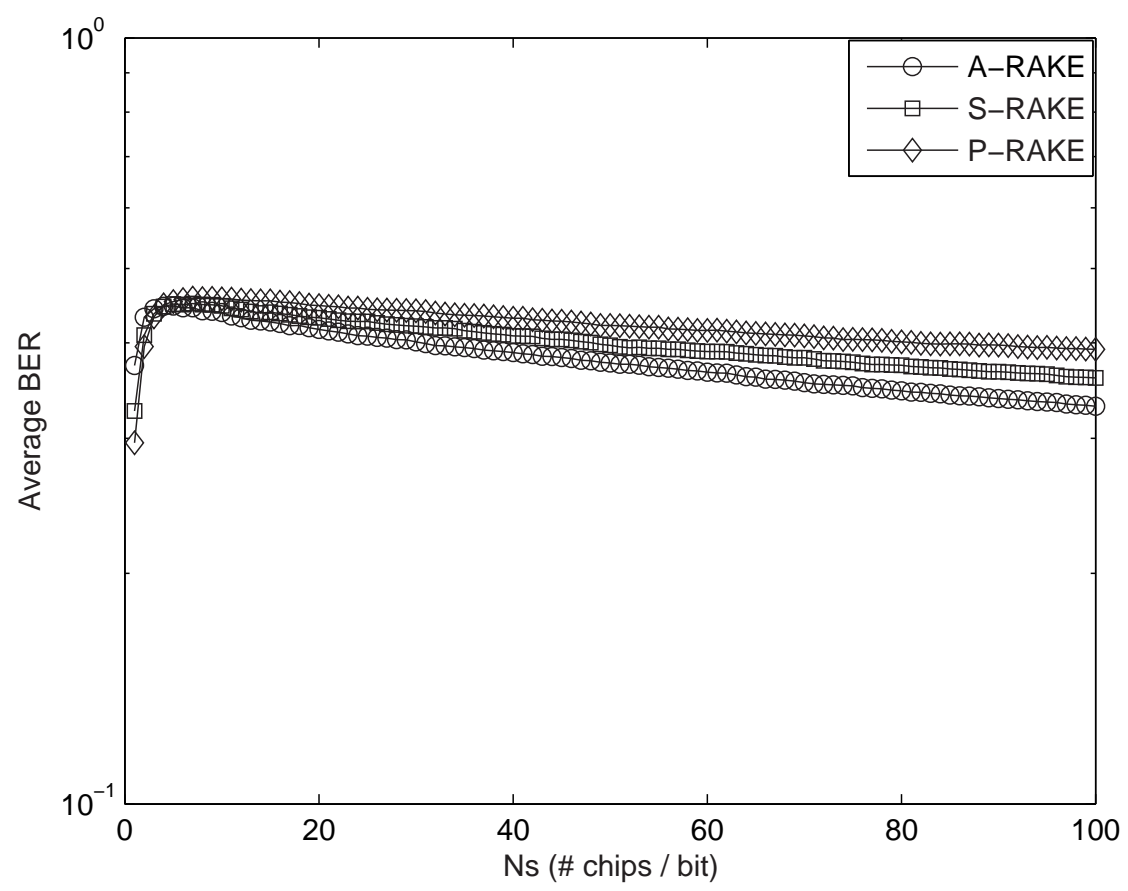

Figure 6.2: Average BER achieved for a multi-user IR system with no chip discrimination. The number of transmitted chips/bit $N_{S}$ is varied from 1 to 100 with $N_{\text {Near }}=3$ co-located interferers and $(I / S)_{T X}=40 \mathrm{~dB}$.

$L_{S}$ largest magnitude paths to resolve. Each S-RAKE resolved MPC is scaled by the largest channel tap coefficients $\alpha_{k}$ in the channel realization which enhances the detrimental effects of strong interferer collisions. The P-RAKE variety that receives the first $L_{p}$ resolvable components resolves many of the same components as S-RAKE because the channel is exponentially decreasing such that the first arrivals are usually the largest in magnitude; however, not all received components are scaled by the largest $\alpha_{k}$ of the realization which allows performance to exceed the S-RAKE for small spreading sequences $N_{s}$.

As $N_{s}$ increases, the average BER performance returns to the ordinary case where ARAKE achieves the best performance followed by S-RAKE and P-RAKE. The increased diversity gain and larger magnitude coefficients $\alpha_{k}$ that destroyed BER performance at small $N_{s}$ values enable the system to offset the effects of co-located interferer collisions with more reliability as $N_{s}$ increases. The probability of avoiding a co-located interferer collision is decreased, but the increased diversity gain from resolving all $L$ paths increases 
system performance. At $N_{s}=100$, the system performance is exactly as indicated in Fig. 6.1 for $T_{i n} \geq-12 \mathrm{~dB}$ such that no $Z_{k}$ corrupted by interferer collisions are discarded. Unlike the S-RAKE and P-RAKE receivers, the A-RAKE receiver exceeds its $N_{s}=1$ initial BER performance at $N_{s}=100$; however, the average BER performance for all RAKE varieties is still more than three orders of magnitude from its optimal value at $T_{i n}=-56 \mathrm{~dB}$ in the equivalent system employing chip discrimination.

The previously described consequence of choosing $T_{i n}$ too large is that the system regresses to an equivalent system with no chip discrimination; the consequences for choosing $T_{\text {in }}$ too small can be far worse. If we decrease $T_{\text {in }}$ beyond its optimal BER value at $-56 \mathrm{~dB}$, average BER performance decays quickly to 0.5 . As $T_{\text {in }}$ decreases, not only are the exceedingly large $Z_{k}$ discarded, but uncorrupted MPCs are discarded with increasing frequency. Fig. 6.7 illustrates that in the ideal case of no co-located interferers, reducing $T_{\text {in }}$ beyond $-56 \mathrm{~dB}$ achieves a nonzero probability of incorrectly discarding a chip. Because the S-RAKE receiver tracks and demodulates the largest magnitude $L_{S}$ MPCs, its average BER performance is always expected to surpass the P-RAKE variety; however, the performance of the S-RAKE receiver decays beyond the P-RAKE performance at $T_{\text {in }} \leq-62 \mathrm{~dB}$. The uncorrupted chips resolved by the S-RAKE more frequently exceed the threshold for $T_{\text {in }} \leq-56 \mathrm{~dB}$ as shown in Fig. 6.7 which forces the S-RAKE average BER to approach 0.5 as $T_{\text {in }}$ decreases beyond $-62 \mathrm{~dB}$.

There is a region of $T_{\text {in }}$ between approximately -68 and $-120 \mathrm{~dB}$ that we label the elevated BER region that actually allows co-located interference to overwhelm the bit estimate metric $Z_{b i t}$ such that the average BER surges above 0.5 . When $T_{\text {in }}$ decreases below the value where approximately $95 \%$ of chips are discarded shown in Fig. 6.7, partial collisions with highly attenuated interferer MPCs incorrectly biases the bit estimate metric $Z_{b i t}$. In the elevated BER region, the chip metric $Z_{\text {chip }}$ is calculated from approximately $5 \%$ of the resolved MPCs; this frequently leaves $Z_{\text {chip }}$ with a value of zero, especially for the $\mathrm{S}-\mathrm{RAKE}$ and P-RAKE receivers where the number of fingers $L_{s}$ and $L_{p}$ respectively are much fewer than $L$ as used by the A-RAKE. The resulting average BER becomes dominated by an abundance of decisions based on a bit metric $Z_{b i t}$ that is either zero or incorrect. 
The average BER with the S-RAKE receiver first exceeds 0.5 at $T_{\text {in }} \approx-68 \mathrm{~dB}$ and remains above 0.5 until $T_{\text {in }} \approx-100 \mathrm{~dB}$. The large magnitude MPCs resolved by the S-RAKE receiver are discarded in the elevated BER region unless partial collisions bring $\left|Z_{k}\right|$ below the calculated threshold. The P-RAKE and A-RAKE also have elevated BER regions, but they come at decreasingly smaller $T_{i n}$ because the small magnitude $\left|Z_{k}\right|$ resolved by these RAKE varieties require a small $T_{i n}$ to be discarded. The elevated BER regions for the P-RAKE and A-RAKE receivers begins and ends at $T_{\text {in }} \approx-70$ to $-90 \mathrm{~dB}$ and $T_{\text {in }} \approx-90$ to $-120 \mathrm{~dB}$ respectively. When $T_{\text {in }}$ decreases below $-120 \mathrm{~dB}$, all chips are discarded and $Z_{b i t}$ becomes zero which gives the detector an equiprobable bit estimate of 0.5 regardless of the transmitted data bit or RAKE receiver variety.

\subsection{System Performance Sensitivity}

The sensitivity of an impulse radio (IR) system employing chip discrimination to basic system parameters is of great importance when determining the optimal range of the threshold $T_{i n}$ for a given system. To examine the system performance for a variety of IR system configurations, we varied the system parameters $(I / S)_{T X}, N_{S}$, and $N_{N e a r}$ over a range of possible values. The results given in this section demonstrate that the system BER performance illustrated in Fig. 6.1 is not unique to a single set of parameters, but is in fact characteristic of many practical system configurations. We found no optimal threshold $T_{\text {in }}$ that consistently produced the best average BER performance for all varying parameters; however, we found that the optimal threshold $T_{i n}$ for all experiments remained within the optimal threshold region defined in Section 6.1 with reference to the results illustrated in Fig. 6.1.

We begin by investigating system performance in terms of average BER for different co-located interferer transmitter power levels which are adjusted through the transmitter Interferer-to-Signal ratio $(I / S)_{T X}$. We simulated the system using the parameters listed in 
Table 4.1, with the exception of $(I / S)_{T X}$ which varys from 0 to $50 \mathrm{~dB}$. We include the results for the A-RAKE receiver only in Fig. 6.3 since all curves exhibit the same behavior and differ only in the specific performance values achieved. The intention of Fig. 6.3 is to

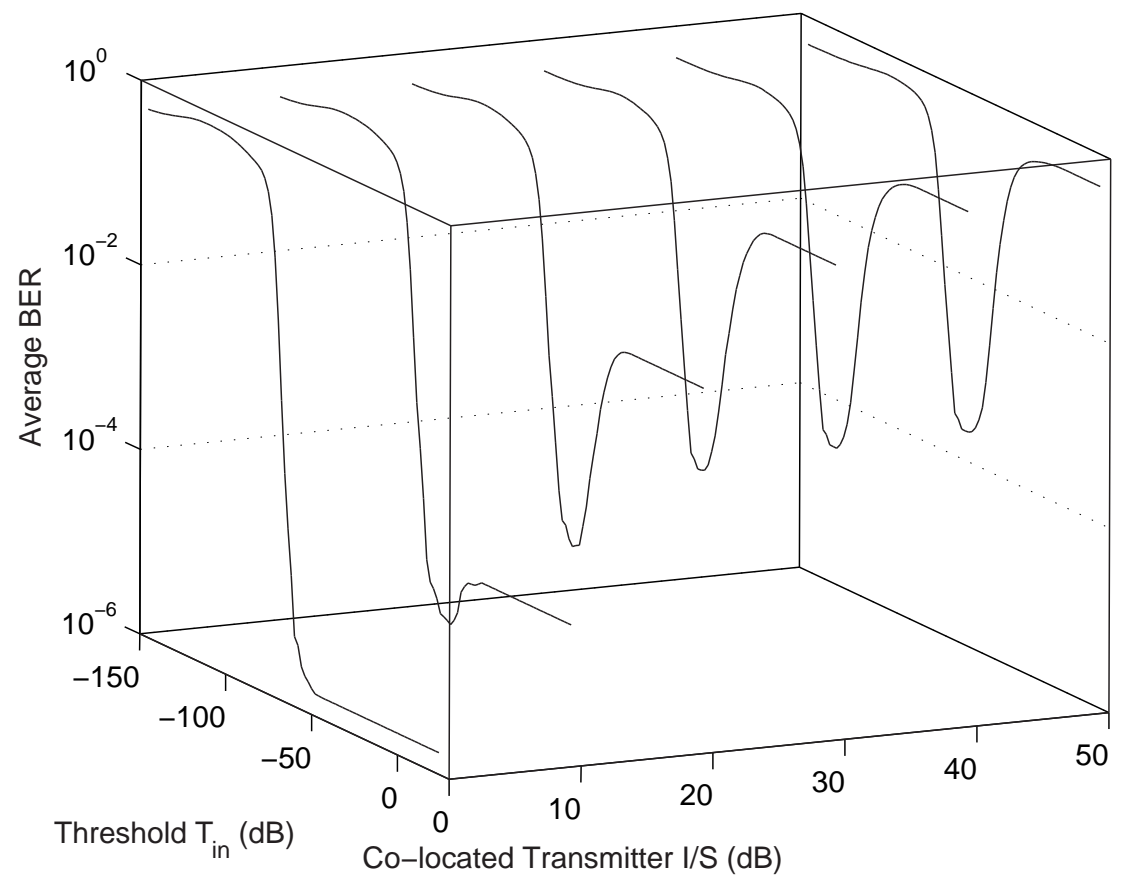

Figure 6.3: A-RAKE 3-D plot of average BER for a varying threshold $T_{\text {in }}$ from $-150 \mathrm{~dB}$ to $10 \mathrm{~dB}$. There are 3 co-located interferers with $(I / S)_{T X}$ ranging from $0 \mathrm{~dB}$ to $50 \mathrm{~dB}$.

demonstrate the basic effect of $T_{\text {in }}$ over the range of -150 to $10 \mathrm{~dB}$ on the average BER for different co-located interferer power levels. Specific performance values can be seen in Fig. 6.1 which is equivalent to the curve of Fig. 6.3 at $(I / S)_{T X}=40 \mathrm{~dB}$.

The average BER curves in Fig. 6.3 clearly illustrate the increased effectiveness of the chip discrimination technique in large near-far power ratio environments. The leftmost curve which corresponds to $(I / S)_{T X}=0 \mathrm{~dB}$ demonstrates that no additional performance is gained by applying the chip discrimination technique to an impulse radio system with 3 co-located interferers of equal transmit power as the desired user. The average BER performance remains constant for the region $T_{i n} \geq-56 \mathrm{~dB}$, where no uncorrupted chips are discarded and the system diversity gain is at its maximum. The perfect power control 
system keeps the co-located interferer and desired power levels equal such that interferer collisions do not overwhelm the bit decision metric $Z_{b i t}$ to produce errors at the detector. As $(I / S)_{T X}$ increases we consider the environment to have an increasingly large near-far power ratio in which the chip-discrimination technique flourishes.

The distance between the peak average BER performance and no chip discrimination at $T_{\text {in }}>-12 \mathrm{~dB}$ can be considered a performance metric for the chip-discrimination technique; it increases three orders of magnitude over the range of $(I / S)_{T X}$ from 0 to $50 \mathrm{~dB}$. We can also observe the effects of the co-located interferer transmitter power level on system performance by holding the threshold $T_{\text {in }}$ constant and viewing the specific average BER performance for different $(I / S)_{T X}$ levels. Fig. 6.4 illustrates a single slice of Fig. 6.3 along the threshold axis at $T_{\text {in }}=-56 \mathrm{~dB}$ or the threshold that achieves the maximum BER performance when $(I / S)_{T X}=40 \mathrm{~dB} . T_{\text {in }}=-56 \mathrm{~dB}$ is within the optimal BER threshold region

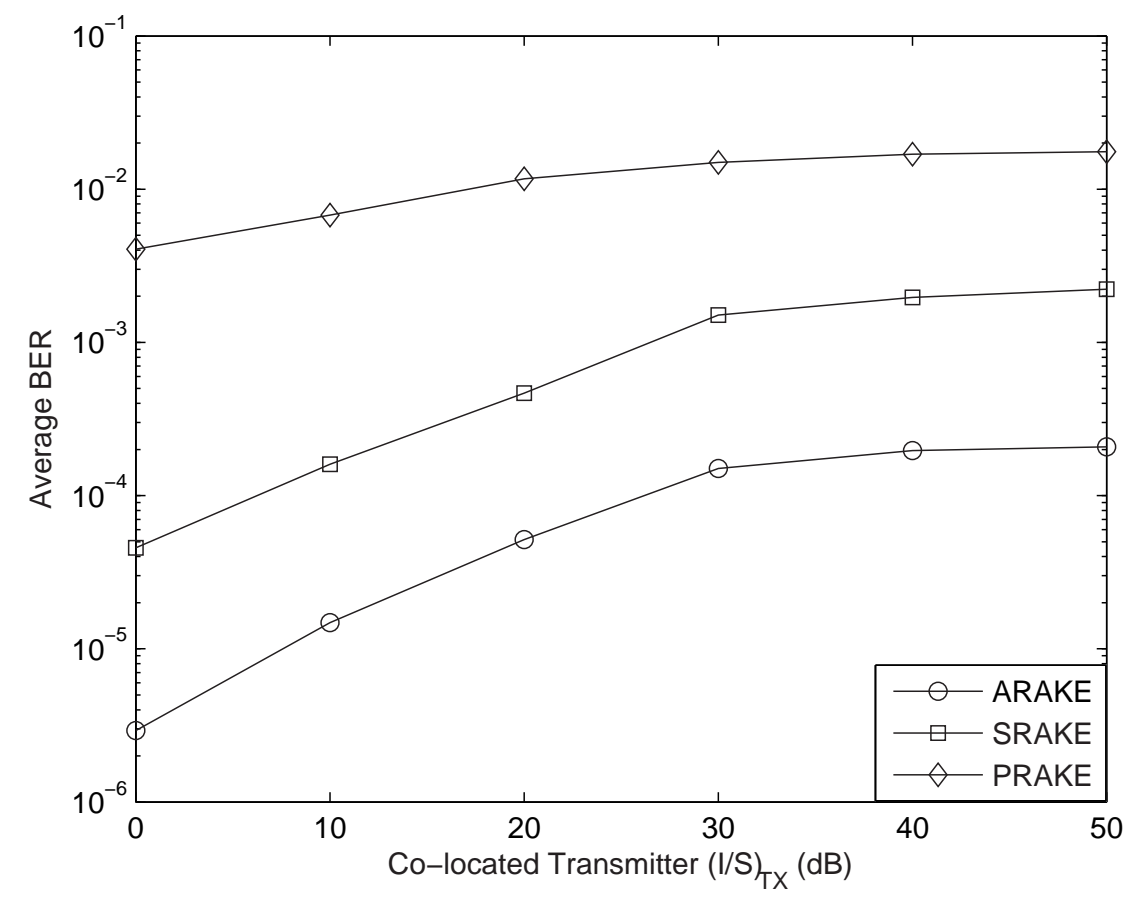

Figure 6.4: BER performance at a constant threshold $T_{i n}=-56 \mathrm{~dB}$ for varying $(I / S)_{T X}$ from $0 \mathrm{~dB}$ to $50 \mathrm{~dB}$.

for all interferer power levels investigated except the equal power case $(I / S)_{T X}=0 \mathrm{~dB}$. 
When perfect power control is achieved, a threshold $T_{\text {in }}$ of $-56 \mathrm{~dB}$ does not effectively separate the uncorrupted and corrupted chips; the increasing interferer power levels $(I / S)_{T X}$ allow the interferer chips to be more reliably discarded as described in Section 6.3. The region of interest is when $(I / S)_{T X}$ achieves the exceedingly large $(I / S)_{T X}$ range from 30 to $50 \mathrm{~dB}$. The average BER performance remains nearly constant for each RAKE receiver variety investigated in Fig. 6.4 when huge power disparities exist. In environments of smaller power disparity $\left((I / S)_{T X} \leq 20 \mathrm{~dB}\right)$, the chip discrimination performance gain is not as pronounced. In the large near-far ratio environments where chip discrimination thrives, the system exhibits little sensitivity to $(I / S)_{T X}$ as long as $T_{i n}$ is within the optimal BER region defined in Section 6.1.

We previously described a tradeoff between increased average BER performance and decreased throughput of an impulse radio system with respect to Fig. 6.2. As described in Section 2.2, when large near-far power disparity exists, a corrupted finger output $Z_{k}$ can continue to corrupt $Z_{b i t}$ regardless of the spreading sequence $N_{s}$. We use simulation to demonstrate the system average BER sensitivity to a varying number of $N_{s}$ transmitted chips per bit. We simulate the system BER performance at its optimal BER threshold $T_{\text {in }}$ using the parameters listed in Table 4.1 where $N_{s}$ varies from 1 to 100 . The results are illustrated in Figure 6.5 for the A-RAKE, S-RAKE, and P-RAKE varieties.

The average background SNR, $\overline{S N R_{b g}}$, is calibrated for each value of $N_{S}$ such that it is held constant at $34 \mathrm{~dB}$. The average energy per bit $\overline{E_{b}}$ at the RAKE combiner output increases with $N_{s}$ according to Equation 4.1. Fig. 6.5 illustrates that the three RAKE varieties investigated experience considerable performance gain as $N_{s}$ ranges from 1 to 20 chips per bit; over this range the average BER decreases approximately three orders of magnitude from 0.2 to $4 \times 10^{-4}$ for the A-RAKE receiver. We can directly compare the average BER at $N_{s}=20$ from Fig. 6.2 where no chip discrimination is employed to Fig. 6.5 to see an increase of more than three orders of magnitude from 0.4 to $4 \times 10^{-4}$. When $N_{s}$ increases beyond 20 towards 100 chips per bit, improved average BER performance is achieved at a much slower rate. The increased performance achieved for the P-RAKE is minimal for $N_{s}$ 


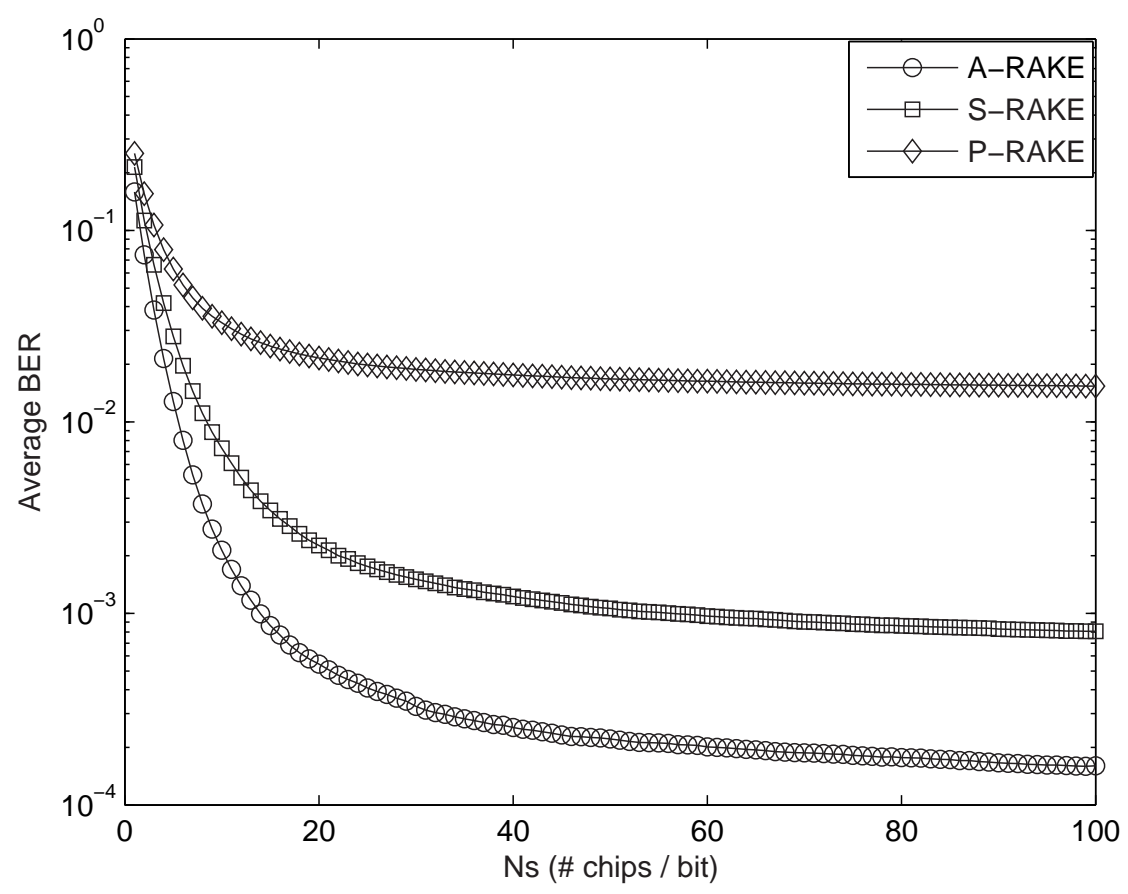

Figure 6.5: Average BER versus $N_{s}$ from 1 to 100 chips/bit at the optimal BER threshold $T_{i n}$.

larger than 30 since it becomes nearly constant. Both the S-RAKE and A-RAKE varieties continue to experience enhanced average BER performance as $N_{S}$ increases beyond $30 \mathrm{~dB}$, but results are insignificant compared to the decrease in throughput according to Equation 2.3. In fact, at the optimal BER thresholds $T_{i n}$, increasing $N_{s}$ from 50 to 100 improves performance by less than $1 \times 10^{-4}$ for the A-RAKE and $1 \times 10^{-3}$ for the S-RAKE. The minute improvements in average BER performance come with the cost of reducing system throughput $R_{s}$ by half from 2 Mbps to 1 Mbps.

The previous results vary the interference-to-signal ratio $(I / S)_{T X}$ and the spreading sequence $N_{s}$ while holding the multi-user density constant. In the military ad hoc networks of interest, nodes may enter and leave the network arbitrarily; therefore, it is necessary to quantify the effects of the co-located interferer density on the performance of an impulse radio system employing chip discrimination. Fig. 6.6 illustrates the average BER achieved by the optimal $T_{i n}$ for a varying number of co-located interferers $N_{N e a r} ;(I / S)_{T X}$ remains 
constant at $40 \mathrm{~dB}$ as well as $N_{s}$ at 100 chips per bit. In the case of $N_{N e a r}=0$, only back-

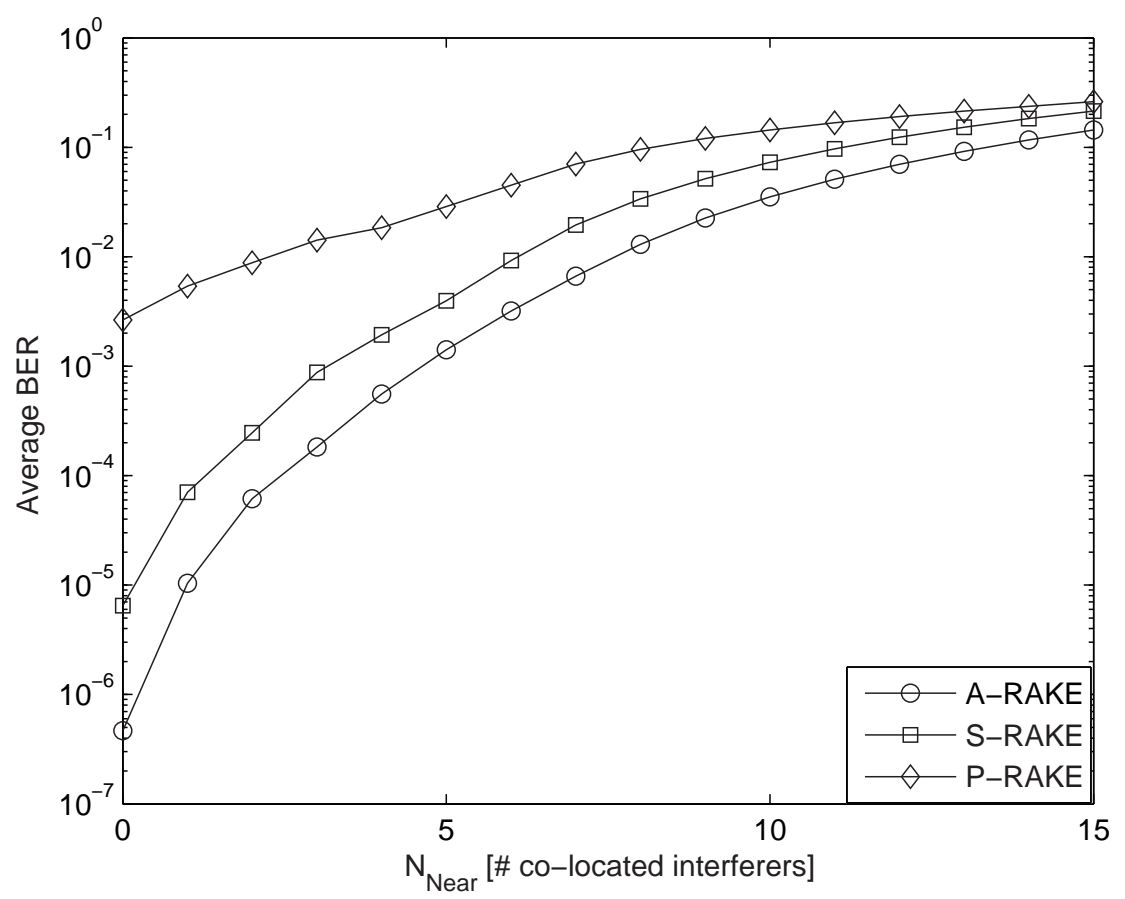

Figure 6.6: Average BER versus $N_{\text {Near }}$ from 0 to 15 co-located interferers with $(I / S)_{T X}=40 \mathrm{~dB}$.

ground noise degrades BER performance which defines the BER floor at approximately $5 \times 10^{-7}$.

When the co-located interferer density $N_{\text {Near }}$ is increased, system performance quickly degrades from the BER floor to beyond 0.1 at 15 strong interferers. When $N_{N e a r}$ increases beyond 5 users, the chip-discrimination technique can no longer achieve the target BER $10^{-3}$; however, even when 15 interfering nodes are active, the optimal $T_{i n}$ system performance still exceeds that of the equivalent system with no chip discrimination as seen Fig. 6.2. If we slice Fig. 6.6 along the x-axis at $N_{\text {Near }}=3$ interferers, the values correspond to the optimal average BER of Fig. 6.1 at $T_{i n}=-56 \mathrm{~dB}$. 


\subsection{Reliability of Discarding Chips}

In Section 6.1 we describe the chip-discrimination technique on the basis of increased BER performance at different threshold levels $T_{i n}$ without considering the reliability with which the RAKE finger outputs $Z_{k}$ are discarded. Instead of analyzing only the BER performance of a given system configuration at various thresholds $T_{i n}$, we reinforce the results of Section 6.1 by examining the probability of discarding a received chip $\left(P_{d i s}\right)$ at different $T_{\text {in }}$ values. We extend the examination in Section 6.2 to investigate the system discarding sensitivity to varying multi-user densities $N_{N e a r}$ and interferer transmit power levels $(I / S)_{T X}$; only multi-user co-located interference is considered. When necessary we analyze the probability of discarding chips for the three RAKE varieties: A-RAKE, S-RAKE, and P-RAKE. Otherwise we attempt to keep the plots as concise as possible by including only the A-RAKE results. This section presents no new information; results complement Sections 6.1 and 6.2 by reinforcing previous conclusions from a different viewpoint than average BER.

We consider the detrimental effects of discarding received chips; the BER performance increases by removing abnormally large finger output metrics $Z_{k}$ from the bit estimate metric, but the output SNR is decreased because the received bit energy is reduced. The probability of discarding a chip has a delicate relationship with $T_{i n}$ that can most easily be observed in the ideal cases of no co-located interference shown in the leftmost plots of Fig. 6.7. The simulation results for the A-RAKE, S-RAKE, and P-RAKE varieties with no multi-user interference or noise contributions illustrate the probability of incorrectly discarding a received MPC that exceeds the threshold calculated in Equation 5.6. We observe that when $T_{i n}$ is greater than approximately $-56 \mathrm{~dB}$, no chips are incorrectly discarded; however, when $T_{\text {in }}$ decreases beyond approximately $-56 \mathrm{~dB}$, MPCs are incorrectly discarded with increasing probability.

It is no coincidence that the optimal average BER region described in Section 6.1 straddles $T_{\text {in }}=-56 \mathrm{~dB}$ where $P_{\text {dis }}=0$ for $T_{\text {in }}>-56 \mathrm{~dB}$ and $P_{d i s}$ becomes increasingly nonzero for $T_{i n}<-56 \mathrm{~dB}$ as seen in Fig. 6.7. If we compare the average BER performance in 


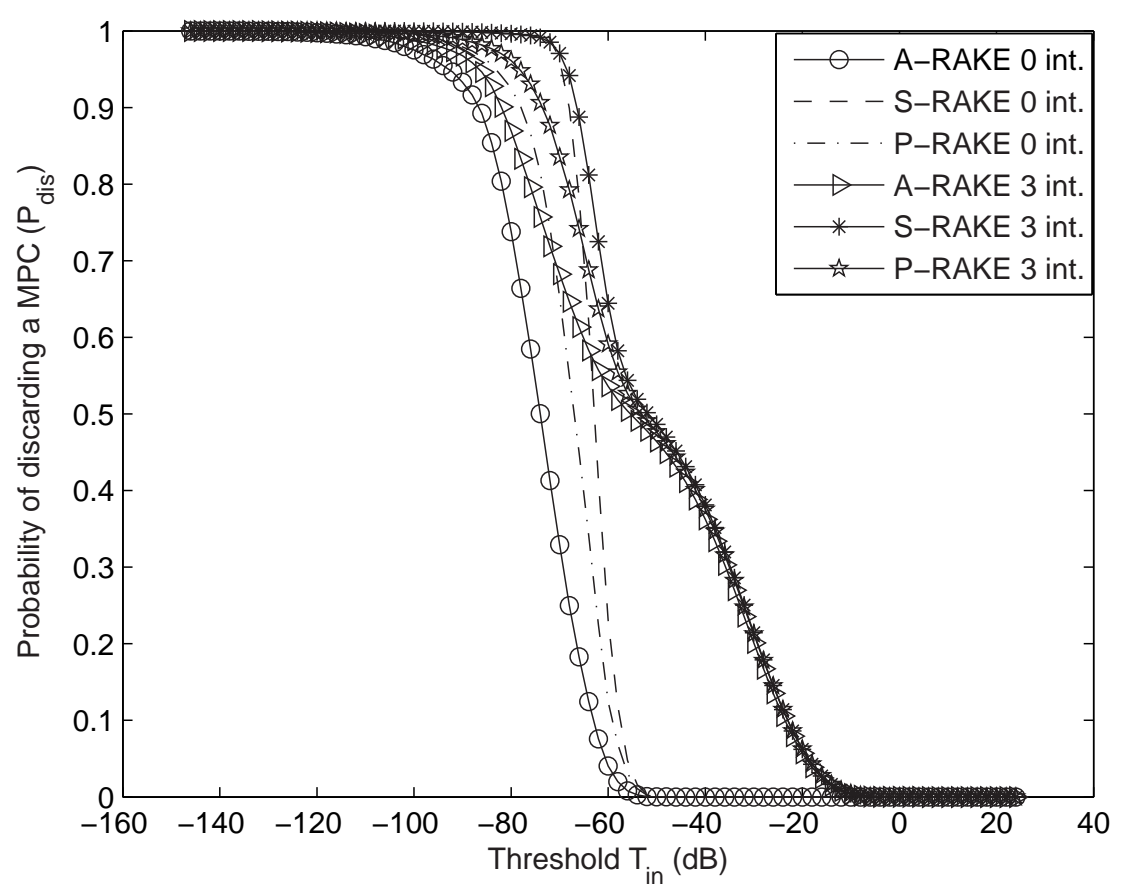

Figure 6.7: The probability of discarding chips $\left(P_{d i s}\right)$ with the A-RAKE, S-RAKE, and P-RAKE receivers for varying threshold levels $T_{i n}$ applied to the RAKE finger output $Z_{k}$. The curves correspond to co-located multi-user densities $N_{\text {Near }}=0$ and 3 interferers with $(I / S)_{T X}=40 \mathrm{~dB}$.

Fig. 6.1 with the probability of incorrectly discarding chips in Fig. 6.7, we see that as $T_{\text {in }}$ decreases from approximately $-12 \mathrm{~dB}$, the BER continuously decreases until its peak performance value at $T_{i n}=-56 \mathrm{~dB}$. In the range of $T_{i n} \geq-56 \mathrm{~dB}$, no chips are incorrectly discarded because $T_{i n}$ is large enough to allow all valid resolved MPCs to pass through the chip discriminator unaltered; only an interferer collision would increase $\left|Z_{k}\right|$ enough to be discarded. As $T_{\text {in }}$ decreases beyond $-56 \mathrm{~dB}$, chips are more frequently discarded incorrectly until $T_{\text {in }}=-120 \mathrm{~dB}$, where all chips are discarded for the three RAKE receivers investigated. The region $T_{i n} \leq-120 \mathrm{~dB}$ forces the chip discriminator to discard all $Z_{k}$ and the detector makes an equiprobable bit estimate resulting in 0.5 average BER.

We have thus far limited the discussion in this section to comparing the probability of discarding chips to the simulation results in Section 6.1 where $N_{N e a r}=3$ and $(I / S)_{T X}=$ $40 \mathrm{~dB}$. We now take an approach much like Section 6.2 to investigate the effects of the 
simulation parameters $N_{\text {Near }}$ and $(I / S)_{T X}$ on the probability of discarding a chip. We only illustrate the A-RAKE receiver results since all investigated receiver curves follow the same trends seen in Fig. 6.7 where the three RAKE probability curves coincide when $T_{\text {in }}>-56 \mathrm{~dB}$ and differ for $T_{\text {in }}<-56 \mathrm{~dB}$. We begin by analyzing the probability of discarding a chip $\left(P_{d i s}\right)$ for varying $T_{i n}$, where $N_{\text {Near }}$ co-located interferers exist with interferenceto-signal ratio $(I / S)_{T X}=40 \mathrm{~dB}$. Fig. 6.8 gives the probability curves for $N_{N e a r}=0,3,7$, and 10 co-located interferers.

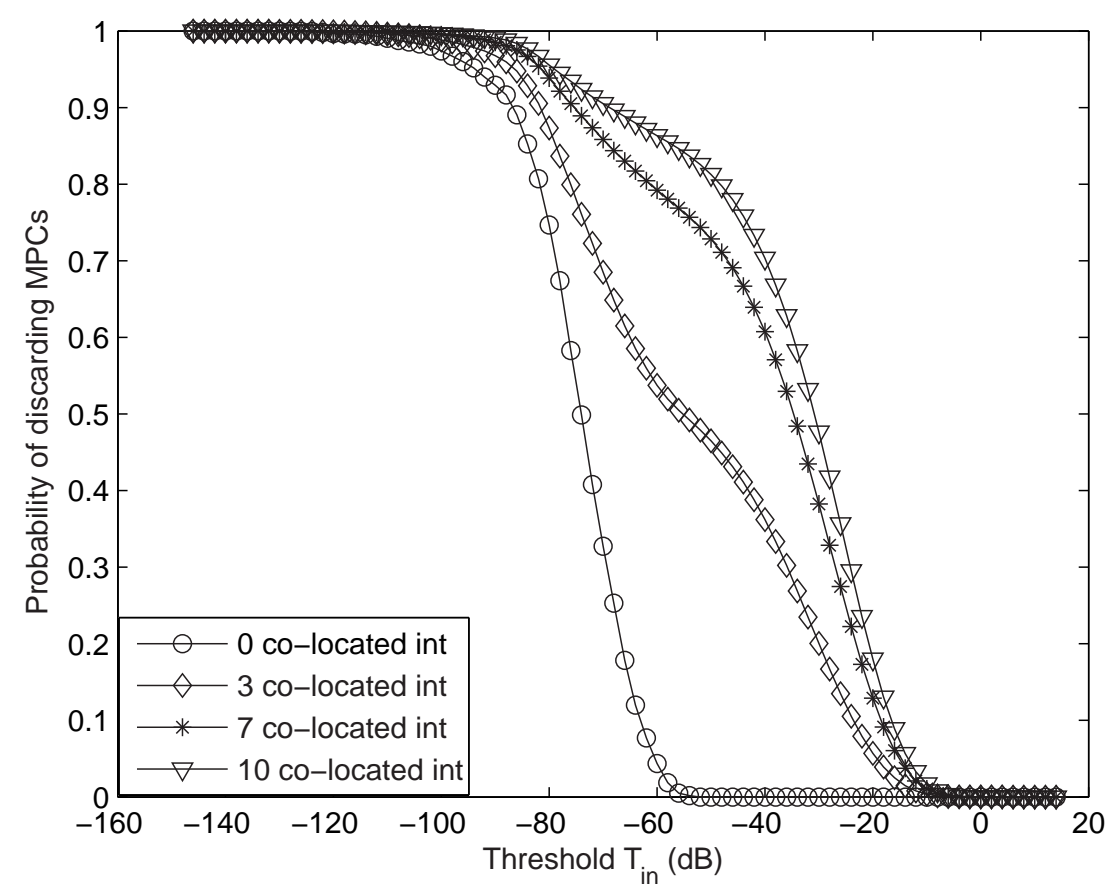

Figure 6.8: The probability of discarding chips $\left(P_{d i s}\right)$ with the A-RAKE receiver for varying threshold levels $T_{i n}$ applied at the RAKE finger output. The co-located interferer multi-user density $N_{\text {Near }}$ ranges from 0 to 10 interferers with $(I / S)_{T X}=40 \mathrm{~dB}$.

When the interferer transmitter power is excessively large as considered for $(I / S)_{T X}=$ $40 \mathrm{~dB}$, increasing $N_{N e a r}$ extends the region of nonzero probability of discarding a chip to increasingly large values of $T_{i n}$. This is to be expected following Equation 5.5 where the probability of avoiding a collision decreases exponentially according to $N_{N e a r}$. As the number of co-located interferers $N_{N e a r}$ increases, more chips are corrupted by collisions 
and a threshold $T_{i n}$ larger than $-56 \mathrm{~dB}$ can more effectively discard them. In the ideal case, the set of discarded chips remains empty until $T_{i n}$ decreases below $-56 \mathrm{~dB}$ and chips are steadily added to the set of discards. The larger values of $N_{N e a r}$ allow corrupted chips to be discarded with larger values of $T_{\text {in }}$ which leaves fewer chips to be discarded after $T_{\text {in }}$ decreases beyond $-56 \mathrm{~dB}$. Hence as $N_{\text {Near }}$ increases, the nonzero probability region extends to larger values of $T_{i n}$ and $P_{d i s}$ achieves unity for larger $T_{i n}$. The slope of the probability curve becomes steeper for increasing $N_{\text {Near }}$ because fewer small values of $T_{\text {in }}$ are necessary to discard the increasingly corrupt MPCs.

The large received power level of the multi-user interferers in Fig. 6.8 compared to the desired received chip power level when $(I / S)_{T X}=40 \mathrm{~dB}$ allows the RAKE finger outputs $Z_{k}$ corrupted by interferer collisions to be more reliably discarded at larger $T_{\text {in }}$ from the bit estimate metric $Z_{b i t}$. To better understand the role of $(I / S)_{T X}$ in determining the probability of discarding a chip, we examined the sensitivity of $P_{d i s}$ to a varying range of co-located interferer power levels. Fig. 6.9 illustrates simulation results for $P_{d i s}$ in a system with three co-located interferers and a range of $(I / S)_{T X}$ from 0 to $40 \mathrm{~dB}$.

The probability of discarding a chip $\left(P_{d i s}\right)$ at different levels of $(I / S)_{T X}$ closely follows the behavior of Fig. 6.8 for increasing co-located interference levels $N_{N e a r}$. The region of nonzero $P_{d i s}$ extends to larger values of $T_{i n}$ when $(I / S)_{T X}$ increases. Fig. 6.9 demonstrates the efficacy of the chip-discrimination technique in environments with increasing near-far power ratio. Chips are discarded with more certainty in large interference-to-signal ratio environments because the thresholds applied have less chance of erroneously discarding an uncorrupted chip. As $(I / S)_{T X}$ increases, corrupted $\left|Z_{k}\right|$ grow, but decreasing $T_{\text {in }}$ beyond $-62 \mathrm{~dB}$ has little effect on $P_{d i s}$; all $Z_{k}$ resulting from large interferer collisions have already been discarded at larger $T_{\text {in }}$ such that the curves nearly coincide as the probability of discarding a chip approaches unity. This comes as no surprise after reviewing Fig. 6.3 where the BER performance curves are nearly equivalent for all $(I / S)_{T X}$ when $T_{\text {in }}<-62 \mathrm{~dB}$.

In the ideal case of no multi-user interference, the probability of discarding a chip from Fig. 6.7 with the A-RAKE receiver matches closely to the perfect power control scenario of 


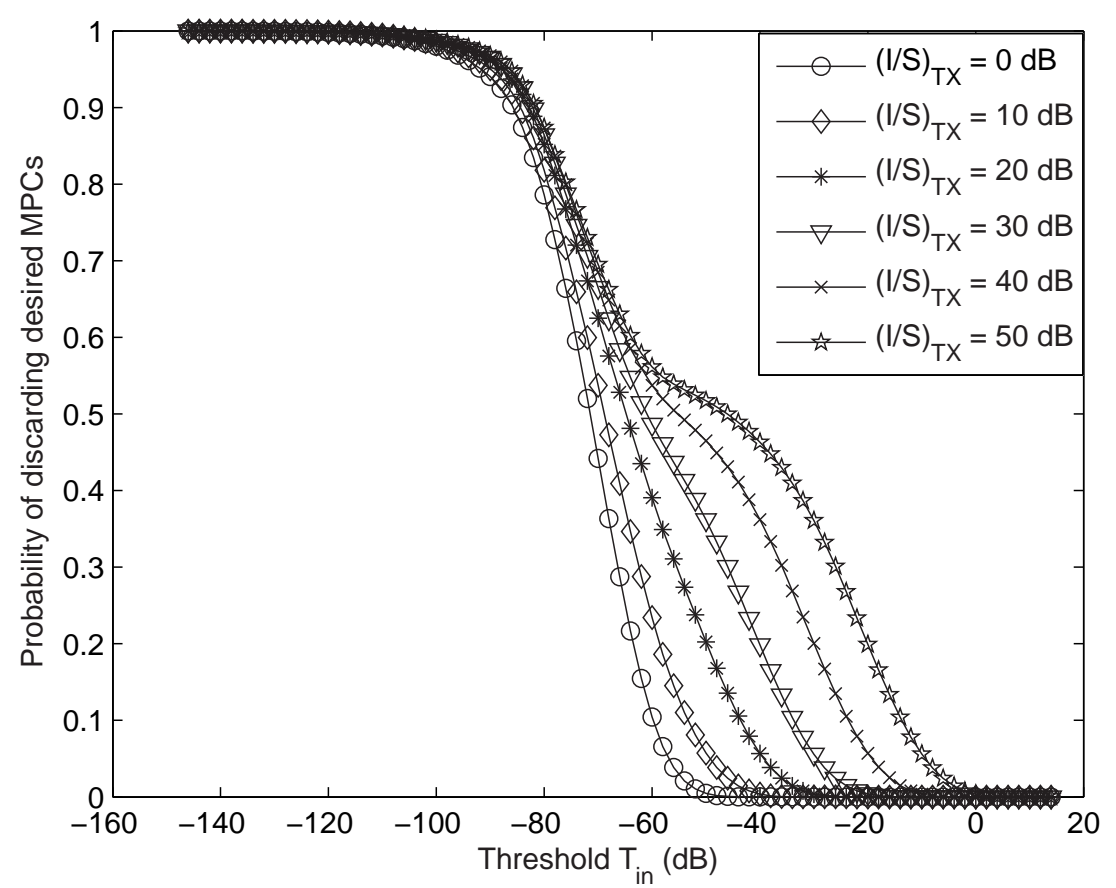

Figure 6.9: The probability of discarding chips $\left(P_{\text {dis }}\right)$ with the A-RAKE receiver for varying threshold levels $T_{\text {in }}$ applied at the RAKE finger output. The co-located interferer multi-user density $N_{\text {Near }}$ is constant at 3 interferers with $(I / S)_{T X}$ ranging from $0 \mathrm{~dB}$ to $40 \mathrm{~dB}$.

three co-located interferers where $(I / S)_{T X}=0 \mathrm{~dB}$ in Fig. 6.9. The channel diversity gain achieved with the RAKE receiver varieties allows the system with perfect power control to recover from bit errors without chip discrimination. This reinforces the conclusion first presented in [1] and reiterated throughout this work that the increased system complexity in implementing the chip discrimination technique is only beneficial in large near-far power ratio environments.

\subsection{Imperfect Channel Estimation}

The previous results of this chapter assume perfect channel estimation of tap delays $\tau_{k}$ and coefficients $\alpha_{k}$. In general this is not possible because of constant fluctuations in the multipath environment that cause different levels of inaccuracy in channel estimates. For the impulse radio system presented in this thesis, the accuracy of channel tap coefficient 
estimates affects performance in combining the individually resolved multipath component (MPC) metrics $Z_{k}$ using maximal ratio combining (MRC) according to Equation 3.19. The chip discriminator that operates on each RAKE receiver finger output $Z_{k}$ following Equation 5.6 also requires channel coefficient estimates. Perfect estimation of the channel tap coefficients $\alpha_{k}$ is required by both the RAKE combiner and the chip discriminator to achieve the optimal average BER performance. We apply a method presented in [7] to the impulse radio system model employing chip discrimination to investigate the effects of imperfect channel tap coefficient estimation on system BER performance. The assumption of perfect estimation of channel tap delays $\tau_{k}$ still holds throughout this section.

We model imperfect channel tap coefficients by corrupting the perfect coefficients $\alpha_{k}$ with the addition of an independent Gaussian estimation error term $E_{\text {err }}$, where $E_{\text {err }}$ has zero-mean and variance $\sigma_{e r r}^{2}$ specified according to

$$
S N R_{e r r}=\frac{\max \left(\left(\alpha_{k}^{i}\right)^{2}\right)}{\sigma_{e r r}^{2}}
$$

The estimation error variance $\sigma_{e r r}^{2}$ is chosen based on the $L$ perfect channel estimates of a given channel realization. The resulting imperfect coefficient estimate for the $k^{\text {th }}$ coefficient of the $i^{t h}$ channel realization is given by $\tilde{\alpha_{k}^{i}}=\alpha_{k}^{i}+E_{\text {err }}^{(k)}$ [7]. The imperfect coefficients $\tilde{\alpha_{k}}$ are computed from the perfect channel estimates for each RAKE receiver variety. We use computer simulation to analyze the average BER performance for the impulse radio system specified by the parameters of Table 4.1 when channel tap coefficients are estimated incorrectly and chip discrimination is employed. We compare performance results for varying levels of $S N R_{e r r}$ to the benchmark results of Section 6.1 where perfect coefficient estimates are available. The results for each RAKE receiver variety are plotted separately to make the graphs readable; however, each receiver exhibits the same fundamental behavior such that discussions can be considered applicable to all investigated RAKE receivers.

We present the average BER performance results for the A-RAKE receiver in Figure 6.10, followed by the S-RAKE and P-RAKE results in Figures 6.11 and 6.12 respec- 
tively. The average BER performance at $S N R_{\text {err }}=40 \mathrm{~dB}$ nearly coincides with the op-

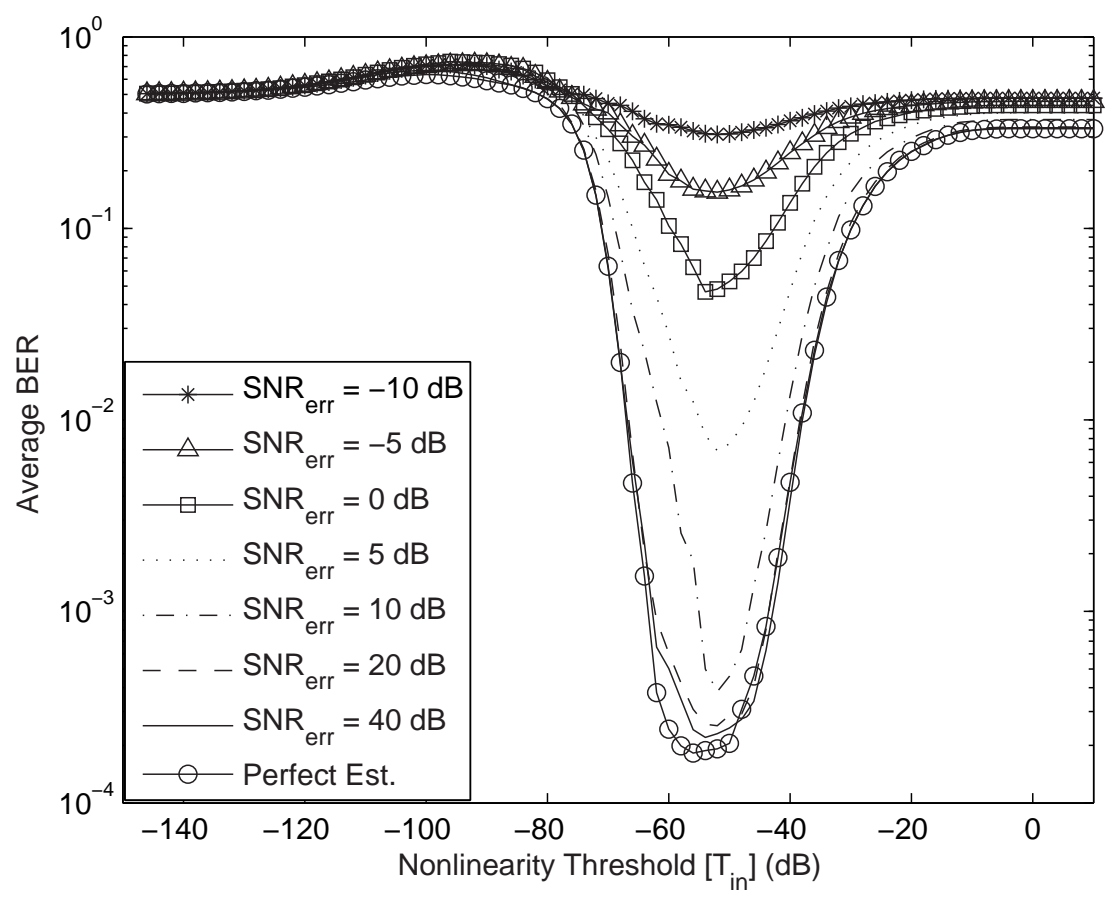

Figure 6.10: Average BER achieved for a chip discriminator threshold $T_{i n}$ varying between $-126 \mathrm{~dB}$ and $-6 \mathrm{~dB}$ and imperfect A-RAKE channel coefficient estimation specified by $S N R_{e r r}$.

timal performance curve for perfectly estimated channel tap coefficients for all RAKE varieties. In fact, $S N R_{e r r}=20 \mathrm{~dB}$ also results in an average BER very close to that of the perfect estimation case. This is to be expected since the noise variance $\sigma_{e r r}^{2}$ is small for these large values of $S N R_{\text {err }}$; however, as $S N R_{\text {err }}$ decreases beyond $10 \mathrm{~dB}$, the performance of the A-RAKE receiver decays quickly until chip discrimination provides no increase in BER performance. The S-RAKE and P-RAKE systems are more resilient to decreasing $S N R_{\text {err }}$ than the A-RAKE receiver; only slight losses in BER performance are incurred for $S N R_{\text {err }}=40 \mathrm{~dB}, 20 \mathrm{~dB}$, and $10 \mathrm{~dB}$. When $S N R_{\text {err }}$ decreases beyond $5 \mathrm{~dB}$, the average BER performance decays quickly as in the A-RAKE case. For all RAKE varieties analyzed, decreasing $S N R_{\text {err }}$ beyond approximately $-15 \mathrm{~dB}$ reduces BER performance of a system with chip discrimination to that of an equivalent system without.

Incorrectly estimated channel coefficients affect BER performance in impulse radio 


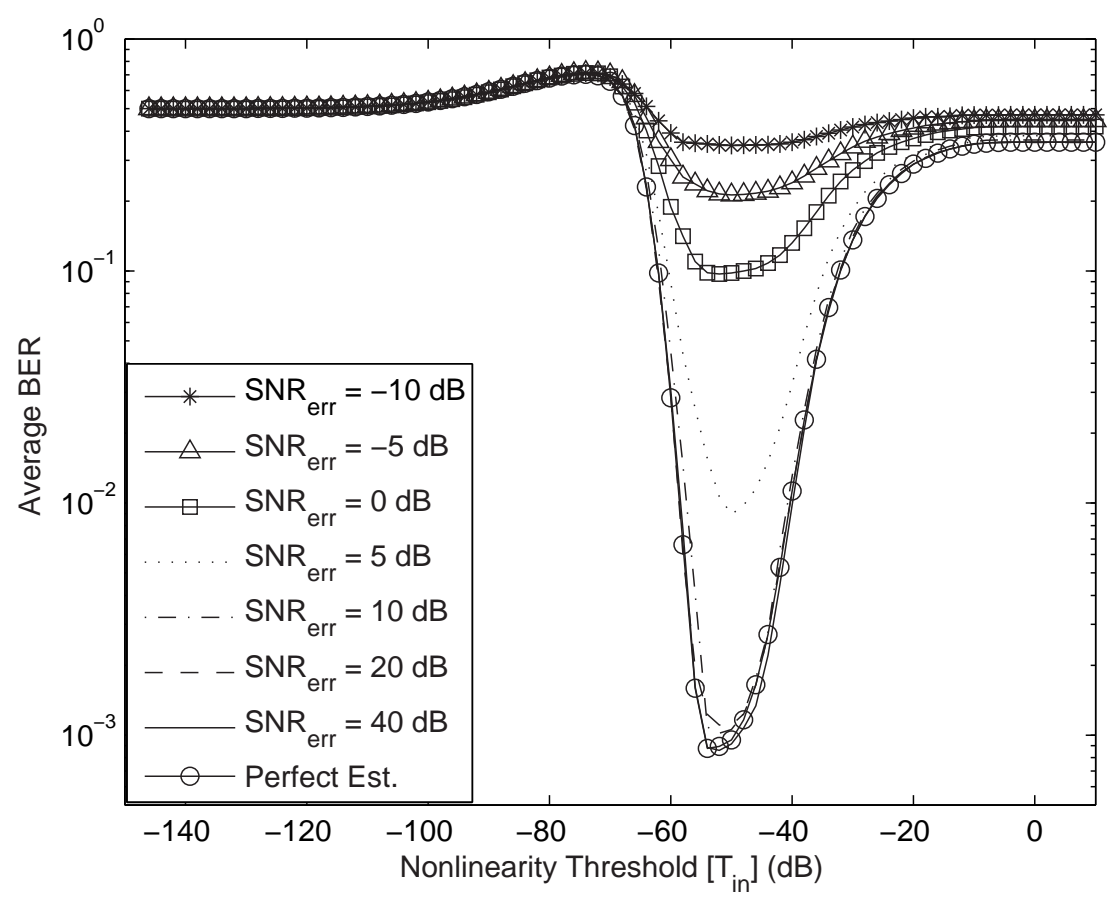

Figure 6.11: Average BER achieved for a chip discriminator threshold $T_{\text {in }}$ varying between $-126 \mathrm{~dB}$ and $-6 \mathrm{~dB}$ and imperfect S-RAKE channel coefficient estimation specified by $S N R_{e r r}$.

systems that employ chip discrimination as well as those that do not. Each individually resolved MPC is weighted by its corresponding tap coefficient $\alpha_{k}$ using MRC. For chip discriminator threshold levels $T_{\text {in }} \geq-12 \mathrm{~dB}$, no chip discrimination is applied to the RAKE finger outputs, yet the average BER exceeds that of the perfectly estimated coefficient system for all RAKE varieties examined. As described in Section 6.1, the average BER with perfectly estimated channel coefficients is between 0.3 and 0.4 depending on the RAKE receiver variety. When no chip discriminator is applied to the A-RAKE finger outputs $Z_{k}$, an average BER of approximately 0.33 is achieved; the S-RAKE and P-RAKE achieve approximately 0.358 and 0.392 respectively. The values of $S N R_{\text {err }}$ greater than $10 \mathrm{~dB}$ achieve nearly the average BER of the perfectly estimated coefficients in the A-RAKE system with no chip discrimination. As $S N R_{\text {err }}$ decreases beyond $5 \mathrm{~dB}$, the average BER decays to 0.5 . As $\sigma_{e r r}^{2}$ increases, the sign of $\tilde{\alpha_{k}}$ can differ from $\alpha_{k}$ with increased frequency, pushing the average BER to 0.5. The S-RAKE and P-RAKE performance follows that of the A-RAKE, but average BER does not decay to 0.5 until $S N R_{\text {err }}$ decreases below $0 \mathrm{~dB}$. 


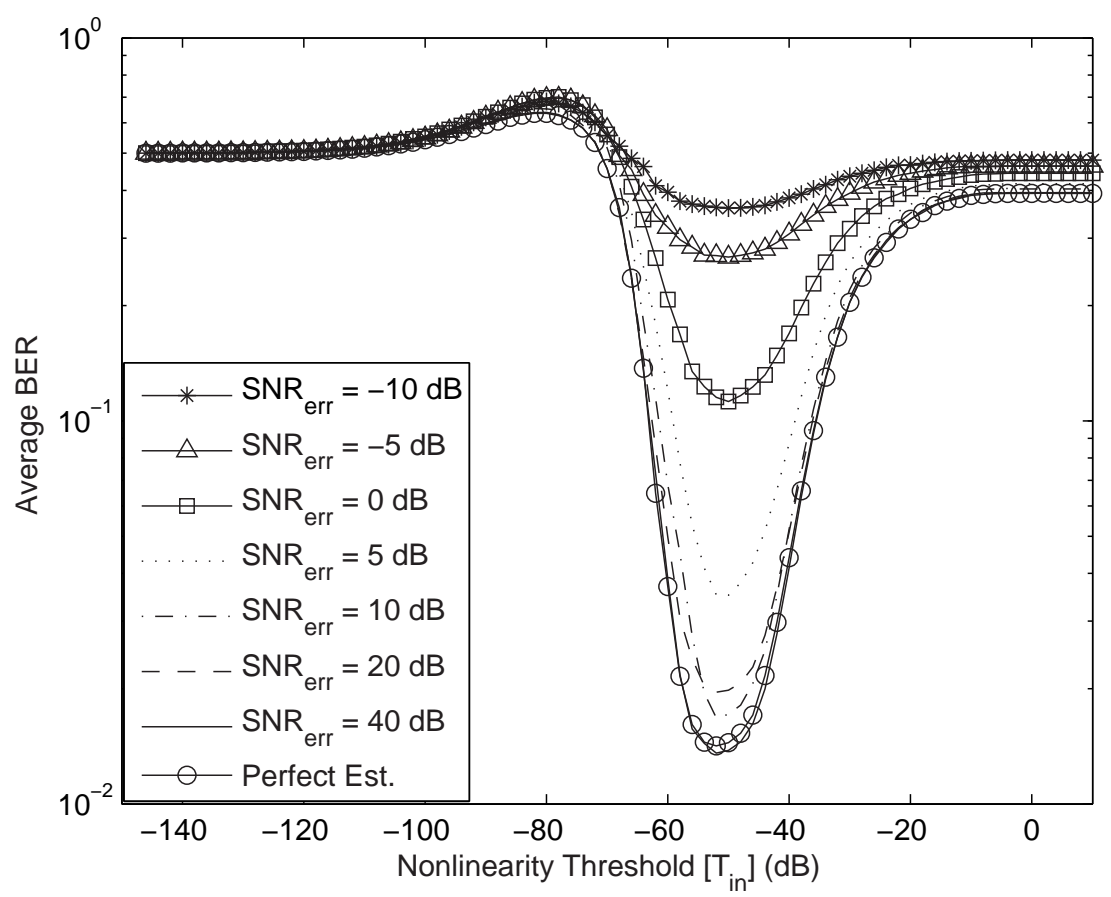

Figure 6.12: Average BER achieved for a chip discriminator threshold $T_{\text {in }}$ varying between $-126 \mathrm{~dB}$ and $-6 \mathrm{~dB}$ and imperfect P-RAKE channel coefficient estimation specified by $S N R_{e r r}$.

The elevated BER region as described in Section 6.1 occurs when $T_{\text {in }}$ is between $-70 \mathrm{~dB}$ and $-120 \mathrm{~dB}$ and a lack of uncorrupted demodulation metrics $Z_{k}$ drives the average BER above 0.5 . In the case of the A-RAKE receiver, the incorrectly estimated channel coefficients $\tilde{\alpha_{k}}$ magnify the detrimental effects experienced in the elevated BER region shown in Fig. 6.10. The surviving $\left|Z_{k}\right|$ of the original $L$ resolved with the A-RAKE receiver must be extremely small to avoid being discarded for the small threshold values $T_{\text {in }}$ considered. The resulting bit metric $Z_{b i t}$ is computed from only a minimal set of surviving $Z_{k}$ collected over $N_{s}$ chips that possibly consists of all corrupted metrics. The small energy paths collected by the A-RAKE allow $E_{\text {err }}$ to change the sign of the perfectly estimated coefficients $\alpha_{k}$ such that scaling the surviving $Z_{k}$ by $\tilde{\alpha_{k}}$ increases the frequency of corrupted bit metrics $Z_{b i t}$. Hence the average BER calculated from a majority of incorrect $Z_{b i t}$ is forced above 0.5 for the binary impulse radio system analyzed. 
The P-RAKE receiver experiences only a slight elevation in average BER due to imperfect channel estimates shown in Fig. 6.12 when $T_{\text {in }}$ is between $-70 \mathrm{~dB}$ and $-95 \mathrm{~dB}$. The largest channel coefficients generally arrive first due to the exponentially decaying power delay profile of the channel allowing the majority of $Z_{k}$ to be discarded within the elevated BER region. The incorrectly estimated coefficients $\tilde{\alpha_{k}}$ bias the surviving $Z_{k}$, but the average BER suffers only a small increase. The sign of $\tilde{\alpha_{k}}$ does not frequently differ from the perfectly estimated coefficients $\alpha_{k}$ for the P-RAKE because paths of small energy are generally not resolved. The corrupted channel coefficients $\tilde{\alpha_{k}}$ slightly increase the average BER already elevated by multi-user interference and a dearth of uncorrupted $Z_{k}$. Following the same rationale, the S-RAKE suffers no decreased BER performance in the elevated BER region shown in Fig. 6.11. The MPCs corresponding to the largest $L_{s}$ paths generally produce $Z_{k}$ that surpass the chip discriminator threshold and become discarded. Only a collision with interfering chips can decrease $\left|Z_{k}\right|$ enough to survive the chip discriminator. The incorrectly estimated coefficients $\tilde{\alpha}_{k}$ scale the surviving $Z_{k}$ without altering its sign and hence the average BER remains constant for the perfectly and imperfectly estimated channel coefficients considered. 


\section{Chapter 7}

\section{Conclusions and Future Work}

The ultra-wideband impulse radio physical layer provides a naturally covert time-hopping solution for short-range, multi-user military ad hoc networks, but requires accurate power control algorithms. Previous power control methods have been investigated specific to impulse radio systems to mitigate near-far power disparities, but they require coordination from network users which increases system complexity and latency. These solutions may fail in the case of network nodes belonging to hostile users and malicious jamming. An autonomous power control solution that requires no coordination from other network nodes is needed to overcome detrimental effects of multi-user interference with few increases in system complexity and latency.

An autonomous chip-discrimination technique was presented in [1] that considered the increased system performance when applying an acceptance threshold to the matched filter output of the Gaussian impulse radio channel. The results demonstrated that the chipdiscrimination technique thrives in large near-far power ratios where target BERs of $10^{-3}$ can be achieved for multi-user densities of 10 high-powered co-located users. In this thesis we extended the work of [1] by applying the basic chip-discrimination technique to the IEEE 802.15.3a UWB indoor multipath channel model. We first presented the basic background information and terminology concerning an impulse radio physical layer system. A 
summary of the autonomous chip-discrimination technique is given specific to the binary pulse position modulation (BPPM) system considered throughout this work.

A considerable amount of effort was given to establish the recently adopted IEEE 802.15.3a indoor channel model as it applies to the simulation model presented in this thesis. We began with the $\mathrm{S}-\mathrm{V}$ wideband model and used the basic definitions to develop the IEEE 802.15.3a channel model. Information is easily obtained in literature concerning the UWB channel model, but few sources describe converting the continuous-time IEEE 802.15.3a channel model to a discrete-time impulse response that can be used in simulation. We presented the fundamental steps that yield the IEEE 802.15.3a discrete-time impulse response with taps spaced every integer multiple of the inverse pulse bandwidth $T_{p}$.

Although robust, there are inherent limitations that restrict the situations for which UWB system analysis with the channel model is valid. Specifically, in the two models presented in this work, CM1 and CM2, the maximum Tx-Rx separation is $4 \mathrm{~m}$ and no spatial correlation is incorporated into the model. The IEEE 802.15.3a model parameters are derived by matching model simulation parameters with measured data results; we use simulation to quantify the simulated IEEE 802.15.3a multipath channel model in terms of the standard statistical multipath parameters. A description of the maximal ratio combining RAKE receiver is given as well as the three varieties of RAKE receivers that are analyzed in this thesis: A-RAKE, S-RAKE, and P-RAKE.

A simulation model that captures the essence of the investigated network and satisfies the channel model restrictions was necessary to test the chip-discrimination technique in a multi-user multipath environment; however, few results are available modeling a specific multi-user environment using the IEEE 802.15.3a channel model. We created a model environment containing three co-located interferers with transmit power much larger than the desired user such that a large near-far power ratio is produced. An unspecified number of background interferers with transmit power level equal to the desired user combined with thermal noise at the receiver maintain an average output SNR of $34 \mathrm{~dB}$. The desired user transmits via $\mathrm{CM} 2$ and the co-located interferers transmit via CM1 equidistant from the 
desired receiver. The novelty of the system environment required that basic assumptions be stated along with the specific system simulation parameters.

We mathematically presented the total received signal under the assumption of system linearity. This yields the sum of $L$ multipath components produced by every transmitted chip from each of the $N_{\text {near }}$ multi-user interferer signals. A RAKE receiver is used to track and demodulate the $L$ resolvable multipath components produced by the transmitted desired user. For the multipath system analyzed, we present a novel chip discriminator to be placed at the output of each RAKE receiver finger. Unusually large demodulation metrics $Z_{k}$ that exceed the defined threshold are discarded from each chip metric $Z_{\text {chip }}$.

Just as in the case of simple chip discrimination with constant threshold $X$, there exists a region of constant threshold values $T_{\text {in }}$ where system performance increases in terms of BER. Although chip discrimination can greatly improve system BER performance with minimal increases in complexity, system performance can also be shattered with a poor choice of $T_{i n}$. When $T_{\text {in }}$ is larger than approximately $-12 \mathrm{~dB}$, the system is equivalent to a system without chip discrimination where no performance gain is experienced. Furthermore, when $T_{\text {in }}$ decreases to $-100 \mathrm{~dB}$ and below, the threshold is so harsh that nearly all resolved MPC voltage metrics are discarded and BER performance decays to 0.5.

The techniques presented in this thesis could be enhanced by future investigations that remove some of the fundamental system assumptions stated in Section 4.2.1. The results presented should be considered as benchmarks for a multi-user system operating in a multipath environment. The chip discriminator and the maximal ratio combining (MRC) RAKE receiver require accurate channel estimates and performance suffers accordingly when imperfect channel parameters are obtained. Both the MRC and the Minimum Mean Squared Error (MMSE) combining techniques should be investigated; linear combining techniques such as Equal Gain Combining (EGC) provide no simplification in the chip-discrimination system presented since the channel tap coefficients $\alpha_{k}$ are needed regardless of the combining method. 
More complex channel estimation algorithms should be examined and performance compared to the Gaussian-corrupted and perfectly estimated coefficient results given in this thesis. Imperfect channel estimation should also include timing jitters in the MPC delay estimates $\tau_{k}$ such that all of the desired user's received energy is not collected in the RAKE finger correlation window. By estimating the channel with known pilot sequences, the assumption of uncorrelated RAKE fingers no longer holds true because the fingers are not independently estimated as in the case of perfect estimation.

A thorough analysis of the chip-discrimination technique presented cannot be based solely on a single channel model. We investigated CM3 and CM4 which offer increased Tx-Rx separation distance of up to $10 \mathrm{~m}$ while holding the co-located interferers constant with the LOS CM1 for all simulations. Both CM3 and CM4 model environments with many more reflections and refractions of rays than are present in CM2. The results produced curves with only slight performance gain increase over $\mathrm{CM} 2$, but interestingly the optimal BER threshold region widened as the number of resolvable desired user components increased.

Although widely adopted in current UWB literature, the IEEE 802.15.3a channel model is not the best fit for investigating the tactical military networks of interest. Future research should examine the chip-discrimination technique in an outdoor UWB channel model that more realistically simulates the wireless, military ad hoc networks of interest; however, there is presently a dearth of published outdoor UWB channel models. The IEEE 802.15.4a UWB physical layer subgroup recently published a set of preliminary outdoor channel models for simulation with UWB sensor units, but we are unaware of any published impulse radio results that use the IEEE 802.15.4a channel model.

The work presented in this thesis only considers the system data rate $R_{S}$ when necessary to compare with average BER performance. Future military ad hoc networks require data rates much larger than $R_{S}=2 \mathrm{Mbps}$ achieved for $N_{s}=50$ and $T_{f}=100 \mathrm{~ns}$ as discussed in Section 6.2. We demonstrated the performance consequences of varying $N_{s}$ with and without chip discrimination, but altering $T_{f}$ was never considered. When $T_{f}$ is decreased, ISI 
increasingly degrades average BER performance. Future investigations should examine the performance degradation of the impulse radio system employing chip discrimination due to ISI and the efficacy of equalization to mitigate the loss incurred.

In the IEEE 802.15.3a channel model examined, the chip-discrimination technique provided exceptional mitigation of multi-user interference in a network with large near-far power ratio. The diversity gain allowed the system to outperform the equivalent system in the Gaussian channel in terms of BER for a small number of co-located interferers; however as the number of co-located interferers grows, the performance quickly decays to a system where reliable communication is impossible. An impulse radio military ad hoc network that seeks to achieve a target BER for a small number of co-located interferers $(<6)$, or that seeks to mitigate the effects of a strong hostile jammer could benefit immensely from implementing the passive chip-discrimination technique. A network with larger colocated multi-user density would receive better performance from a coordinated blanking or feedback-based coordinated power control algorithm that actively adjusts transmit power levels of each node. 


\section{Bibliography}

[1] W. M. Lovelace and J. K. Townsend. Chip Discrimination for Large Near-Far Power Ratios in UWB Networks. In IEEE MILCOM 2003, Oct. 2003.

[2] D. G. Brennan. Linear Diversity Combining Techniques. In Proc. IRE, volume 47, pages 1075 - 1101, June 1959.

[3] R. A. Scholtz. Multiple Access with Time-Hopping Impulse Modulation. In Proc. IEEE MILCOM, Oct. 1993.

[4] M. Z. Win and R. A. Scholtz. Impulse radio: How it works. Communications Letters, IEEE, 2(2):36-38, 1998.

[5] Maria-Gabriella Di Benedetto, Guerino Giancola. Understanding Ultra Wide Band Radio Fundamentals. Prentice Hall PTR, Series: Prentice Hall Communications Engineering and Emerging Technologies Series, 1st edition, 2004.

[6] A. Saleh, R. Valenzuela. A Statistical Model for Indoor Multipath Propagation. IEEE Journal on Selected Areas in Communications, 5(2):128 - 137, Feb. 1987.

[7] Sumit Roy, Jeff R. Foerster, V. Srinivasa Somayazulu, Dave G. Leeper. Ultrawideband Radio Design: The Promise of High-Speed, Short-Range Wireless Connectivity. Proceedings of the IEEE, 92(2):295 - 311, Feb. 2004.

[8] J. R. Foerster, M. Pendergrass, A. F. Molisch. A Channel Model for Ultrawideband Indoor Communication. International Symposium of Wireless Personal Multimedia Communication, October 2003. 
[9] J. Foerster. Channel Modeling Subcommittee Report Final (doc.: IEEE802-15-02/490rl-SG3a). IEEE P802.15 Working Group for Wireless Personal Area Networks (WPANs), Feb. 2002. Available: http://grouper.ieee.org/groups/802/15/pub/2002/Nov02.

[10] Mohammad Azizur Rahman, Shigenobu Sasaki, Jie Zhou, Shogo Muramatsu, Hisakazu Kikuchi. On Rake Reception of Ultra Wideband Signals Over Multipath Channels From Energy Capture Perspective. IEICE Trans. Fundamentals, E88-A(9), Sept. 2005.

[11] S. S. Kolenchery, J. K. Townsend, J. A. Freebersyser, G. Bilbro. Performance of Local Power Control in Peer-to-Peer Impulse Radio Networks with Bursty Traffic. In Proc. IEEE GLOBECOM 1997, pages 910-916, Nov. 1997.

[12] I. Güvenc, H. Arslan. On the Modulation Options for UWB Systems. In Proc. IEEE MILCOM03, October 2003.

[13] Leon W. Couch. Digital and Analog Communications Systems. Prentice Hall, Inc., 6th Edition edition, 2001.

[14] T. S. Rappaport. Wireless Communications: Principles and Practice. Prentice Hall, Inc., 2nd edition, 2002.

[15] S. S. Kolenchery, J. K. Townsend, J. A. Freebersyser, G. Bilbro. A Novel Impulse Radio Network for Tactical Military Wireless Communications. Military Communications Conference, 1:59-65, Oct. 1998.

[16] Maria-Gabriella Di Benedetto Guerino Giancola, Luca De Nardis. Multi User Interference in Power Unbalanced Ultra Wide Band systems: Analysis and Verification. In IEEE Conference on Ultra Wideband Systems and Technologies, Nov. 2003.

[17] M. Z. Win and R. A. Scholtz. Ultra-Wide Bandwidth Time-Hopping Spread-Spectrum Impulse Radio for Wireless Multiple-access Communications. IEEE Trans. Commun., volume 48:679-691, April 2000. 
[18] John G. Proakis. Digital Communications. McGraw-Hill, Inc., 4th edition, 2001.

[19] Di Benedetto, Maria-Gabriella; Giancola, Guerino. Understanding Ultra Wide Band Radio Fundamentals, 1st Edition, (c) N/A. Reprinted by permission of Pearson Education, Inc., Upper Saddle River, NJ.

[20] Anuj Batra, Jaiganesh Balakrishnan, G. Roberto Aiello, Jeffrey R. Foerster. Design of a Multiband OFDM System for Realistic UWB Channel Environments. IEEE Transactions on Microwave Theory and Techniques, 52(9), Sept. 2004.

[21] S. S. Ghassemzadeh, V. Tarokh. UWB Path Loss Characterization in Residential Environments. In IEEE Radio Frequency Integrated Circuits (RFIC) Symposium, pages $501-504$, June 2003 .

[22] M. Z. Win, R. A. Scholtz. On the Robustness of Ultra-wide Bandwidth Signals in Dense Multipath Environments. IEEE Communications Letters, Volume 2, Issue 2:51 - 53, Feb. 1998.

[23] R. Price, P. E. Green Jr. A Communication Technique for Multipath Channels. In Proc. IRE, volume 46, pages 555 - 570, March 1958.

[24] D. Cassioli, M. Z. Win, F. Vatalaro, A. F. Molisch. Performance of Low-Complexity Rake Reception in a Realistic UWB Channel. IEEE International Conference on Communications, volume 2:763 - 767, May 2002.

[25] M. Z. Win, R. A. Scholtz. Characterization of Ultra-Wide Bandwidth Wireless Indoor Channels: A Communication-Theoretic View. IEEE Journal on Selected Areas in Communications, 20, Issue 9:1613 - 1627, Dec. 2002.

[26] M. Jeruchim. Techniques for Estimating the Bit Error Rate in the Simulation of Digital Communication Systems. IEEE Journal on Selected Areas in Communications, volume 2:153 - 170, Jan. 1984.

[27] Nazli Güney, Hakan Deliç, Mutlu Koca. Robust Rake Receiver for UWB Impulse Radio. In IEEE International Conference on Ultra-Wideband, Switzerland (submitted), Sept. 2005. 
[28] D. Cassioli, M. Z. Win, F. Vatalaro, A. F. Molisch. Effects of Spreading Bandwidth on the Performance of UWB RAKE Receivers. IEEE International Conference on Communications, volume 5:3545 - 3549, May 2003.

[29] Mohammad Azizur Rahman, Shigenobu Sasaki, Jie Zhou, Shogo Muramatsu, Hisakazu Kikuchi. Performance Evaluation of RAKE Reception of Ultra Wideband Signals over Multipath Channels from Energy Capture Perspective. In International Workshop on Joint UWBST \& IWUWBS, pages 231 - 235, May 2004.

[30] F. E. Aranda, N. Brown, H. Arslan. Rake Receiver Finger Assignment For Ultrawideband Radio. Advances in Wireless Communications Signal Processing, pages 239 - 243, June 2003.

[31] D. R. McKinstry, R. M. Buehrer. UWB Small Scale Channel Modeling and System Performance. In Vehicular Technology Conference VTC 2003-Fall, volume 1, pages $6-10$, Oct. 2003.

[32] D. Cassioli, M. Z. Win, A. F. Molisch. The Ultra-Wide Bandwidth Indoor Channel: from Statistical Model to Simulations. IEEE Journal on Selected Areas in Communications, volume 20:1247 - 1257, Aug. 2002.

[33] G. D. Weeks, J. K. Townsend, J. A. Freebersyser. Performance of Hard Decision Detection for Impulse Radio. In Proc. IEEE MILCOM '99, Oct. 1999.

[34] B. Mielczarek, M. O. Wessman, A. Svensson. Performance of Coherent UWB Rake Receivers with Channel Estimators. In IEEE Vehicular Technology Conference, volume 3, pages 1880 - 1884, Oct. 2003.

[35] Huaping Liu. Error Performance of a Pulse Amplitude and Position Modulated Ultrawideband System over Lognormal Fading Channels. IEEE Communications Letters, 7(11):531 - 533, Nov. 2003.

[36] R. Tesi, M. Hamalainen, J. Inatti, J. Oppermann, V. Hovinen. On the Multi-User Interference Study for Ultra Wideband Communication Systems in AWGN and Mod- 
ified Saleh-Valenzuela Channel. In International Workshop on Joint UWBST \& IWUWBS, pages 91 - 95, May 2004.

[37] S. M. Yano. Investigating the Ultra-Wideband Indoor Wireless Channel. In Vehicular Technology Conference, volume 3, pages 1200 - 1204, May 2002.

[38] Yannis Viniotis. Probability and Random Processes for Electrical Engineers. WCB McGraw-Hill, 1998. 